Winterthurerstr. 190

CH-8057 Zurich

http://www.zora.uzh.ch

Year: 2009

\title{
Search for the rare decays $\mathrm{B}+-->\mathrm{K}+-\mathrm{mu}+$ mu- and $\mathrm{B} 0 \mathrm{~d}->\mathrm{K}^{*}$ $\mathrm{mu}+$ mu- with the D0 Experiment
}

\author{
Wenger, A
}

Wenger, A. Search for the rare decays B+- -\&gt; K+- mu+ mu- and B0d -\&gt; K* mu+ mu- with the D0

Experiment. 2009, University of Zurich, Faculty of Science.

Postprint available at:

http://www.zora.uzh.ch

Posted at the Zurich Open Repository and Archive, University of Zurich.

http://www.zora.uzh.ch

Originally published at:

University of Zurich, Faculty of Science, 2009. 


\title{
Search for the rare decays $\mathrm{B}+-->\mathrm{K}+-\mathrm{mu}+$ mu- and $\mathrm{B} 0 \mathrm{~d}->\mathrm{K}^{*}$ $\mathrm{mu}+$ mu- with the D0 Experiment
}

\begin{abstract}
The study of processes involving flavour-changing neutral currents provides a particularly promising probe for New Physics beyond the Standard Model of particle physics. These processes are forbidden at tree level and proceed through loop processes, which are strongly suppressed in the Standard Model. Cross-sections for these processes can be significantly enhanced by contributions from new particles as they are proposed in most extentions of the Standard Model. This thesis presents searches for two flavour-changing neutral current decays, $\mathrm{B} \pm$ ! $\mathrm{K} \pm \mu+\mu-$ and $\mathrm{B} 0 \mathrm{~d}$ ! $\mathrm{K} \propto \mu+\mu-$. The analysis was performed on $4.1 \mathrm{fb}-1$ of data collected by the DØ detector in Run II of the Fermilab Tevatron. Candidate events for the decay $\mathrm{B} \pm ! \mathrm{K} \pm \mu+\mu-$ were selected using a multi-variate analysis technique and the number of signal events determined by a fit to the invariant mass spectrum. Normalising to the known branching fraction for $\mathrm{B} \pm$ ! J/Ã \pm , a branching fraction of $\mathrm{B}\left(\mathrm{B} \pm ! \mathrm{K} \pm \mu^{+} \mu^{-}\right)=6.45 \pm 2.24$ (stat) \pm 1.19 (syst) $\times$ 10-7 (1) was measured. The branching fraction for the decay B0 d ! Ka $\mu+\mu-$ was determined in a similar way. Normalizing to the known branching fraction for B0 d! J/ÃKa, a branching fraction of $\mathrm{B}\left(\mathrm{B} 0 \mathrm{~d} ! \mathrm{K} \propto \mu^{+} \mu^{-}\right)=11.15 \pm 3.05$ (stat) \pm 1.94 (syst) $\times 10^{-7}$ (2) was measured. All measurements are in agreement with the Standard Model.
\end{abstract}




\section{Search for the Rare Decays $B^{ \pm} \rightarrow K^{ \pm} \mu^{+} \mu^{-}$ and $B_{d}^{0} \rightarrow K^{*} \mu^{+} \mu^{-}$with the $\mathbf{D} \emptyset$ Experiment}

\section{Dissertation}

zur

Erlangung der naturwissenschaftlichen Doktorwürde

(Dr. sc. nat.)

vorgelegt der

Mathematisch-naturwissenschaftlichen Fakultät

der

Universität Zürich

von

Andreas Wenger

aus

Bellwald

Promotionskomitee

Prof. Dr. Ulrich Straumann (Vorsitz)

PD Dr. Frank Lehner

Zürich 2009 

In Loving Memory,

Werner Wenger

- 30 1 th June 1989

Annemarie Wenger

- $18^{\text {th }}$ May 2008 



\begin{abstract}
The study of processes involving flavour-changing neutral currents provides a particularly promising probe for New Physics beyond the Standard Model of particle physics. These processes are forbidden at tree level and proceed through loop processes, which are strongly suppressed in the Standard Model. Cross-sections for these processes can be significantly enhanced by contributions from new particles as they are proposed in most extentions of the Standard Model.

This thesis presents searches for two flavour-changing neutral current decays, $B^{ \pm} \rightarrow$ $K^{ \pm} \mu^{+} \mu^{-}$and $B_{d}^{0} \rightarrow K^{*} \mu^{+} \mu^{-}$. The analysis was performed on $4.1 \mathrm{fb}^{-1}$ of data collected by the DØ detector in Run II of the Fermilab Tevatron.

Candidate events for the decay $B^{ \pm} \rightarrow K^{ \pm} \mu^{+} \mu^{-}$were selected using a multi-variate analysis technique and the number of signal events determined by a fit to the invariant mass spectrum. Normalising to the known branching fraction for $B^{ \pm} \rightarrow J / \psi K^{ \pm}$, a branching fraction of

$$
\mathcal{B}\left(B^{ \pm} \rightarrow K^{ \pm} \mu^{+} \mu^{-}\right)=6.45 \pm 2.24(\text { stat }) \pm 1.19 \text { (syst) } \times 10^{-7}
$$

was measured.

The branching fraction for the decay $B_{d}^{0} \rightarrow K^{*} \mu^{+} \mu^{-}$was determined in a similar way. Normalizing to the known branching fraction for $B_{d}^{0} \rightarrow J / \psi K^{*}$, a branching fraction of

$$
\mathcal{B}\left(B_{d}^{0} \rightarrow K^{*} \mu^{+} \mu^{-}\right)=11.15 \pm 3.05(\text { stat }) \pm 1.94(\text { syst }) \times 10^{-7}
$$

was measured.
\end{abstract}

All measurements are in agreement with the Standard Model. 


\section{Zusammenfassung}

Flavour-ändernde neutrale Ströme sind im Standardmodell durch ihre "loop"-Struktur stark unterdrückt und sind daher gut geeignet nach neuer Physik zu suchen. Die Existenz neuer Teilchen würde den Zerfall durch ihre "loop"-Struktur beeinflussen. Diese Arbeit präsentiert die Suche nach den Flavour-ändernden neutralen Strömen in den Zerfällen $B^{ \pm} \rightarrow K^{ \pm} \mu^{+} \mu^{-}$und $B_{d}^{0} \rightarrow K^{*} \mu^{+} \mu^{-}$. Die Analyse basiert auf Daten $\left(4.1 \mathrm{fb}^{-1}\right)$ welche mit dem DØ-Detektor im Run II des Tevatron-Beschleunigers am Fermilab aufgezeichnet wurden.

Die Zerfallsamplitude von $B^{ \pm} \rightarrow K^{ \pm} \mu^{+} \mu^{-}$wird mit Hilfe des Normierungskanals $B^{ \pm} \rightarrow J / \psi K^{ \pm}$bestimmt, damit sich einige systematischen Fehler auf die Messung herauskürzen. Durch das Bestimmen der Signal Events ueber dem Untergrund kann das Verzweigungsverhältnis bestimmt werden. Es wurde ein Verzweigungsverhältnis von

$$
\mathcal{B}\left(B^{ \pm} \rightarrow K^{ \pm} \mu^{+} \mu^{-}\right)=6.45 \pm 2.24(\text { stat }) \pm 1.19(\text { syst }) \times 10^{-7}
$$

gefunden.

Beim Zerfall $B_{d}^{0} \rightarrow K^{*} \mu^{+} \mu^{-}$wurde das Verzweigungsverhältnis $\mathcal{B}\left(B_{d}^{0} \rightarrow K^{*} \mu^{+} \mu^{-}\right)$ bestimmt, indem die beobachtete Anzahl von Ereignissen in der $B_{d}^{0}$ Signalregion auf die Anzahl der rekonstruierten $B_{d}^{0} \rightarrow J / \psi K^{*}(892)$ Ereignisse normiert wird. Es wurde das Verzweigungsverhältnis von

$$
\mathcal{B}\left(B_{d}^{0} \rightarrow K^{*} \mu^{+} \mu^{-}\right)=11.15 \pm 3.05(\text { stat }) \pm 1.94(\text { syst }) \times 10^{-7}
$$

ermittelt.

Alle Messungen stimmen mit den Standard Modell Vorhersagen ueberein. 


\section{Contents}

Introduction 1

1 Standard Model 3

1.1 Fermions - Quarks and Leptons . . . . . . . . . . . . . . . . . . 3

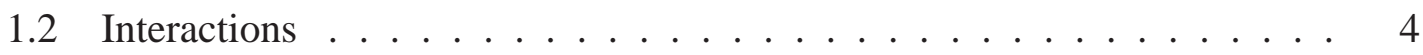

1.3 The CABIBBO-KOBAYASHI-MASKAWA Matrix and $B$ physics . . . 5

1.3.1 Rare decays of B mesons . . . . . . . . . . . . . . . 7

1.4 The Future of the Standard Model . . . . . . . . . . . . . . . . . . . 8

2 Flavour changing neutral current 9

2.1 FCNC and the GIM Mechanism . . . . . . . . . . . . . . . . 9

2.2 Box and Penguin Diagrams . . . . . . . . . . . . . . . . 10

2.3 Effective Hamiltonian and Operator Product Expansion . . . . . . . . . . 10

$2.4 \quad B \rightarrow X_{s} \gamma \ldots \ldots \ldots \ldots \ldots \ldots \ldots$

$2.5 B \rightarrow X_{s} \ell^{+} \ell^{-} \ldots \ldots \ldots \ldots \ldots \ldots$

$2.5 .1 \quad$ Exclusive decays . . . . . . . . . . . . . . . 17

2.6 Normalisation processes . . . . . . . . . . . . . . . . . 18

3 The DØ experiment at the Tevatron 21

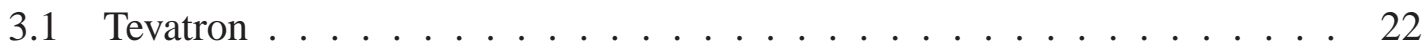

3.1.1 Production of protons and pre-acceleration . . . . . . . . 23

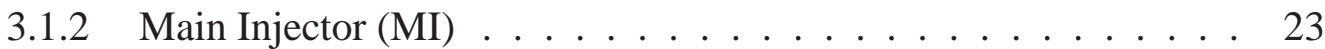

3.1.3 The Tevatron Ring Synchrotron _. . . . . . . . . . . . . 23

3.2 The $\mathrm{D} \emptyset$ Detector . . . . . . . . . . . . . . . . . . . 24 
3.2.1 The coordinate System . . . . . . . . . . . . . . 26

3.2.2 Central Tracking System . . . . . . . . . . . . . . 26

3.2 .3 Calorimeter system . . . . . . . . . . . . . . 33

3.2 .4 Muon System . . . . . . . . . . . . . . . . . . . . 34

3.2 .5 Luminosity System . . . . . . . . . . . . . . . . . 36

3.2 .6 Trigger System . . . . . . . . . . . . . . . . . 37

4 Event Reconstruction $\quad 39$

4.1 Track Reconstruction . . . . . . . . . . . . . . . . . . . . . 40

4.1.1 HFT Tracking Algorithm . . . . . . . . . . . . . . . 40

4.1 .2 AA algorithm . . . . . . . . . . . . . . . 40

4.2 Muons . . . . . . . . . . . . . . . . . . . . . 41

4.3 Vertex Reconstruction . . . . . . . . . . . . . . . . . . . . 42

5 Stability checks of the tracking alignment 45

5.1 Cosmics Data, reconstruction and geometry . . . . . . . . . . 46

5.2 Alignment procedure . . . . . . . . . . . . . . . . 46

5.3 Different geometry modifications . . . . . . . . . . . . . . . 47

5.3.1 Shift of SMT layer 3 and layer 6 - random shifts . . . . . . . . 48

5.4 Summary and Conclusion . . . . . . . . . . . . . . . . . . 51

6 Multivariate Analysis Method 53

6.1 Decision tree . . . . . . . . . . . . . . . . 53

6.2 Boosted Decision Tree . . . . . . . . . . . . . . . . 55

6.2.1 AdaBoost-Algorithm . . . . . . . . . . . . . 55

6.3 Toolkit for Multivariate Analysis . . . . . . . . . . . . . . . 56

6.4 Optimization . . . . . . . . . . . . . . . . 56

7 Data and Event Selection $\quad 61$

7.1 Data Sample and Selection . . . . . . . . . . . . . . . 61

$7.2 \quad B$-physics trigger strategy . . . . . . . . . . . . . . 61

7.2.1 Muon Triggers . . . . . . . . . . . . . . . . . 62

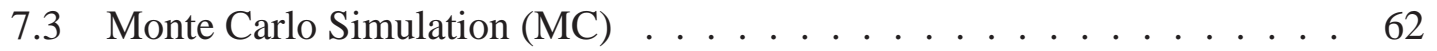

7.4 Discriminating Variables . . . . . . . . . . . . . . . . 65 
$8 \quad$ Rare Decay $B^{ \pm} \rightarrow K^{ \pm} \mu^{+} \mu^{-}$

8.1 Introduction . . . . . . . . . . . . . . . . . . . 69

8.2 Pre-selection ........................ 71

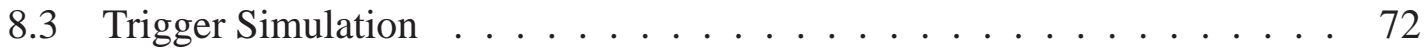

8.4 Final selection - TMVA . . . . . . . . . . . . . . . . . 77

8.5 Results . . . . . . . . . . . . . . . . . . . 82

8.5.1 Branching fraction for $B^{ \pm} \rightarrow K^{ \pm} \mu^{+} \mu^{-} \ldots \ldots . \ldots 82$

9 The decay $B_{d}^{0} \rightarrow K^{*} \mu^{+} \mu^{-} \quad 87$

9.1 Introduction . . . . . . . . . . . . . . . . 87

9.2 Pre-selection . . . . . . . . . . . . . . . 88

9.3 Final selection - TMVA . . . . . . . . . . . . . . . . 90

9.4 Results . . . . . . . . . . . . . . . . . . . 93

10 Conclusion $\quad 99$

$\begin{array}{ll}\text { Bibliography } & 101\end{array}$

$\begin{array}{ll}\text { Acknowledgments } & 108\end{array}$

$\begin{array}{lr}\text { Curriculum Vitae } & 110\end{array}$ 


\section{Introduction}

Particle physics is the branch of the physical sciences that aims to understand the elementary constituents of the universe and the laws which govern their interactions. The Human race always wondered about what makes up the world around them. The idea that the world was composed of small indivisible units of matter began with Democritus, a Greek philosopher in the 5th century BC. Around 1800 it was discovered that the world is made of molecules and that molecules are made of atoms. For all the efforts of early scientists, the investigation of fundamental particles as we know them today did not begin in earnest until 1897, with the discovery of the electron by J.J. Thomson. Its discovery paved the way for further explorations of the sub-atomic world. By now a whole particle "Zoo" has been discovered but physicist still can only describe a small part of the universe.

The theories of particle physics, collectively known as the "Standard Model", have withstood testing against mountains of data during the past fifty years with only minor modifications. Yet the theory is incomplete: there is no universally accepted and experimentally tested extension that would combine the Standard Model with the other grand theory of physics, Albert Einstein's General Relativity, the theory of gravity. The universally accepted model of cosmology asserts that the universe is composed of $74 \%$ "dark energy" and $22 \%$ "cold dark matter". Neither of these two constituents are described by or known to the Standard Model. A mere $4 \%$ of the universe is visible in stars, galaxies and gas clouds and is described by the Standard Model. The Standard Model still lacks the experimental observation of the last key element, the Higgs boson, which is the quantum of the scalar field that creates the mass of the elementary particles.

The most important method for testing the Standard Model is accelerating particles to high energies and colliding them. An important parameter of an accelerator is the energy of the colliding particles. Currently the accelerator "Tevatron" close to Chicago produces the highest energy at collisions. Soon the Large Hadron Collider (LHC) at CERN in Geneva will start and increases the energy by a factor of 7. The LHC will provide new possibilities to probe the Standard Model and looks for physics beyond the Standard Model.

This thesis presents measurements, using data collected by the DØ experiment at the Tevatron between 2001 and 2008, of the branching ratios of two flavour changing neutral 
current B hadron decays: $B_{u}^{ \pm} \rightarrow K^{ \pm} \mu^{+} \mu^{-}$and $B_{d}^{0} \rightarrow K^{\star} \mu^{+} \mu^{-}$. The layout of the thesis is as follows. The first Chapter provides a brief introduction to the Standard Model followed by some theoretical background on FCNC decays of B mesons. In Chapter 3 a brief description is given of the Tevatron accelerator and the $\mathrm{D} \varnothing$ detector. For precise measurements the detector has to be spatially aligned and some alignment studies are presented in Chapter 4. Chapter 5 provides a brief introduction to event reconstruction and object identification at $\mathrm{D} \varnothing$ while the data and event selection is presented in Chapter 6. The methods used for the analysis and the optimisation are described in Chapter 7. Following this, Chapters 8 and 9 present the analysis of the particle decays $B_{u}^{ \pm} \rightarrow K^{ \pm} \mu^{+} \mu^{-}$ and $B_{d}^{0} \rightarrow K^{\star} \mu^{+} \mu^{-}$. The thesis is concluded with an outlook and a summary. 


\section{Chapter 1}

\section{Standard Model}

The Glashow-Salam-Weinberg (GSW) model [1, 2, 3], which unifies the electromagnetic and weak interactions into the electroweak theory and the so called Quantum Chromodynamics (QCD) [4], which explains the strong interactions between the particles, form the Standard Model (SM) of particle physics [5]. The fourth fundamental force of nature, gravity, which is perhaps the most important for all the macroscopic phenomena, is not included in the theoretical framework of the SM. According to the SM, there are two classes of fundamental particles that shape our universe, the spin $1 / 2$ fermions which are the matter particles and the spin 1 gauge bosons, which are the force carriers between the fermions.

\subsection{Fermions - Quarks and Leptons}

The fermions are further classified into leptons (l) and quarks (q). There are six flavours of leptons: the electron $(e)$, the muon $(\mu)$, the tau $(\tau)$ and their corresponding neutrinos $\left(\nu_{e}, \nu_{\mu}, \nu_{\tau}\right)$. The electric charged leptons interact via the electromagnetic and weak forces, while the neutrinos, which carry no electric charge interact only via the weak force. In the SM the neutrinos were presumed to be massless, but experimentally their masses have been constraint to be non-zero but small [6] (see Table 1.1). There are also six flavours of quarks: up $(u)$, down $(d)$, charm $(c)$, strange $(s)$, top $(t)$ and bottom $(b)$. Quarks interact via the strong force as well as the electromagnetic and weak forces. The strong interaction binds the quarks into a spectrum of particles called hadrons. The fermions can be classified in three generations according to their rising masses as presented in Table 1.2 and 1.1. The SM does not predict the number of generations. 
Table 1.1: The three generations of the Leptons.

\begin{tabular}{lcccc}
\hline \hline \multicolumn{5}{c}{ Leptons } \\
\hline \hline Particle Type & Symbol & Charge & Mass (MeV) & Generation \\
\hline electron neutrino & $\nu_{e}$ & 0 & $<0.000003$ & 1 \\
electron & $e$ & -1 & 0.511 & \\
\hline muon neutrino & $\nu_{\mu}$ & 0 & $<0.19$ & 2 \\
muon & $\mu$ & -1 & 105.6 & \\
\hline tau neutrino & $\nu_{\tau}$ & 0 & $<18.2$ & 3 \\
tau & $\tau$ & -1 & 1777 & \\
\hline \hline
\end{tabular}

Table 1.2: The fundamental quarks.

\begin{tabular}{lcccc}
\hline \hline \multicolumn{5}{c}{ Quarks } \\
\hline \hline Particle Type & Symbol & Charge & Mass (GeV) & Generation \\
\hline up quark & $u$ & $2 / 3$ & 0.003 & 1 \\
down quark & $d$ & $-1 / 3$ & 0.005 & \\
\hline charm quark & $c$ & $2 / 3$ & 1.2 & 2 \\
strange quark & $s$ & $-1 / 3$ & 0.1 & \\
\hline top quark & $t$ & $2 / 3$ & 178 & \multirow{2}{*}{3} \\
bottom quark & $b$ & $-1 / 3$ & 4.5 & \\
\hline \hline
\end{tabular}

\subsection{Interactions}

There are four fundamental forces (see Table 1.3) that lead to interactions between the matter particles: electromagnetic, weak, strong and gravitation. The electromagnetic, weak and strong forces can be expressed as a quantum field theories, where the interactions are mediated by a boson exchange between the interacting particles.

- Electromagnetic Force The quantum field theory of the electrodynamic force is the Quantum Electrodynamics or QED [7]. This theory explains the interactions of all particles due to the electric charge by the exchange of photons. Since the photons are massless the range of the force is infinite. 
- Weak Force The weak force is responsible for particle decays that change the type of particle, such as quark flavours or lepton types. Due to the massive force carriers, $\mathrm{W}$ and $\mathrm{Z}$ bosons, the weak force has a short range.

- Strong force The strong force binds quarks into hadrons. Strong interactions are mediated by the exchange of gluons which carry colour charge and are described by the Quantum Chromodynamics or QCD. The "Short range" refers to the fact that the force dies off exponentially in distance. This means that a nucleon is only affected by the strong force of its nearest neighbors.

- Gravitational Force Gravity, which is classically explained by Einstein's General Relativity is responsible for interactions between massive bodies. Until now there does not exist a correct formulation for a quantum theory of gravity that would explain the interactions as the exchange of a graviton boson. In particle physics gravity is generally disregarded due to the low masses of the elementary particles, however its effects may have some undiscovered influences.

Table 1.3: The fundamental forces and properties.

\begin{tabular}{lcccccc}
\hline \hline \multicolumn{7}{c}{ Bosons } \\
\hline \hline Force & Carrier & Range $(\mathrm{cm})$ & Relative Strength & Mass $(\mathrm{GeV})$ & Charge & Spin \\
\hline \multirow{3}{*}{ Weak } & $W^{+}$ & & & 80.4 & +1 & 1 \\
& $W^{-}$ & $10^{-16}$ & $10^{-6}$ & 80.4 & -1 & 1 \\
& $Z$ & & & 91.2 & 0 & 1 \\
\hline EM & photon $(\gamma)$ & infinite & $10^{-2}$ & 0 & 0 & 1 \\
\hline Strong & gluon $(\mathrm{g})$ & $\sim 10^{-13}$ & 1 & 0 & 0 & 1 \\
\hline \hline
\end{tabular}

\subsection{The CABIBBO-KOBAYASHI-MASKAWA Matrix and $B$ physics}

The exploration of physics with $b$ flavoured hadrons ( $B$ mesons) offers a very good testing ground for the SM description of the electroweak interactions. $C P$ is the combined transformation of charge conjugation $C$ and the parity transformation $P$. The parity transformation is an inversion of all space coordinates and the charge conjugation reverts the sign of all charge-like quantum numbers from a particle. The $\mathrm{CP}$ violation, which was 
discovered in 1964 by Christenson, Cronin, Fitch and Turlay in the neutral kaon system [8], is still one of the experimentally least constrained phenomena. The other main topic is the study of rare $b$ decays induced by flavour changing neutral current (FCNC) transitions $b \rightarrow s, d$ which are loop-suppressed in the SM and thus very sensitive to new physics.

The $B$ system appears to be most promising for testing the $\mathrm{CP}$ violation in the SM in a quantitative way $[9,10,11]$. The CP violation in the SM is closely related to the Cabibbo-Kobayashi-Maskawa (CKM) matrix $[12,13]$. For for the discovery of the origin of the broken symmetry which predicts the existence of at least three families of quarks in nature Kobayashi and Maskawa got the Noble Prize in 2008. This matrix connects the electroweak eigenstates $\left(d^{\prime}, s^{\prime}, b^{\prime}\right)$ of the down, strange and bottom quarks with their mass eigenstates $(d, s, b)$ through the following unitary transformation:

$$
\left(\begin{array}{c}
d^{\prime} \\
s^{\prime} \\
b^{\prime}
\end{array}\right)=\left(\begin{array}{lll}
V_{u d} & V_{u s} & V_{u b} \\
V_{c d} & V_{c s} & V_{c b} \\
V_{t d} & V_{t s} & V_{t b}
\end{array}\right) \cdot\left(\begin{array}{c}
d \\
s \\
b
\end{array}\right)=V(C K M) \cdot\left(\begin{array}{c}
d \\
s \\
b
\end{array}\right)
$$

The CKM matrix is a $3 \times 3$ unitary matrix with $n^{2}$ free parameters to describe the matrix. The phases are arbitrary, so $2 n-1$ parameters can be eliminated by phase rotations and leading to $(n-1)^{2}$ independent parameters. In case of three generations $(n=3)$, the CKM matrix contains four independent parameters which are represented by three Euler angles and a single complex phase. The three rotation angles are referred as mixing angles and the complex phase allows for $\mathrm{CP}$ violation.

The values of the individual matrix elements can in principle all be determined from weak decays of the relevant quarks or in some cases from deep inelastic neutrino scattering. Table 1.4 shows the strength and a possible method to measure the different CKM matrix elements and it can be seen that the diagonal elements are highly favored and those elements farthest from the diagonal are the most suppressed. Concerning test of the $\mathrm{CP}$ violation the central targets are probing the unitarity relations of the CKM matrix. The unitarity of the CKM matrix is described by

$$
V_{C K M}^{\dagger} \cdot V_{C K M}=1
$$

This leads to six relations which can be described by triangles in the complex plane which all have the same area [15]. In only two of this six triangles the three sides are of comparable magnitude:

$$
\begin{aligned}
& V_{u d} V_{u b}^{*}+V_{c d} V_{c b}^{*}+V_{t d} V_{t b}^{*}=0 \\
& V_{u d}^{*} V_{t d}+V_{u s}^{*} V_{t s}+V_{u b}^{*} V_{t b}=0
\end{aligned}
$$

The triangle described by the first Equation in 1.3 is presented in Fig. 1.1.

The unitarity triangle provides an important test of the CKM mechanism. The sides and angles of the triangle can be measured and should "close" within the confines of the SM. If the triangle does not close, it would be an indication of New Physics beyond the SM. 
Table 1.4: Quark transitions and their strength [14].

\begin{tabular}{lcc}
\hline \hline Quark Transition & Strength & Method of Measurement \\
\hline$V_{u d}$ & $0.97397-0.97441$ & Nuclear $\beta$ decay \\
$V_{u s}$ & $0.2247-0.2267$ & $\bar{K}^{0} \rightarrow \pi^{+} e^{-} \bar{\nu}_{e}$ \\
$V_{u b}$ & $0.00343-0.00375$ & $B \rightarrow \pi l \bar{\nu}_{l}$ \\
$V_{c d}$ & $0.2246-0.2266$ & $D^{0} \rightarrow \pi^{+} e^{-} \bar{\nu}_{e}$ \\
$V_{c s}$ & $0.97311-0.97357$ & $D^{0} \rightarrow K^{-} e^{+} \nu_{e}$ \\
$V_{c b}$ & $0.0404-0.0425$ & $B \rightarrow \chi_{c} l \bar{\nu}_{l}$ \\
$V_{t d}$ & $0.00837-0.00900$ & $B-B^{0}$ mixing \\
$V_{t s}$ & $0.0397-0.0417$ & $b \rightarrow s$ \\
$V_{t b}$ & $0.999090-0.999177$ & $t \rightarrow b W$ \\
\hline \hline
\end{tabular}

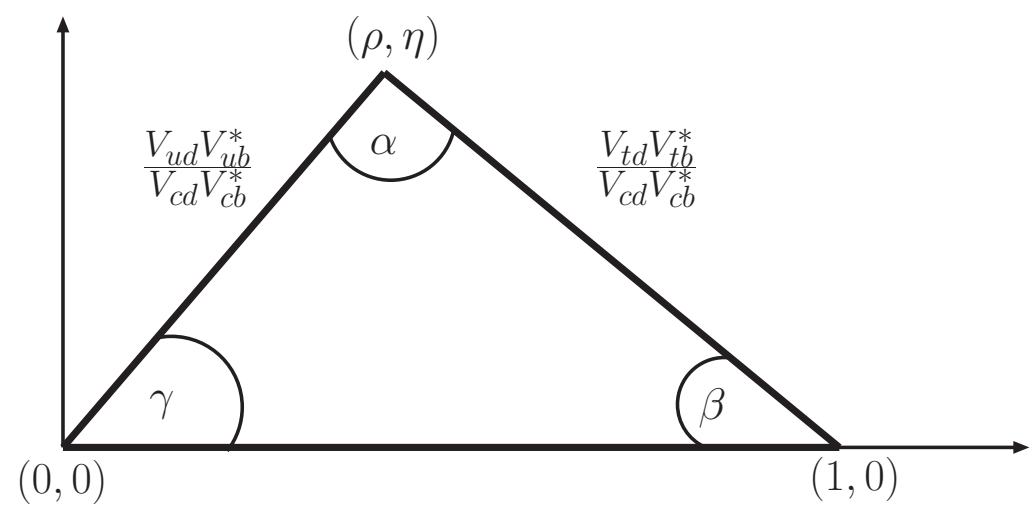

Figure 1.1: The unitarity triangle.

\subsubsection{Rare decays of $B$ mesons}

In the common understanding, rare B decays are due to heavily Cabibbo-suppressed $b \rightarrow$ $u$ transitions or flavour-changing neutral currents (FCNC) $b \rightarrow s$ or $b \rightarrow d$ which are forbidden in the SM at tree-level. They proceed at higher order in loop induced effects. In Table 1.4 it can be seen, that the strength of the matrix element $V_{t s}$ is small. The FCNC will be discussed in more detail in section 2 . 


\subsection{The Future of the Standard Model}

To date, the SM fits with all experimental evidence but it is not complete. It contains the Higgs mechanism to give particles physical masses, and requires the predicted Higgs boson, which is yet undiscovered. By calculating the mass of the Higgs in the SM including radiative corrections the mass diverges quadratically and to solve this problem New Physics is expected at the TeV scale [16]. There are other unsolved problems and questions in the SM such as why the top quark is so much heavier than the other quarks or why we have only three generations and how to include gravity. For many years, theorists have been working on trying to unify the forces; that is, to show that all four forces of nature can be derived from a single force. The SM coupling strengths extrapolated to very high energies do not converge at a single point. However, the introduction of Physics beyond the SM such as Supersymmetry (SUSY) causes the coupling strengths to converge at a single point (see Fig. 1.2). This new induced heavy particles can appear in loops and can enhance the branching fraction of rare $B$ and FCNC decays significantly. Answers to some of these questions are expected to be found at Tevatron and the Large Hadron Collider (LHC) in Geneva.

\section{Standard Model}

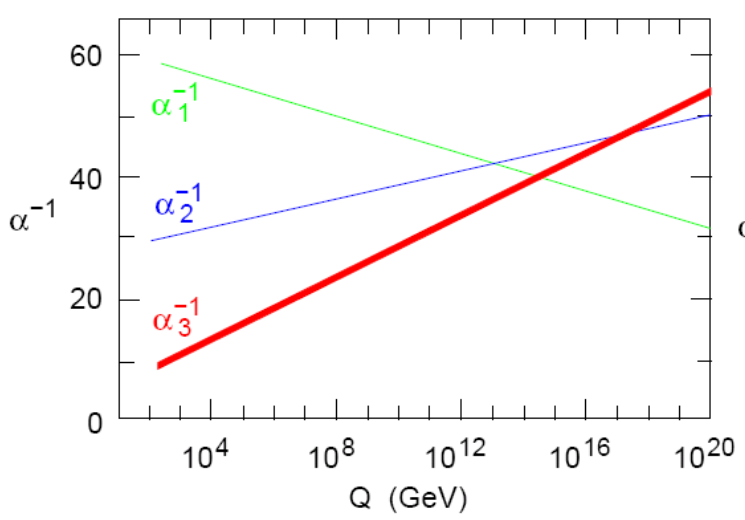

MSSM

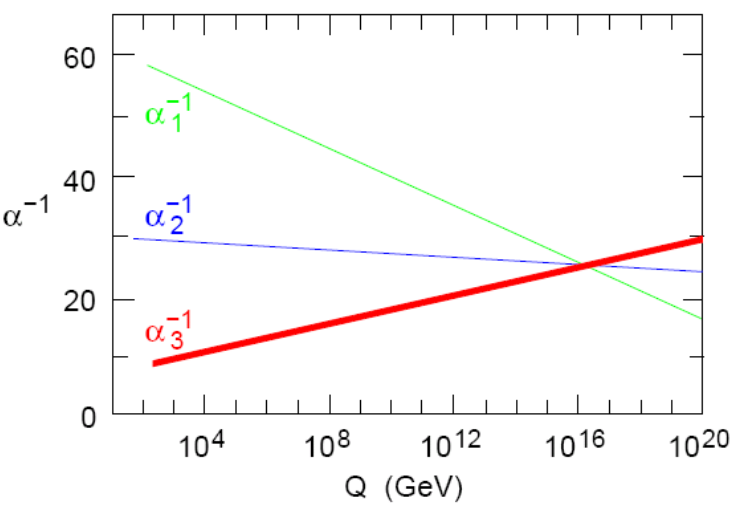

Figure 1.2: Unification of couplings constants $\left(a_{i}=g_{i}^{2} /(4 \pi)\right)$ in the minimal supersymmetric model (MSSM) as compared to failure without supersymmetry [16]. ( $a_{1}=$ electromagnetic, $a_{2}=$ weak and $a_{3}=$ strong coupling constant) 


\section{Chapter 2}

\section{Flavour changing neutral current}

\subsection{FCNC and the GIM Mechanism}

FCNC are well suited to probe the SM. The FCNC transitions $b \rightarrow s$ or $b \rightarrow d$ are Cabibbo-suppressed and are forbidden in the SM at tree-level. In the SM the neutral currents (NC) are flavour conserving and there is no direct coupling between the $b$ and the $s$ or $d$ quark. The GIM mechanism (Glashow-Iliopoulos-Maiani [17]) explains the non-existence of FCNC at tree level in the SM. For a two family quark model with the mixing angle $\theta_{C}$ the CKM matrix can be written as

$$
\left(\begin{array}{c}
d^{\prime} \\
s^{\prime}
\end{array}\right)=\left(\begin{array}{cc}
\cos \theta_{C} & \sin \theta_{C} \\
-\sin \theta_{C} & \cos \theta_{C}
\end{array}\right) \cdot\left(\begin{array}{c}
d \\
s
\end{array}\right)
$$

The contributions to the $\mathrm{NC}$ at tree level can then be summarized by four fundamental contributions:

$$
N C=u \bar{u}+d^{\prime} \bar{d}^{\prime}+s^{\prime} \bar{s}^{\prime}+c \bar{c}
$$

With Equation 2.1:

$$
\begin{gathered}
N C=u \bar{u}+d \bar{d} \cdot \cos ^{2} \theta_{C}+s \bar{s} \cdot \sin ^{2} \theta_{C}+(d \bar{s}+s \bar{d}) \cdot \cos \theta_{C} \cdot \sin \theta_{C} \\
+d \bar{d} \cdot \sin ^{2} \theta_{C}+s \bar{s} \cdot \cos ^{2} \theta_{C}-(d \bar{s}+s \bar{d}) \cdot \cos \theta_{C} \cdot \sin \theta_{C}+c \bar{c}
\end{gathered}
$$

The flavour changing amplitude $(d \bar{s}+s \bar{d})$ in Equation 2.3 cancel out so that finally the $\mathrm{NC}$ consists of the sum of the $q \bar{q}$ pairs.

$$
N C=u \bar{u}+d \bar{d}+s \bar{s}+c \bar{c}
$$

However, FCNC contributions to such processes are possible through so-called box and penguin diagrams. These contributions make it possible to observe FCNC. FCNC processes are probing virtual particles in the loop and can thus discover new physics. 


\subsection{Box and Penguin Diagrams}

As discussed in the previous section, the FCNC is absent in the SM at tree level but can occur at one loop level. Some possible box and penguin diagrams are shown in Fig. 2.1
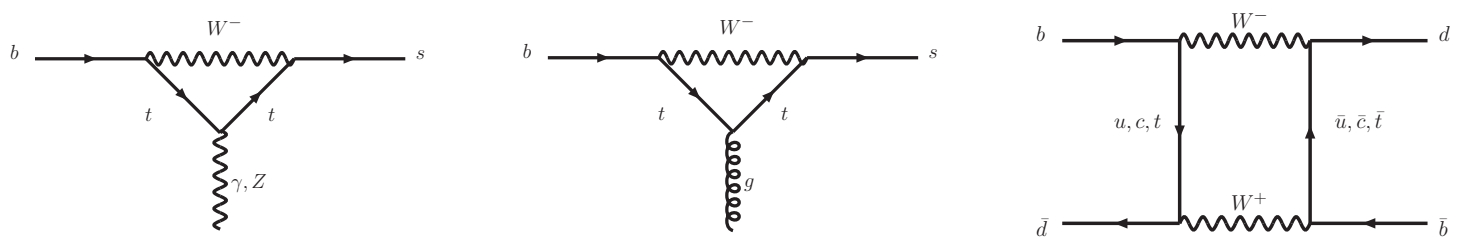

Figure 2.1: Example of penguin and box diagrams

The effective vertices can be calculated by using elementary vertices and propagators, and effective Feynman rules can be derived [18]. The suppression of these diagrams originates from the higher order in the gauge couplings. Their relative importance depends on the mass of the internal fermion lines, which explains the importance of the contribution of the top quark and the relevant CKM matrix element and also the sensitivity to heavy new particles.

In the box and penguin diagrams appears a loop factor $g^{2} / 16 \pi^{2} \sim 10^{-2}$ which suppresses its decay. Due to the mass difference $\delta m_{i}$ of the quarks involved in the loop additional (suppressing) factors of quadratic $\left(m_{i}^{2} / M_{W}^{2}\right)$ and logarithmic $\left(\log m_{i}^{2} / M_{W}^{2}\right)$ form appear. FCNC transitions for down-type (d, s and b) quarks are sensitive to the mass difference of the up-type (u,c and t) quarks in the loop and vice versa. On the basis of the large mass of the top quark $m_{t}>M_{W} \gg m_{c}, m_{u}$ the GIM mechanism is attenuated and the down-type FCNC processes $b \rightarrow s, d$ and $s \rightarrow d$ are enhanced.

\subsection{Effective Hamiltonian and Operator Product Expan- sion}

The amplitude for a decay of a given meson $M=K, B, \ldots$ into a final state $F$ can be described as:

$$
A(M \rightarrow F)=\left\langle F\left|\mathcal{H}_{e f f}\right| M\right\rangle
$$

where $\mathcal{H}_{\text {eff }}$ is the relevant Hamiltonian. These decays originate in weak transitions mediating a $W^{ \pm}$or a $Z$ boson. However, the presence of strong and electromagnetic interactions often has an important impact on weak decays. Due to the fact that the $W^{ \pm}$ and $Z$ are very massive the basic weak transition take place at very short distance scales $\mathcal{O}\left(1 / M_{W, Z}^{2}\right)$. The strong interactions take place at both short and long distances and generally weak decays of hadrons receive contributions from both. To separate short distance 
effects from long distance effects the Operator Product Expansion (OPE) [19] combined with the renormalization group is used. The OPE introduces local operators which are governing "effectively" the decay in question. All Hamiltonians considered can be written as linear combinations of local four-fermion operators and the final decay amplitude can be written as

$$
A(M \rightarrow F)=-\frac{4 G_{F}}{\sqrt{2}} \lambda_{C K M} \sum_{i} C_{i}(\mu)\left\langle F\left|Q_{i}(\mu)\right| M\right\rangle
$$

Here, $G_{F}$ is the Fermi constant, $\lambda_{C K M}$ is a CKM factor and $\mu$ is an appropriate renormalization scale. The local operators are denoted as $Q_{i}$, and $C_{i}$ stands for the Wilson coefficients, that are calculated by perturbative methods and are functions of the strong coupling constant $\alpha_{s}, M_{W}$ and $\mu$. The short distance contributions contained in $C_{i}(\mu)$ are separated by the scale $\mu$ from the long distance contributions contained in $\left\langle F\left|Q_{i}(\mu)\right| M\right\rangle$. For B decays the scale $\mu$ is chosen to be at the order of a few $\mathrm{GeV}$, i.e., around the mass of the $b$-quark. By evolving the scale from $\mu=Q\left(M_{W}\right)$ down to lower values of $\mu$ one transforms the physics information at scales higher than $\mu$ from the hadronic matrix element into $C_{i}(\mu)$. Since the full amplitude can not depend on the scale $\mu$, the $\mu$ dependence of the matrix element and the Wilson coefficients must cancel out. A set of basic operators entering the OPE can be specified at short distance. The typical diagrams in the full theory which these operators originate from are shown in Fig. 2.2. They can be classified in six classes [18]:

\section{Current-Current (Figure 2.2a):}

$$
\begin{aligned}
Q_{1} & =\left(\bar{s}_{L \beta} \gamma_{\mu} c_{L \alpha}\right)\left(\bar{c}_{L \alpha} \gamma^{\mu} b_{L \beta}\right) \\
Q_{2} & =\left(\bar{s}_{L} \gamma_{\mu} c_{L}\right)\left(\bar{c}_{L} \gamma^{\mu} b_{L}\right)
\end{aligned}
$$

\section{QCD-Penguins (Figure 2.2b):}

$$
\begin{aligned}
Q_{3} & =\left(\bar{s}_{L} \gamma_{\mu} b_{L}\right) \sum_{q=u, d, s, c, b}\left(\bar{q} \gamma^{\mu} q\right) \\
Q_{4} & =\left(\bar{s}_{L \alpha} \gamma_{\mu} b_{L \beta}\right) \sum_{q=u, d, s, c, b}\left(\bar{q}_{L \beta} \gamma^{\mu} q_{L \alpha}\right) \\
Q_{5} & =\left(\bar{s}_{L} \gamma_{\mu} b_{L}\right) \sum_{q=u, d, s, c, b}\left(\bar{q}_{R} \gamma^{\mu} q_{R}\right) \\
Q_{6} & =\left(\bar{s}_{L \alpha} \gamma_{\mu} b_{L \beta}\right) \sum_{q=u, d, s, c, b}\left(\bar{q}_{R \beta} \gamma^{\mu} q_{R \alpha}\right)
\end{aligned}
$$



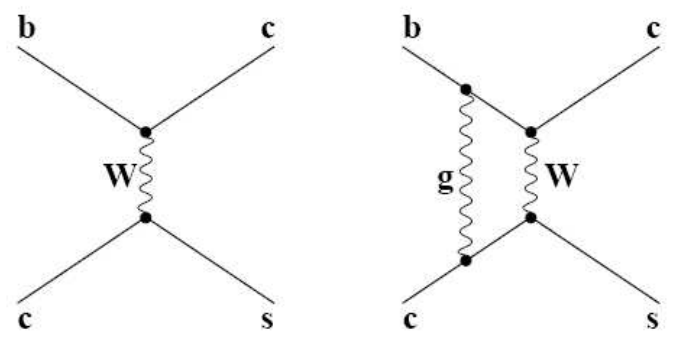

(a)
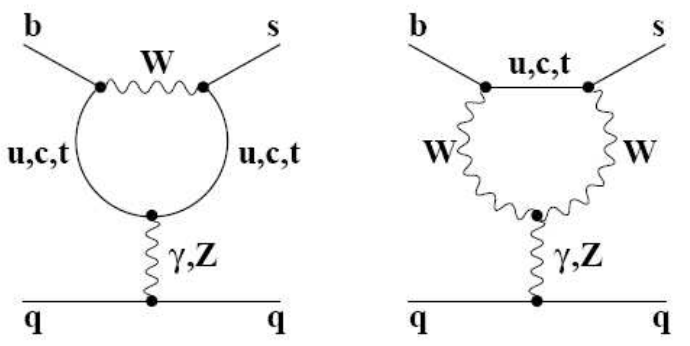

(c)

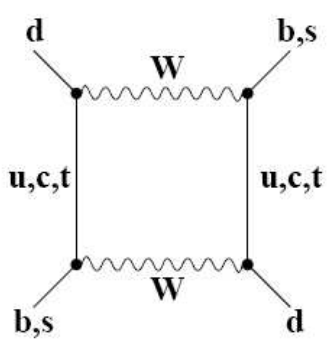

(e)

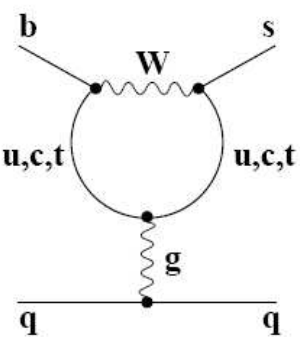

(b)

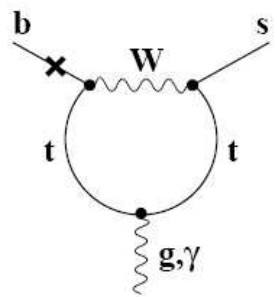

(d)

Figure 2.2: Typical penguin and box diagrams [20].

Electroweak-Penguins (Figure 2.2c):

$$
\begin{aligned}
Q_{3 Q} & =\frac{3}{2}\left(\bar{s}_{L} \gamma_{\mu} b_{L}\right) \sum_{q=u, d, s, c, b} e_{q}\left(\bar{q}_{R} \gamma^{\mu} q_{R}\right) \\
Q_{4 Q} & =\frac{3}{2}\left(\bar{s}_{L \alpha} \gamma_{\mu} b_{L \beta}\right) \sum_{q=u, d, s, c, b} e_{q}\left(\bar{q}_{R \beta} \gamma^{\mu} q_{R \alpha}\right) \\
Q_{5 Q} & =\frac{3}{2}\left(\bar{s}_{L} \gamma_{\mu} b_{L}\right) \sum_{q=u, d, s, c, b} e_{q}\left(\bar{q}_{L} \gamma^{\mu} q_{L}\right) \\
Q_{6 Q} & =\frac{3}{2}\left(\bar{s}_{L \alpha} \gamma_{\mu} b_{L \beta}\right) \sum_{q=u, d, s, c, b} e_{q}\left(\bar{q}_{L \beta} \gamma^{\mu} q_{L \alpha}\right)
\end{aligned}
$$


Magnetic-Penguins (Figure 2.2d):

$$
\begin{aligned}
Q_{7} & =\frac{e}{16 \pi^{2}} m_{b} \bar{s}_{L \alpha} \sigma^{\mu \nu} b_{R \alpha} F_{\mu \nu} \\
Q_{8} & =\frac{g}{16 \pi^{2}} m_{b} \bar{s}_{L \alpha} \sigma^{\mu \nu} T_{\alpha \beta}^{a} b_{R \beta} G_{\mu \nu}^{a} \\
Q_{7^{\prime}} & =\frac{e}{16 \pi^{2}} m_{s} \bar{s}_{R \alpha} \sigma^{\mu \nu} b_{L \alpha} F_{\mu \nu} \\
Q_{8^{\prime}} & =\frac{g}{16 \pi^{2}} m_{s} \bar{s}_{R \alpha} \sigma^{\mu \nu} T_{\alpha \beta}^{a} b_{L \beta} G_{\mu \nu}^{a}
\end{aligned}
$$

$\Delta S=2$ and $\Delta B=2$ Operators (Figure 2.2e):

$$
\begin{aligned}
Q(\Delta S=2) & =\left(\bar{s}_{L} \gamma_{\mu} d_{L}\right)\left(\bar{s}_{L} \gamma^{\mu} d_{L}\right) \\
Q(\Delta B=2) & =\left(\bar{b}_{L} \gamma_{\mu} d_{L}\right)\left(\bar{b}_{L} \gamma^{\mu} d_{L}\right)
\end{aligned}
$$

\section{Semi-Leptonic Operators (Figure 2.2f):}

$$
\begin{aligned}
Q_{9} & =\frac{e^{2}}{16 \pi^{2}}\left(\bar{s}_{L} \gamma_{\mu} b_{L}\right)\left(\bar{l} \gamma^{\mu} l\right) \\
Q_{10} & =\frac{e^{2}}{16 \pi^{2}}\left(\bar{s}_{L} \gamma_{\mu} b_{L}\right)\left(\bar{l} \gamma^{\mu} \gamma_{5} l\right) \\
Q_{9^{\prime}} & =\frac{e^{2}}{16 \pi^{2}}\left(\bar{s}_{R} \gamma_{\mu} b_{R}\right)\left(\bar{l} \gamma^{\mu} l\right) \\
Q_{10^{\prime}} & =\frac{e^{2}}{16 \pi^{2}}\left(\bar{s}_{R} \gamma_{\mu} b_{R}\right)\left(\bar{l} \gamma^{\mu} \gamma_{5} l\right) \\
Q_{\nu \bar{\nu}} & =\frac{\alpha_{e m}}{4 \pi}\left(\bar{s}_{L} \gamma_{\mu} b_{L}\right)\left(\bar{\nu}_{L} \gamma^{\mu} \nu_{L}\right)
\end{aligned}
$$

The quark color indices are represented by $\alpha$ and $\beta$ and are omitted for colour singlet currents and $e_{q}$ is the electric charge of the relevant quark. The subscripts $R$ and $L$ refer to the left- and right-handed components of the fermion field. $F_{\mu \nu}$ and $G_{\mu \nu}^{\alpha}$ are the electromagnetic and strong interaction tensor.

Different decays are sensitive to different Wilson coefficients. For example the transition $b \rightarrow s \ell^{+} \ell^{-}$is sensitive to the values and signs of $C_{7}, C_{9}$ and $C_{10}$ and limits on this coefficients can be obtained by measuring such decays. The calculation of the rare decay rates involves three distinct steps:

- Determination of the initial conditions of the Wilson coefficients at the electroweak scale.

- Evaluation by means of the renormalization group equations of $C_{i}$ down to $\mu=\mathcal{O}\left(m_{b}\right)$

- Evaluation of the hadronic matrix element of the effective operators at $\mu=\mathcal{O}\left(m_{b}\right)$, including both perturbative and non-perturbative QCD corrections. 


\section{$2.4 B \rightarrow X_{s} \gamma$}

It was recognized some time ago that, because of structure of the quark mixing matrix and because of the very heavy top mass [21], rates for processes such as $B \rightarrow X_{s} \gamma$ and $B \rightarrow X_{s} \ell^{+} \ell^{-}$could be sizable. Among rare $B$ decays, the $B \rightarrow X_{s} \gamma$ mode is the most prominent because it was already measured by several independent $e^{+} e^{-}$experiments, mostly at the $\Upsilon(4 S)$ resonance. As this process is dominated by the two-body decay $b \rightarrow s \gamma$, its photon energy spectrum is expected to be a smeared delta function centered at $E_{\gamma} \approx m_{b} / 2$. The effective Hamiltonian is given by

$$
\mathcal{H}_{\mathrm{eff}}=-\frac{4 G_{F}}{\sqrt{2}} V_{t s}^{*} V_{t b} \sum_{i=1}^{8} C_{i}(\mu) Q_{i}(\mu)
$$

The operators contributing to the effective Hamiltonian are the current-current operators $\left(Q_{1}, Q_{2}\right)$, the QCD-penguin operators $\left(Q_{3}, \ldots, Q_{6}\right)$ and the magnetic penguin operators $\left(Q_{7 \gamma}, Q_{8 G}\right)$. It is the magnetic $\gamma$-penguin that plays the crucial role in this decay. However, the role of the dominant current-current operator $Q_{2}$ should not be underestimated. Indeed the short distance QCD effects involving in particular the mixing between $Q_{2}$ and $Q_{7 \gamma}$ are very important in this decay. They are known to enhance $C_{7 \gamma}(\mu)$ substantially, so that the resulting branching fraction $\operatorname{Br}\left(B \rightarrow X_{s} \gamma\right)$ turns out to be a factor 2-3 higher than it would be without QCD effects [22, 23]. At next-to-next-to-leading order QCD the theoretical SM branching fraction is predicted to be [24]

$$
\mathcal{B}\left(B \rightarrow X_{s} \gamma\right)_{S M}=(3.15 \pm 0.23) \times 10^{-4}
$$

for $E_{\gamma}>1.6 \mathrm{GeV}$ in the B-meson rest frame. The latest measurements were performed by Belle [25] and BABAR [26] and the world average performed by the Heavy Flavour Averaging Group [27] for $E_{\gamma}>1.6 \mathrm{GeV}$ is

$$
\mathcal{B}\left(B \rightarrow X_{s} \gamma\right)_{\text {exp }}=(3.52 \pm 0.23 \pm 0.09) \times 10^{-4}
$$

which is in good agreement with theory.

\section{$2.5 \quad B \rightarrow X_{s} \ell^{+} \ell^{-}$}

The inclusive $B \rightarrow X_{s} \ell^{+} \ell^{-}$decay presents a complementary and a more complex test of the SM, since different contributions add to the decay rate. It is particularly attractive because of kinematic observables such as the invariant dilepton mass spectrum and the forward-backward (FB) asymmetry. The effective Hamiltonian of this decay is given by

$$
\mathcal{H}_{\mathrm{eff}}\left(b \rightarrow s \mu^{+} \mu^{-}\right)=\mathcal{H}_{\mathrm{eff}}(b \rightarrow s \gamma)-\frac{4 G_{F}}{\sqrt{2}} V_{t s}^{*} V_{t b}\left[C_{9 V}(\mu) Q_{9 V}+C_{10 A}(\mu) Q_{10 A}\right]
$$


The semileptonic $Z^{0}$ and the $\gamma$ penguin operators $\left(Q_{9 V}, Q_{10 A}\right)$ are additional contributions compared to $\mathcal{H}_{\text {eff }}(b \rightarrow s \gamma)$. The SM values of the Wilson coefficients can be found in Table 2.1 [28].

Table 2.1: Values of SM Wilson coefficients. Here, $C_{7}^{\text {eff }} \equiv C_{7}-C_{5} / 3-C_{6}$ ) and $C^{(0)} \equiv$ $3 C_{1}+C_{2}+3 C_{3}+C_{4}+3 C_{5}+C_{6}[28]$.

\begin{tabular}{|c|c|c|c|c|c|c|c|c|}
\hline$C_{1}$ & $C_{2}$ & $C_{3}$ & $C_{4}$ & $C_{5}$ & $C_{6}$ & $C_{7}^{\text {eff }}$ & $C_{9}$ & $C_{10}$ \\
\hline-0.248 & +1.107 & +0.011 & -0.026 & +0.007 & -0.031 & -0.313 & +4.344 & -4.669 \\
\hline
\end{tabular}

By precise measurements of the forward-backward asymmetry in $b \rightarrow s \ell^{+} \ell^{-}$the Wilson coefficients $C_{7}, C_{9}$, and $C_{10}$ and their signs can be determined. This helps to consolidate the SM or indicate New Physics (see Fig. 2.3). The forward-backward asymmetry

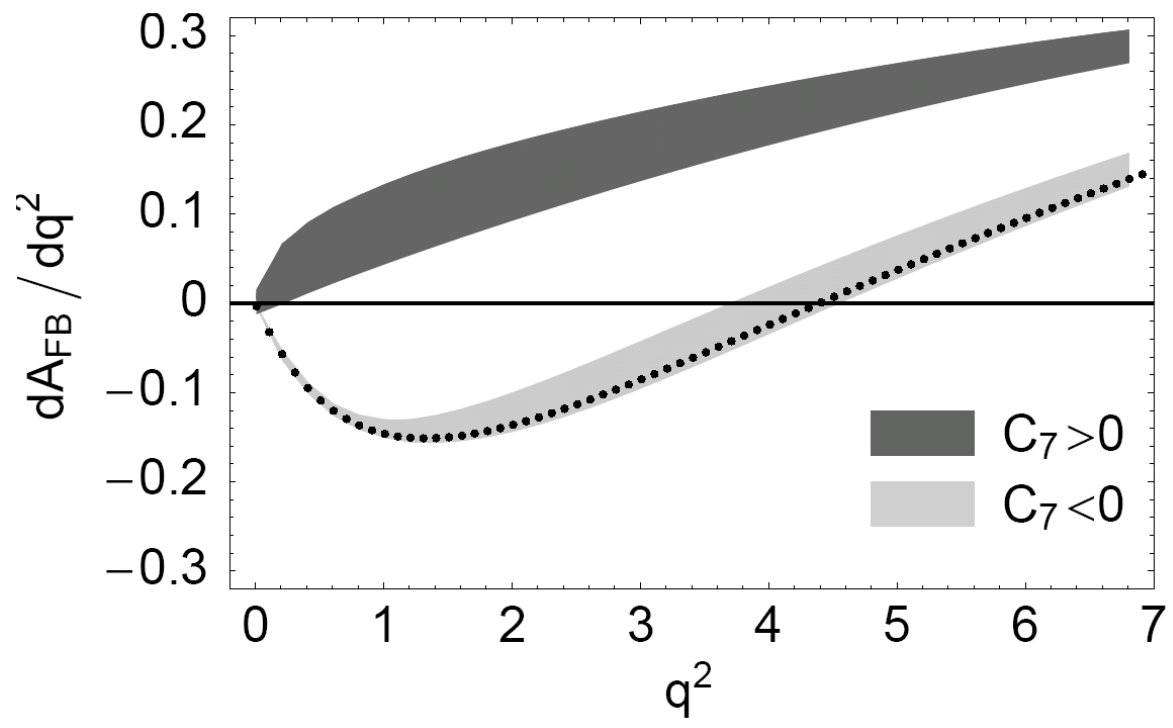

Figure 2.3: FB asymmetry for the decay $B \rightarrow K^{*} \ell^{+} \ell^{-}$as a function of $q^{2}$. The light band corresponds to the region of parameter space with same (negative) sign for $C_{7}$ as in the SM; the dark band refers to solutions with flipped sign for $C_{7}$; the dotted line is the central value of the SM prediction [29]. 
for leptons as a function of the squared di-lepton mass $m_{\ell \ell}^{2}=q^{2}$ is defined as

$$
A_{F B}\left(q^{2}\right)=\frac{\int_{0}^{1} \frac{d^{2} \Gamma}{d x d q^{2}} d x-\int_{-1}^{0} \frac{d^{2} \Gamma}{d x d q^{2}} d x}{\frac{d \Gamma}{d q^{2}}}
$$

where $x \equiv \cos \theta$ parameterises the angle between the $\ell^{+}$and the $B$ meson in the di-lepton centre-of-mass frame.

The long-distance contributions are generated by the current-current operators $Q_{1}$ and $Q_{2}$. In the dimuon invariant mass regions around the $J / \psi$ and $\psi(2 S)$ resonances, the decay is largely dominated by the long-distance contributions, while the short-distance contributions dominate away from these resonances. The branching fraction of the resonant long-distance decay is about two to three orders of magnitude larger than the shortdistance decay (see Fig. 2.4). For the calculation of the non-resonant branching fraction the large distance processes have to be excluded. For most final states, the long-distance decay $B \rightarrow X_{s} J / \psi$ is well established (with the $J / \psi$ usually seen via its decay to two muons), and the main interest lies in the observation of the short distance processes. To

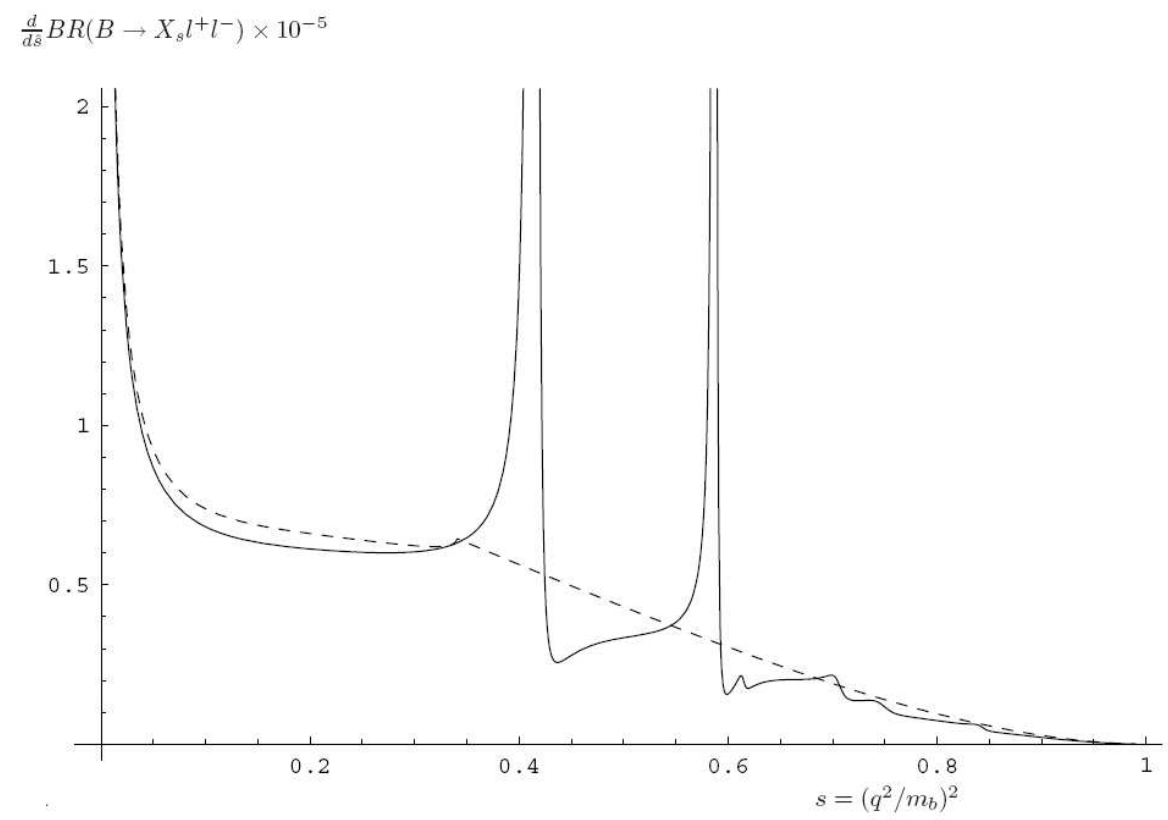

Figure 2.4: Schematic dilepton mass spectrum of $B \rightarrow X_{s} \ell^{+} \ell^{-}$, the dashed line corresponds to the perturbative contribution [30]. The solid line shows the two resonances of $J / \psi$ and $\psi^{\prime}$ 
evaluate the short-distance physics in the di-lepton mass spectrum two regions are interesting:

- low $q^{2}$ region between 1 and $6 \mathrm{GeV} / c^{2}$ with a reliable $q^{2}$ spectra and small $1 / m_{b}$ corrections. Here, the decay rate is sensitive to the interference of $C_{7}$ and $C_{9}$.

- high $q^{2}$ region where the $1 / m_{b}$ corrections are more important and with a less reliable $q^{2}$ spectra. Here, it is easier to perform a inclusive measurement although the rate is lower. The branching fraction in this region is mainly sensitive to $\left|C_{10}\right|^{2}$

\subsubsection{Exclusive decays}

It is experimentally easier to measure the exclusive decays $B \rightarrow K \ell^{+} \ell^{-}$or $B \rightarrow$ $K^{*} \ell^{+} \ell^{-}$than to perform an inclusive measurement. The uncertainties on the SM predictions are dominated by the errors on the hadronic form factors and are much larger than in the corresponding inclusive decays. Different methods have been used to calculate the form factors. Usually they are calculated in a small $q^{2}$ region and then extrapolated to the entire region. Ali et al. used in their calculations the Light Cone-QCD sum rule approach while Melikhov et al. used the quark model. The results of their calculations for the branching fractions can be seen in Table 2.2. Due to the theoretical uncertainties in the determination of the form factor, it is difficult to obtain fundamental parameters of the SM, such as CKM elements, from the exclusive decays.

Table 2.2: Branching fractions for $B \rightarrow K^{(*)} \ell^{+} \ell^{-}$predicted in the framework of the Standard Model.

\begin{tabular}{lcc}
\hline \hline \multirow{2}{*}{ Mode } & \multicolumn{2}{c}{ Predicted branching fraction $\mathcal{B}\left[\times 10^{-6}\right]$} \\
& Ali et al. [31] & Melikhov et al. [32] \\
\hline \hline$B^{ \pm} \rightarrow K \ell^{+} \ell^{-}$ & $0.35 \pm 0.12$ & 0.44 \\
$B \rightarrow K^{*} e^{+} e^{-}$ & $1.58 \pm 0.49$ & 1.5 \\
$B_{d}^{0} \rightarrow K^{*} \mu^{+} \mu^{-}$ & $1.19 \pm 0.39$ & 1.15 \\
\hline \hline
\end{tabular}

The exclusive decays have been measured at hadron colliders and at B-factories. The first observation of $B \rightarrow K \ell^{+} \ell^{-}$was reported in 2002 by the Belle collaboration [33]. Also the decay $B \rightarrow K^{*} \ell^{+} \ell^{-}$was first observed by the Belle experiment, in 2003 [34]. Shortly after, also the BaBar collaboration reported measurements of these exclusive decays [35]. The most recent measurements are summarized in Table 2.3 [27]. 
Table 2.3: Measurements of the branching fractions for $B \rightarrow K^{(*)} \ell^{+} \ell^{-}$.

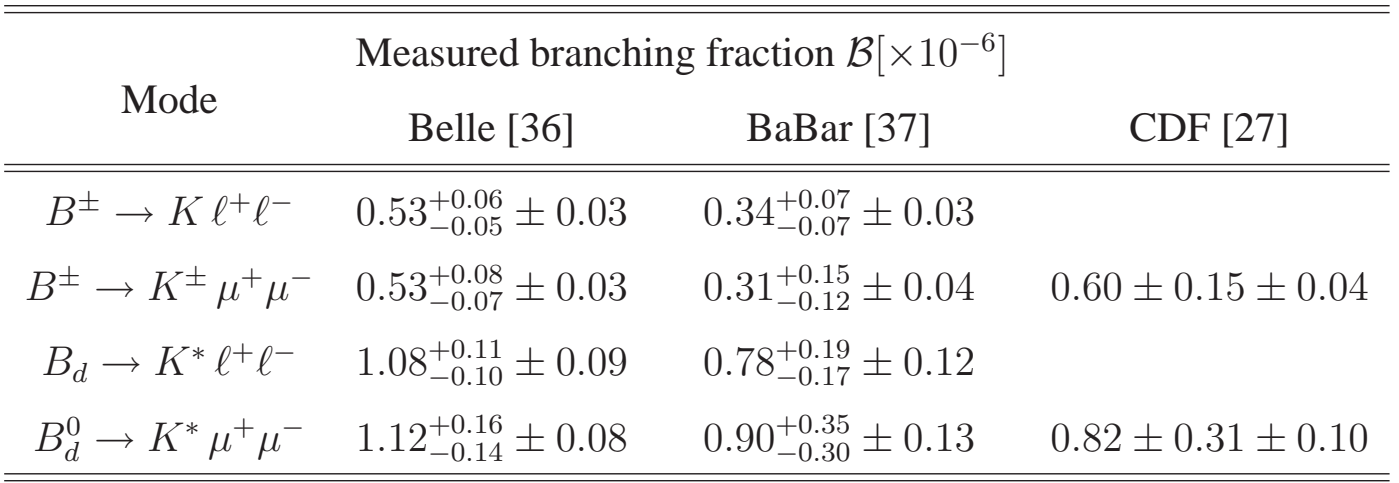

As discussed in the previous chapter, the forward-backward asymmetry $A_{F B}$ is an interesting kinematic property. The Belle collaboration reported in [38] the first measurement of the forward-backward asymmetry as a function of $q^{2}$ for $B \rightarrow K^{*} \ell^{+} \ell^{-}$. Within their limited statistical precision, the measured asymmetry is consistent with the SM. In a more recent Belle publication [36] the forward-backward asymmetry tends to be shifted toward the positive side from the SM expectation (see Fig. 2.3).

\subsection{Normalisation processes}

When observing a decay of a $B$ meson to a specific final state $f$ the expected number of events is given by

$$
N_{o b s}(f)=\int \mathcal{L} d t \cdot \sigma_{B} \cdot \mathcal{B}(B \rightarrow f) \cdot \epsilon
$$

where $\int \mathcal{L} d t$ is the total integrated luminosity of the data sample used in the analysis, $\sigma_{B}$ is the $B$ meson production cross section, $\mathcal{B}(B \rightarrow f)$ is the branching fraction into the specific final state and $\epsilon$ is the corresponding detection and reconstruction efficiency. The fragmentation of the $b \bar{b}$ to the different $B$ mesons has to be taken into account and is given by

$$
\begin{aligned}
\sigma_{B^{ \pm}} & =\sigma_{b \bar{b}} \cdot f\left(\bar{b} \rightarrow B^{ \pm}\right) \\
\sigma_{B_{d}} & =\sigma_{b \bar{b}} \cdot f\left(\bar{b} \rightarrow B_{d}\right)
\end{aligned}
$$

where $\sigma_{b \bar{b}}$ is the total $b \bar{b}$ production cross section which has to be multiplied by the fragmentation fraction $f$ of producing a $B^{ \pm}$or a $B_{d}$. The expected number of events for the 
decays $B^{ \pm} \rightarrow K^{ \pm} \mu^{+} \mu^{-}$and $B^{ \pm} \rightarrow J / \psi K^{ \pm}$is then given by:

$$
\begin{aligned}
N_{B^{ \pm} \rightarrow J / \psi K^{ \pm}} & =\int \mathcal{L} d t \cdot \sigma_{B^{ \pm}} \cdot \mathcal{B}\left(B^{ \pm} \rightarrow J / \psi K^{ \pm}\right) \cdot \mathcal{B}\left(J / \psi \rightarrow \mu^{+} \mu^{-}\right) \cdot \epsilon_{J / \psi K}^{B^{ \pm}} \\
N_{B^{ \pm} \rightarrow K^{ \pm} \mu^{+} \mu^{-}} & =\int \mathcal{L} d t \cdot \sigma_{B^{ \pm}} \cdot \mathcal{B}\left(B^{ \pm} \rightarrow K^{ \pm} \mu^{+} \mu^{-}\right) \cdot \epsilon_{\mu^{+} \mu^{-} K}^{B^{ \pm}}
\end{aligned}
$$

By the normalisation of the measurement to a decay with a similar final state the total luminosity an the B production cross section cancel out. The ratio of the two Equations 2.14 is:

$$
\frac{N_{B^{ \pm} \rightarrow K^{ \pm} \mu^{+} \mu^{-}}}{N_{B^{ \pm} \rightarrow J / \psi K^{ \pm}}}=\frac{\mathcal{B}\left(B^{ \pm} \rightarrow K^{ \pm} \mu^{+} \mu^{-}\right) \cdot \epsilon_{\mu^{+} \mu^{-} K}^{B^{ \pm}}}{\mathcal{B}\left(B^{ \pm} \rightarrow J / \psi K^{ \pm}\right) \cdot \mathcal{B}\left(J / \psi \rightarrow \mu^{+} \mu^{-}\right) \cdot \epsilon_{J / \psi K^{ \pm}}^{B^{ \pm}}}
$$

From Equation 2.15, one can derive a formula to calculate the branching fraction or a limit for the decay $B^{ \pm} \rightarrow K^{ \pm} \mu^{+} \mu^{-}$

$\mathcal{B}\left(B^{ \pm} \rightarrow K^{ \pm} \mu^{+} \mu^{-}\right)=\frac{N_{B^{ \pm} \rightarrow K^{ \pm}} \mu^{+} \mu^{-}}{N_{B^{ \pm} \rightarrow J / \psi K^{ \pm}}} \cdot \frac{\epsilon_{J / \psi K^{ \pm}}^{B^{ \pm}}}{\epsilon_{\mu^{+} \mu^{-} K}^{B^{ \pm}}} \cdot \mathcal{B}\left(B^{ \pm} \rightarrow J / \psi K^{ \pm}\right) \cdot \mathcal{B}\left(J / \psi \rightarrow \mu^{+} \mu^{-}\right)$

The same holds for the decay $B_{d}^{0} \rightarrow K^{*} \mu^{+} \mu^{-}$using $B_{d}^{0} \rightarrow J / \psi K^{*}(892)$ as normalisation channel. The decays $B^{ \pm} \rightarrow J / \psi K^{ \pm}$and $B_{d}^{0} \rightarrow J / \psi K^{*}(892)$ occur at tree level (see Fig. 2.5) and therefore the branching fractions are about $10^{3}$ times larger than for the rare decays $B^{ \pm} \rightarrow K^{ \pm} \mu^{+} \mu^{-}$and $B_{d}^{0} \rightarrow K^{*} \mu^{+} \mu^{-}$(see Table 2.4). The normalisation channel is also used for comparisons between data and simulation as it should have similar kinematic properties as the signal channel and a larger branching fraction. .

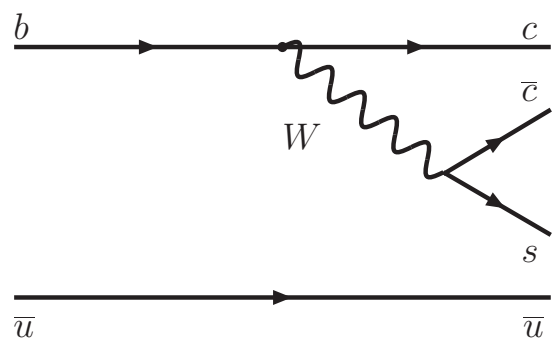

Figure 2.5: SM diagrams for the decay $B^{ \pm} \rightarrow J / \psi K^{ \pm}$. 
Table 2.4: The branching fraction for the signal and normalisation channel. The values are taken from the PDG [14].

\begin{tabular}{cc}
\hline \hline Decay Channel & Branching fraction \\
\hline$B^{ \pm} \rightarrow K^{ \pm} \mu^{+} \mu^{-}$ & $\left(4.5_{-1.7}^{+1.4}\right) \cdot 10^{-7}$ \\
$B^{ \pm} \rightarrow J / \psi K^{ \pm}$ & $(1.00 \pm 0.04) \cdot 10^{-3}$ \\
\hline$B_{d}^{0} \rightarrow K^{*} \mu^{+} \mu^{-}$ & $\left(1.10_{-0.26}^{+0.29}\right) \cdot 10^{-6}$ \\
$B_{d}^{0} \rightarrow J / \psi K^{*}(892)$ & $(1.33 \pm 0.06) \cdot 10^{-3}$ \\
\hline \hline
\end{tabular}




\section{Chapter 3}

\section{The DØ experiment at the Tevatron}

The Tevatron proton-antiproton $(p \bar{p})$ collider at the Fermi National Accelerator Laboratory (FERMILAB) [39] in Batavia, Illinois, USA, is the highest-energy particle collider currently operational (see Fig. 3.1). The two experiments DØ [40] and CDF [41] collected about $125 \mathrm{pb}^{-1}$ of $p \bar{p}$ collision data at a center-of-mass energy of $1.8 \mathrm{TeV}$ during the years 1992 to 1996 (Run I), leading to the discovery of the top quark [42, 43] and the measurement of its mass. Further a precision measurement of the mass of the $W$ boson, detailed analyses of gauge boson couplings, studies of jet production and vastly improved limits on new phenomena, such as leptoquarks and supersymmetric particles, among many other measurements have been accomplished.

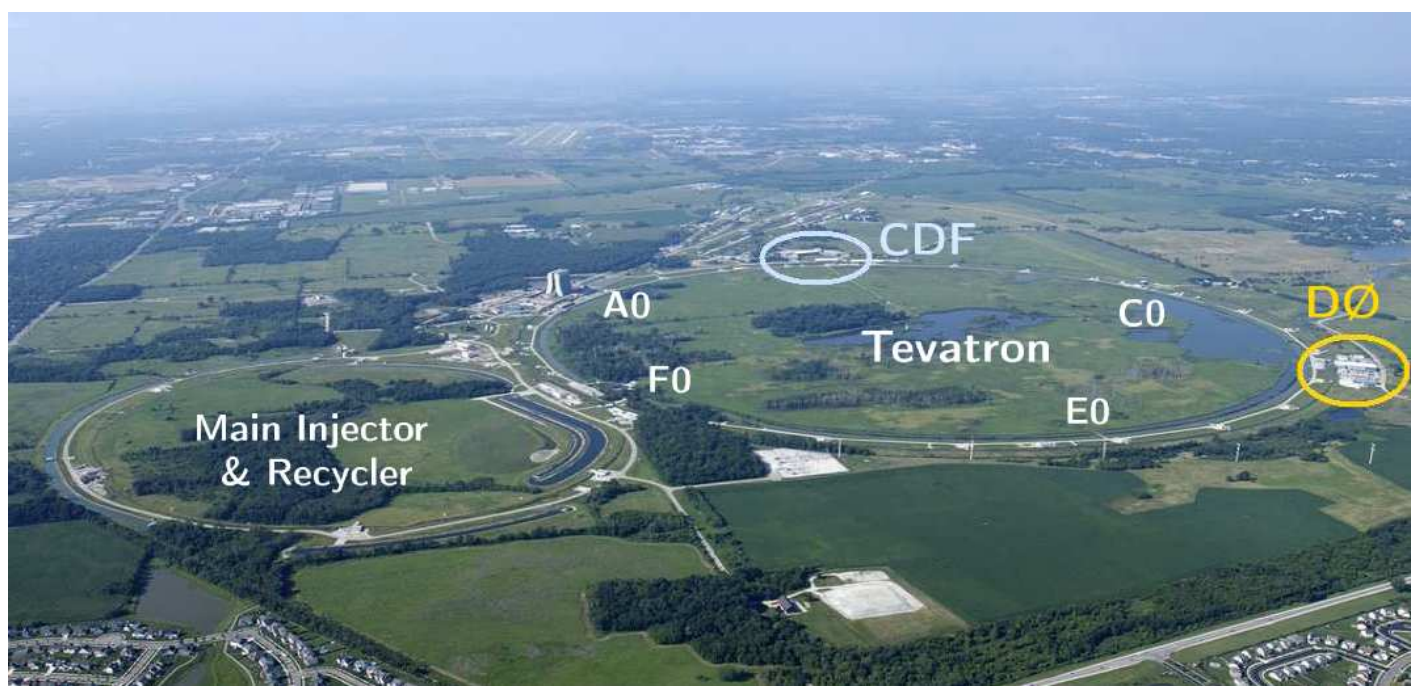

Figure 3.1: The accelerator facilities at FERMILAB with the two multi purpose detectors $\mathrm{CDF}$ and $\mathrm{D} \emptyset$. 
During a shutdown of five years the accelerator and the experiments were significantly upgraded [44, 45]. In 2001 the second data-taking period started (Run II) and it is expected to collect around $8 \mathrm{fb}^{-1}-9 \mathrm{fb}^{-1}$ by the year 2009 .

\subsection{Tevatron}

After the upgrade the Tevatron accelerator operates at a center-of-mass energy of $1.96 \mathrm{TeV}$. The main ring has a circumference of about $6 \mathrm{~km}$ in which the protons circulate clockwise and the antiprotons counter clockwise. In two interaction regions, where the detectors of $\mathrm{CDF}$ and $\mathrm{D} \varnothing$ are built, the particles collide.

There are several stages to complete this process, starting from producing the protons and antiprotons, successive acceleration and finally colliding them at the two detector points. The Fermilab accelerator facilities are shown in Fig 3.2

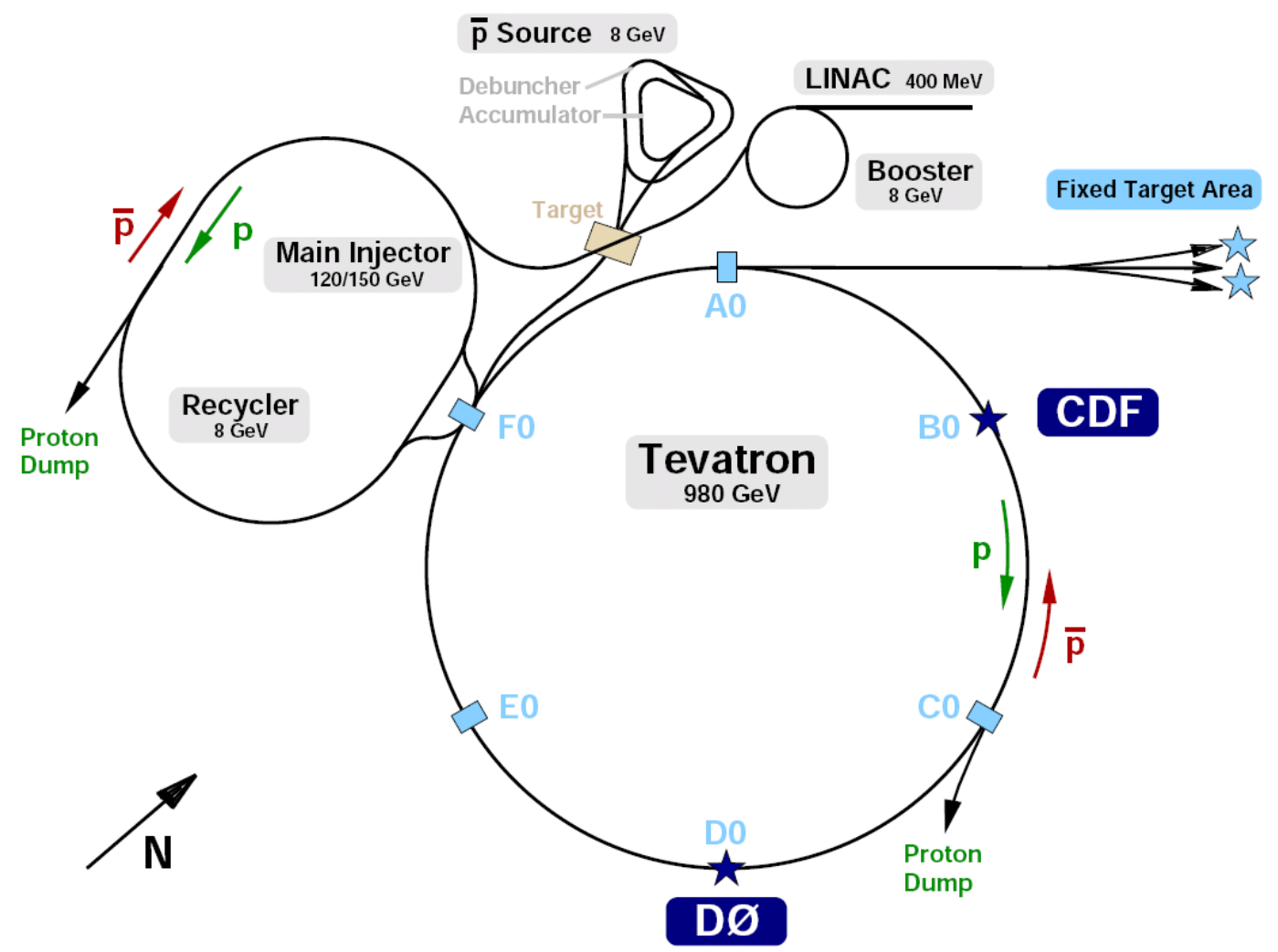

Figure 3.2: The accelerator facilities at FERMILAB with the two multi purpose detectors $\mathrm{CDF}$ and $\mathrm{D} \varnothing$. 


\subsubsection{Production of protons and pre-acceleration}

Negatively charged hydrogen ions are accelerated to $750 \mathrm{keV}$ by a Cockcroft-Walton accelerator and then injected into a $165 \mathrm{~m}$ long linear accelerator which boosts their energy to $400 \mathrm{MeV}$. The $\mathrm{H}^{-}$ions pass through a carbon foil to strip off their two electrons and leave $\mathrm{H}^{+}$ions (protons) which are injected into the Booster, a synchrotron which brings their energy to $8 \mathrm{GeV}$. From the Booster the protons are sent to the Main Injector for the next stage of acceleration.

\subsubsection{Main Injector (MI)}

The Main Injector performs several functions besides accelerating protons and antiprotons to an energy of $150 \mathrm{GeV}$ and injecting them into the Tevatron [46]. The $8 \mathrm{GeV}$ protons from the Booster are first accelerated to $120 \mathrm{GeV}$ and then delivered to the antiproton production target (a Nickel-Copper target). The collision energy is chosen such that the energy spectrum of the emerging antiprotons $(\bar{p})$ has its maximum at about $8 \mathrm{GeV}$. On average, around 50000 protons are necessary to produce one antiproton in the required energy range. The Tevatron complex is designed in such a way that new antiprotons can be produced in parallel to collisions taking place in the main Tevatron ring. The produced antiprotons are cooled and debunched in the Debuncher and Accumulator, and once the number of antiprotons is sufficiently large they are passed back to the MI where they are accelerated to $150 \mathrm{GeV}$ before being transfered to the Tevatron.

\subsubsection{The Tevatron Ring Synchrotron}

In the last stage of the acceleration process the $150 \mathrm{GeV}$ protons and antiprotons from the MI are passed in bunches into the Tevatron ring. 36 bunches of protons and 36 bunches of antiprotons are loaded in opposite directions which results in a time interval of $396 \mathrm{~ns}$ between two consecutive collisions. After the injection the particles are accelerated to their final energy of $980 \mathrm{GeV}$, squeezed in the transverse plane through the quadrupole magnets and collided at the two intersection points.

The number of collisions per second depends on the instantaneous luminosity. Figure 3.3 shows the integrated luminosity per week and the total integrated luminosity accumulated in Run II from May 2001 until August 2008 as of today an integrated luminosity of $5 \mathrm{fb}^{-1}$. 


\section{Collider Run II Integrated Luminosity}

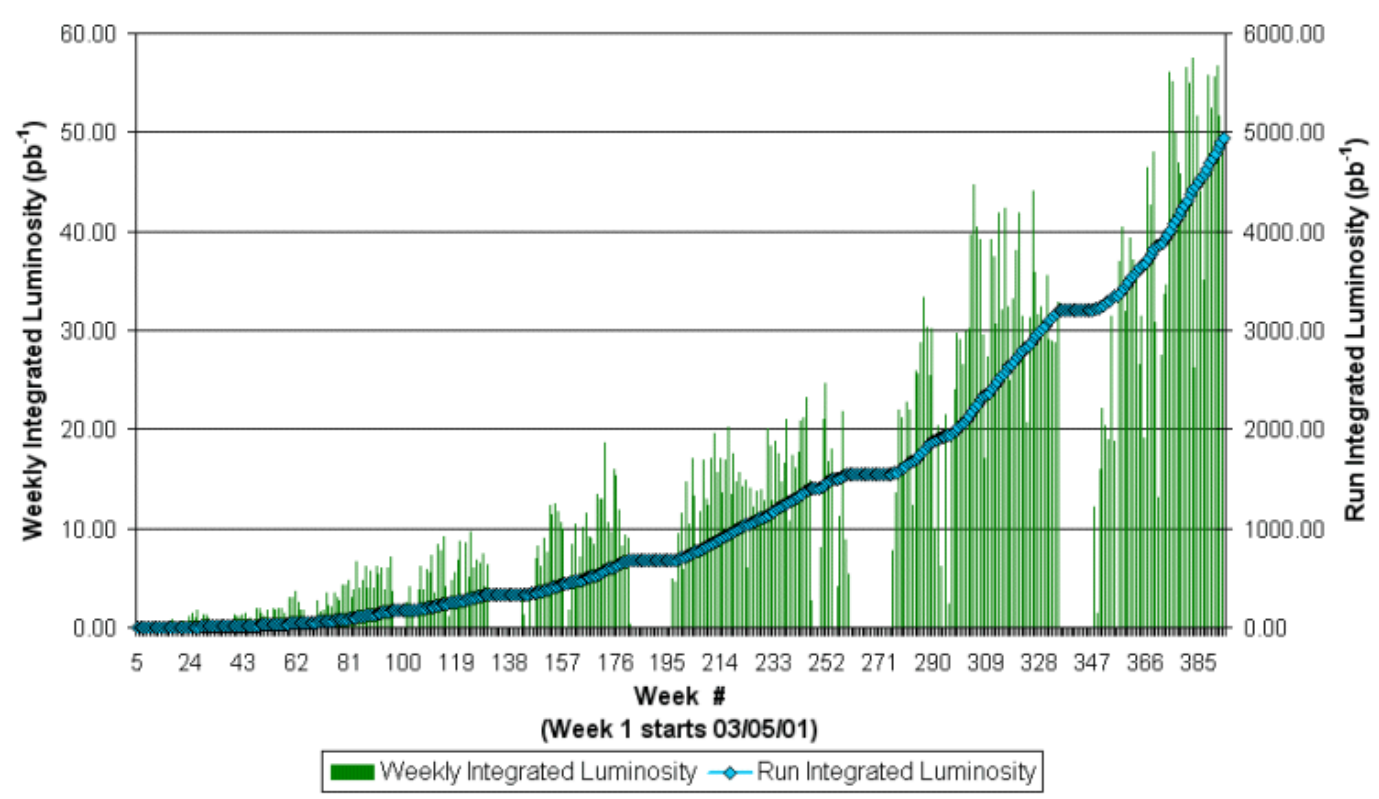

Figure 3.3: The integrated luminosity per week and total integrated luminosity for Run II

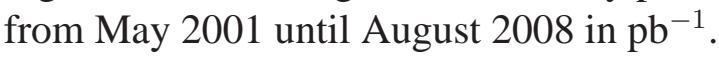

\subsection{The DØ Detector}

The $\mathrm{D} \varnothing$ detector is a multi-purpose detector designed to measure the remnants of $p \bar{p}$ collisions, especially muons, electrons, jets and missing transverse energy. It was constructed in the late 1980's and early 1990's. After the period from 1992 to 1996 (Run I) the DØ detector was upgraded to handle the increased luminosity of the Tevatron $[47,48]$ in Run II. The DØ detector, like other high energy particle physics detectors, is composed of different sub-systems that surrounds each other like the layers of an onion. There are three major sub-systems. In the core of the detector, there is the tracking system surrounded by a 2 Tesla solenoid magnetic field. This combination allows precise measurements of the positions and transverse momenta of the charged particles traveling outwards from the interaction point. Outside of the tracking volume and the solenoid, there are the D $\varnothing$ calorimeters, a system of finely grained Uranium and Liquid Argon calorimeters which record the energies and positions of hadronic and electromagnetic showers. The last subsystem is a three layer muon spectrometer which detects the presence and measures the momenta of the muons escaping the detector. Figure 3.4 shows a schematic overview of the detector. 


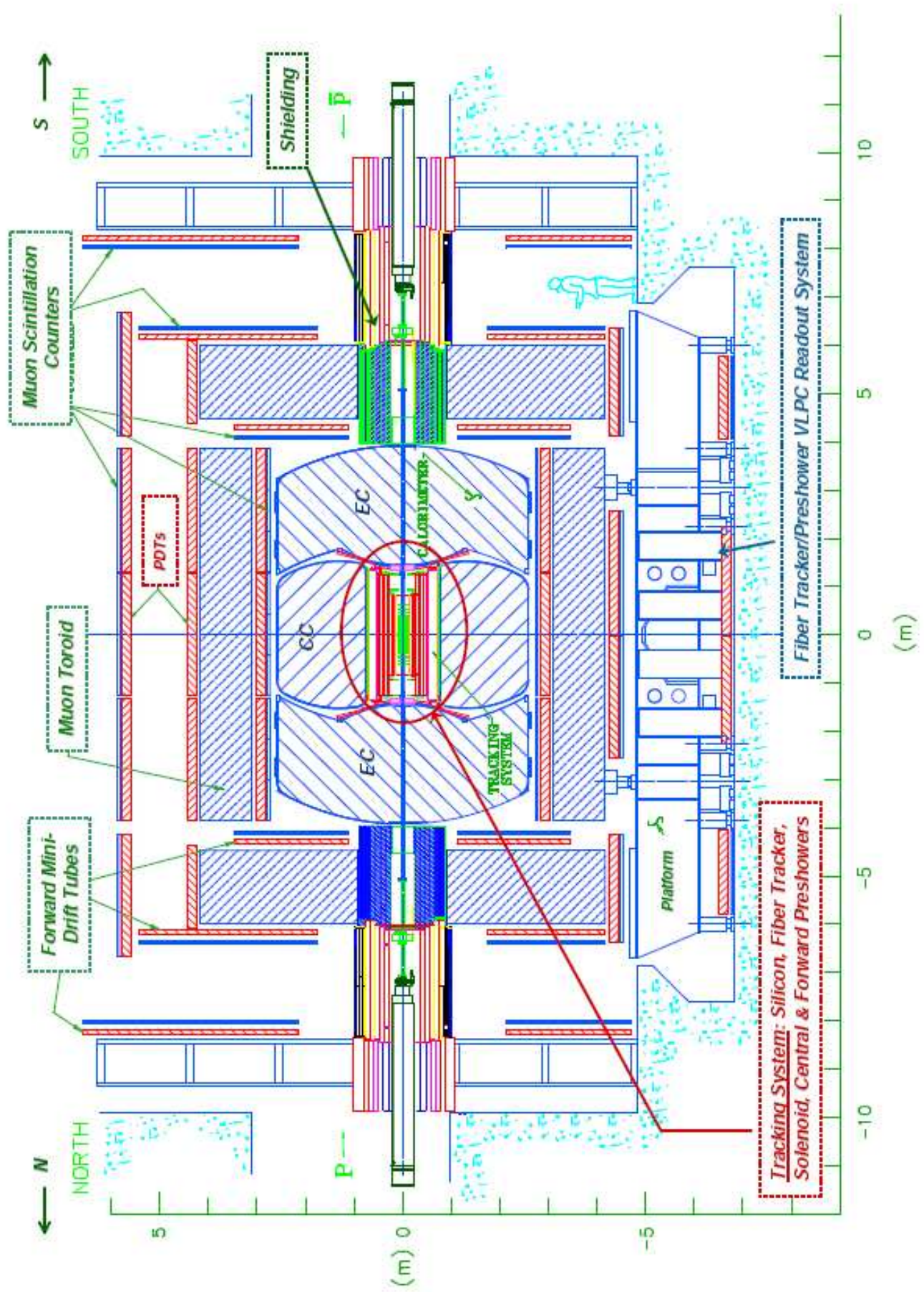

Figure 3.4: Cross-sectional view of the DØ Run II detector. 


\subsubsection{The coordinate System}

$\mathrm{D} \varnothing$ uses a right-handed coordinate system where the proton beam defines the positive $z$ axis. The $y$-axis points upwards and the $x$-axis points towards the center of the Tevatron ring. There are two common choices for the origin of the coordinate system: The physics coordinates, where the origin is located at the reconstructed vertex of the interaction, and the detector coordinates, where the origin is chosen to be at the center of the $\mathrm{D} \varnothing$ detector. Because the D $\varnothing$ detector has cylindrical symmetry around the $z$-axis it is often useful to use cylindrical coordinates $(r, \phi)$ with the transformation:

$$
\begin{aligned}
r & =\sqrt{x^{2}+y^{2}} \\
\phi & =\arctan \frac{y}{x}
\end{aligned}
$$

Additionally, it is sometimes convenient to introduce the pseudorapidity $\eta$, which can be expressed with the polar angle $\theta$ :

$$
\eta=-\ln \tan \frac{\theta}{2}
$$

\subsubsection{Central Tracking System}

The central tracking system (see Fig. 3.5) consists of an inner high resolution silicon tracker (Layer 0 and SMT) surrounded by a scintillating central fiber tracker (CFT). These detectors are immersed in a 2 Tesla magnetic solenoid field. The tracking system was designed to provide momentum measurements using the magnetic field good electron identification, tracking over a large pseudorapidity range, secondary vertex detection, triggering, and a fast response for a bunch crossing time of $396 \mathrm{~ns}$.

\section{Silicon Microstrip Tracker - SMT}

The SMT uses the silicon $p n$-junction technology with readout strips to perform precise position measurements with a resolution of about $20 \mu \mathrm{m}$. In order to allow good vertexing and three-dimensional track reconstruction for higher momentum tracks over a large acceptance up to an $|\eta \approx| 3$, a layout was chosen with six barrel modules with rectangular silicon sensors parallel to the beam axis, interspersed with twelve F-disks with wedgeshaped sensors transverse to the beam axis (see Fig. 3.6). In the forward and backward region are two more disks, the so-called H-disks. While the barrel detector measures primarily the $r-\phi$ coordinates, the disks measure both $r-\phi$ and $r-z$.

Each barrel is built of silicon 4 detector layers that can be seen in Fig. 3.7. The two layers 1 and 3 of the four inner barrels are double-sided silicon ladders with axial strips 


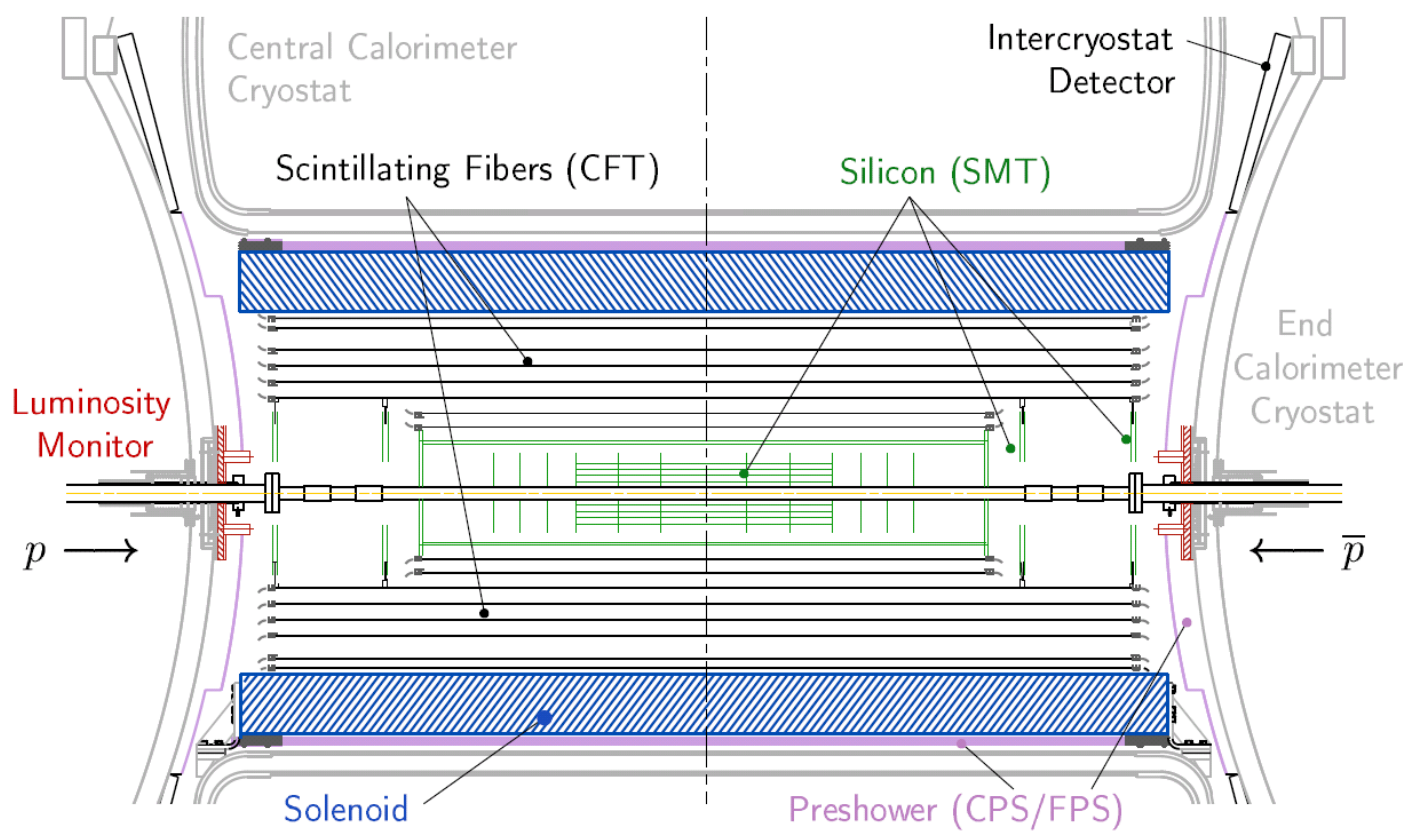

Figure 3.5: Schematic view of the inner tracking system in the $(y, z)$-plane. Depicted are the tracking detectors SMT and CFT, located inside the 2 Tesla solenoid field.

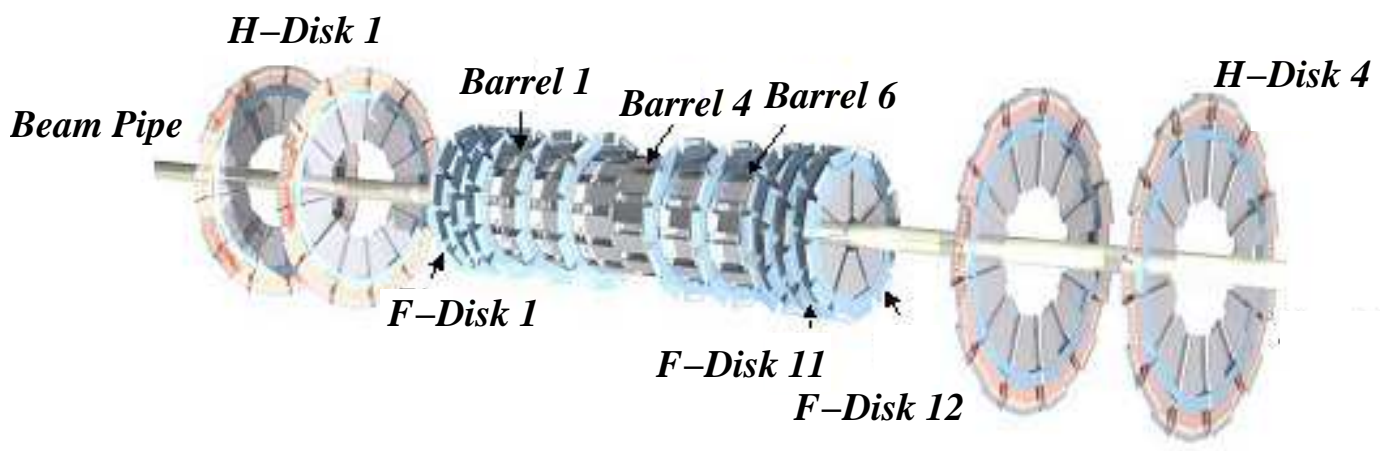

Figure 3.6: Isometric view of the DØ Silicon Microstrip Tracker.

(parallel to the beam line) on one side and $90^{\circ}$ stereo angle strips on the other side, while the outer barrels have only single-sided ladders with axial strips and hence provide no stereo information. Layers 2 and 4 of all barrels are equipped with double-sided silicon sensors with axial strips on one side and $2^{\circ}$ stereo angle on the reverse side.

The F-disks are made of double-sided wedges with $-15^{\circ}$ stereo angle on one side and $+15^{\circ}$ on the other. Four F-disks are sandwiched between the barrels while the remaining 


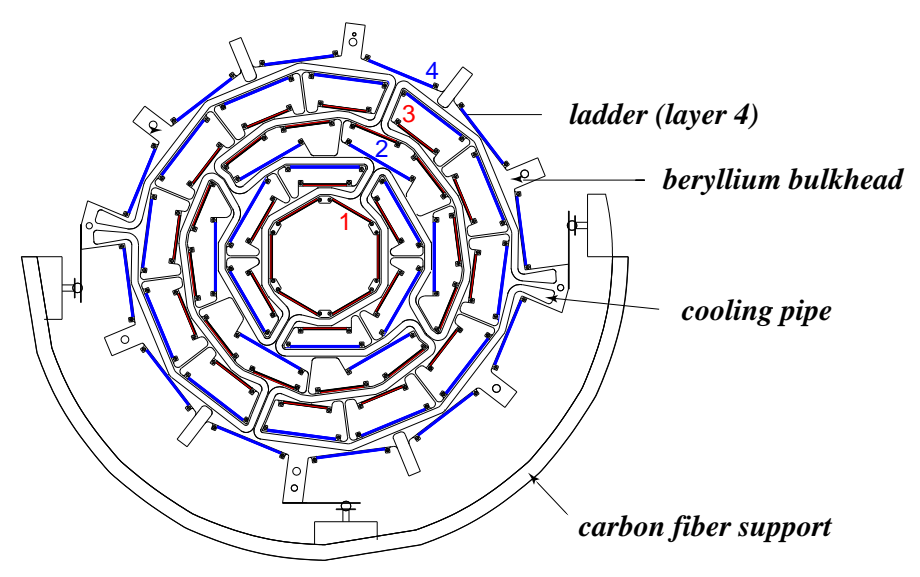

Figure 3.7: Cross section of an an DØ SMT barrel with the 4 detector layers build of single or double-sided ladders (ladders) .

eight are located at both ends of the SMT barrel. Towards the end of the interaction region, there are the H-disks, two on each side. These disks are made of single-sided wedges and help to extend the SMT coverage to $\left|\eta_{\text {det }}\right|<3.0$. A summary of the SMT layout is given in Table 3.1 [49].

\begin{tabular}{llll}
\hline \hline & Barrels & F-Disks & H-Disks \\
\hline \hline \#Channels & 387072 & 258048 & 147456 \\
Sensors & s/d sided & double sided & single sided \\
Stereo & $0^{\circ}, 2^{\circ}, 90^{\circ}$ & $\pm 15^{\circ}$ & $\pm 7.5^{\circ}$ \\
\#Modules & 432 & 144 & 96 pairs \\
Si area & $1.3 \mathrm{~m}^{2}$ & $0.4 \mathrm{~m}^{2}$ & $1.3 \mathrm{~m}^{2}$ \\
Inner radius & $2.7 \mathrm{~cm}$ & $2.6 \mathrm{~cm}$ & $9.5 \mathrm{~cm}$ \\
Outer radius & $9.4 \mathrm{~cm}$ & $10.5 \mathrm{~cm}$ & $26 \mathrm{~cm}$ \\
Maximal $|z|$ & $38.4 \mathrm{~cm}$ & $54.8 \mathrm{~cm}$ & $120 \mathrm{~cm}$ \\
\hline \hline
\end{tabular}

Table 3.1: Specifications of the silicon vertex detector, where "s, d" means single, double sided. 


\section{Central Fiber Tracker - CFT}

The Central Fiber Tracker [47, 48] surrounds the SMT what can be seen in Fig. 3.5 and 3.8 and covers the central region to $\left|\eta_{\text {det }}\right|<2.0$. The CFT is composed of approximately $200 \mathrm{~km}$ of scintillating fiber [50] with a diameter of $830 \mu \mathrm{m}$. The scintillating fibers are arranged in 8 barrels between radii of $20 \mathrm{~cm}$ and $60 \mathrm{~cm}$. Each barrel consists of two double layers of fibers, one axial double layer and one stereo double layer of either $+3^{\circ}$ or $-3^{\circ}$ stereo fibers. The fibers in each layer are grouped into "ribbons". The number of ribbons varies from 10 in the first CFT layer to 28 in the last layer. To provide maximal coverage, the fibers of a double layer are staggered with an offset of half a fiber width as shown in Fig. 3.8. The resolution of the CFT is about $100 \mu \mathrm{m}$.

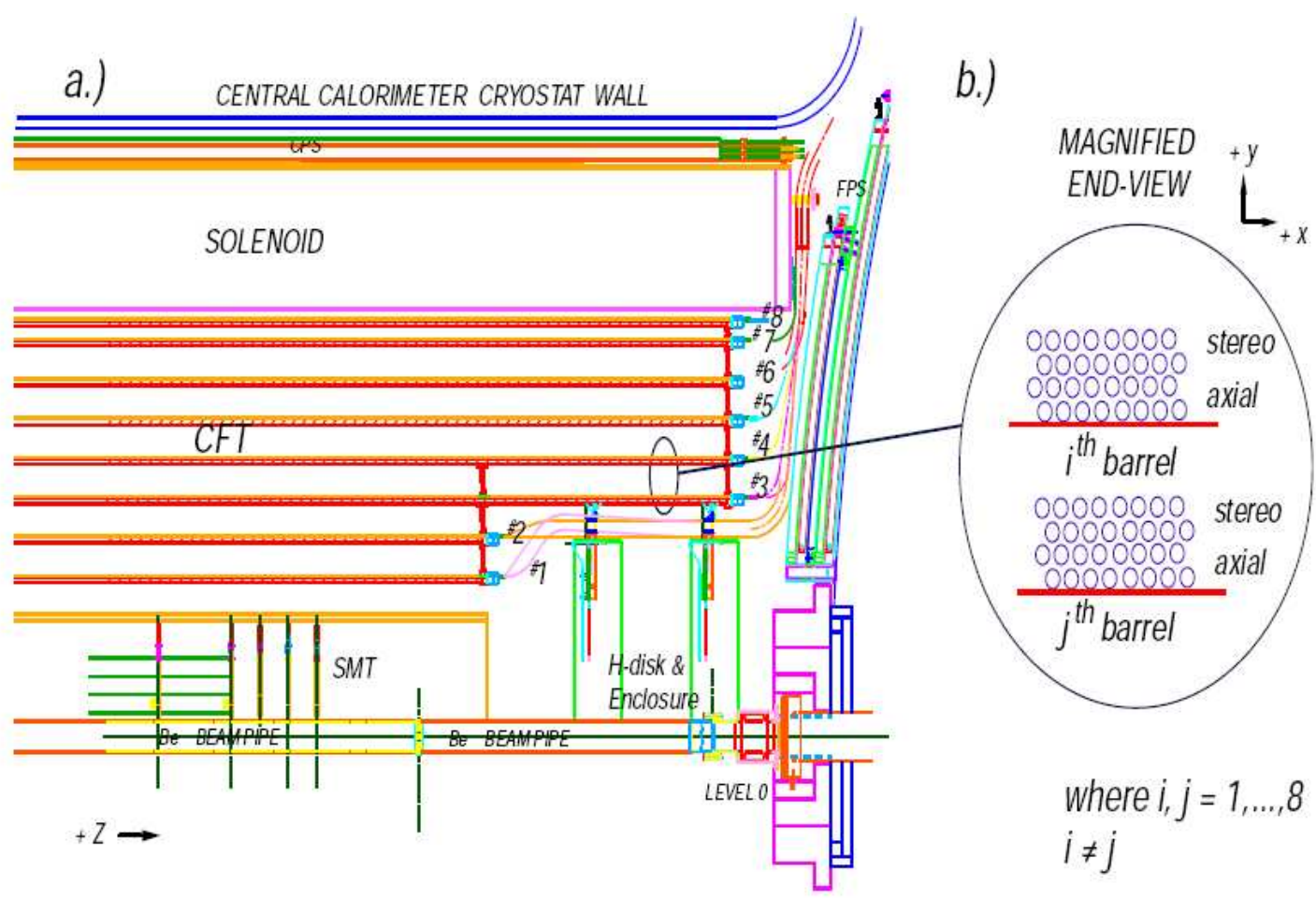

Figure 3.8: $r-z$ view of a quarter of the $\mathrm{D} \varnothing$ tracking system. The insert shows more details of the configuration of the CFT.

The light is only observed from one end of each scintillating fiber. The opposite end of the scintillating fibers is sputtered with an aluminum coating that provides a reflectivity of 85 to $90 \%$. The scintillating light is further propagated through clear fiber waveguides to visible light photon counters (VLPCs) located at the end of the waveguides. The VPLCs, situated outside of the detector acceptance, read out the signal and convert it to an electrical pulse. 


\section{Solenoid Magnet}

The transverse momenta of charged particles are determined from their curvature in the 2 Tesla magnetic field produced by the solenoid magnet [51]. The solenoid is a $2.73 \mathrm{~m}$ long superconducting magnet with a mean radius of $60 \mathrm{~cm}$. The uniformity of the field inside the tracking volume is better than $0.5 \%$ and the solenoid has a thickness of approximately 0.9 radiation lengths.

\section{Layer 0}

The extended running period of the Tevatron until 2009 made an upgrade of the existing DØ silicon tracker mandatory. First, plans were made to exchange the complete SMT detector [52]. After cancellation of this project at the end of 2003 due to budget constraints, it was immediately decided to construct a new inner silicon layer [53] which is closer to the beam line than the SMT. The so-called Layer 0 uses much of the existing infrastructure of the "old" SMT. The outer H-disks on each side were removed and their readout is now used for Layer 0 . This design was rather challenging because it had to fit in the existing SMT and had to be able to slide over the beam pipe for its installation. A photograph of Layer 0 is shown in Fig. 3.9. .
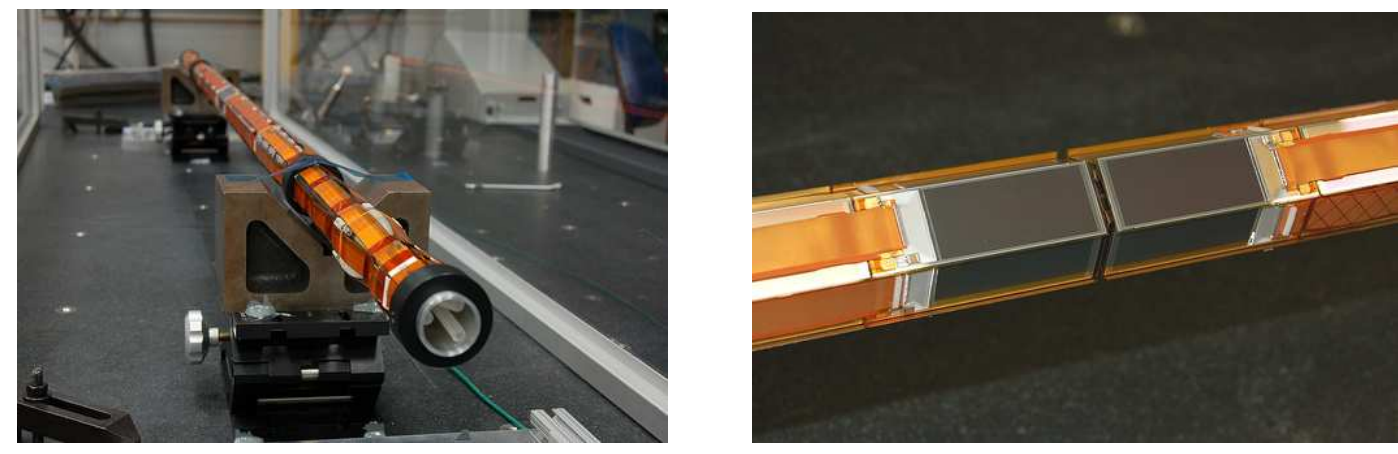

Figure 3.9: An overview of Layer 0 detector is shown on the left. The right photo shows the silicon sensors in the center of Layer 0

Layer 0 consists of 48 single-sided sensors with a $50 \mu \mathrm{m}$ readout pitch [54] and has an inner radius of $16 \mathrm{~mm}$ and and outer radius of $17.6 \mathrm{~mm}$. An individual Layer 0 module consists of a silicon sensor, a pair of analog cables with $91 \mu \mathrm{m}$ pitch stacked on top of each other with $45 \mu \mathrm{m}$ offset and a hybrid with two readout sensors.

During November 2005 a dedicated cosmics muon experiment has been set up in the SiDet laboratory to study the pedestals, noise and determination of the single track reconstruction [55]. The setup consisted of four layers of spare Layer 0 modules, precisely 
aligned with a cosmics muon trigger system. About 10000 cosmics muon events have been triggered, and about 4000 tracks have been fully reconstructed. The main results of this test are:

- We define hit finding efficiency as "number of track-pointed hits/the number of expected hits". The number of "Expected hits" is equal to the number of events that have a track. Tracks are formed with hits in the modules 1 and 4 and the efficiency is determined on hits in modules 2 and 3. The hit reconstruction efficiency is $96 \%$. Systematic errors were not taken into account. In the Monte Carlo simulation the corresponding efficency is found to be $99 \%$.

- The hit position resolution is defined as "measured position - projected position", where the "projected position" is the calculated position on module 2 or 3 of a track built out of hits in module 1 and 4 . The hit position resolution is about $11 \mu \mathrm{m}$ for incident muons, which is very well simulated in the Monte Carlo.

The installation and commissioning were during the shutdown in spring 2006. Layer 0 is important for the $\mathrm{D} \varnothing$ experiment in order to enhance the b-tagging capabilities due to an improved impact parameter resolution (see Fig. 3.10 and 3.11).

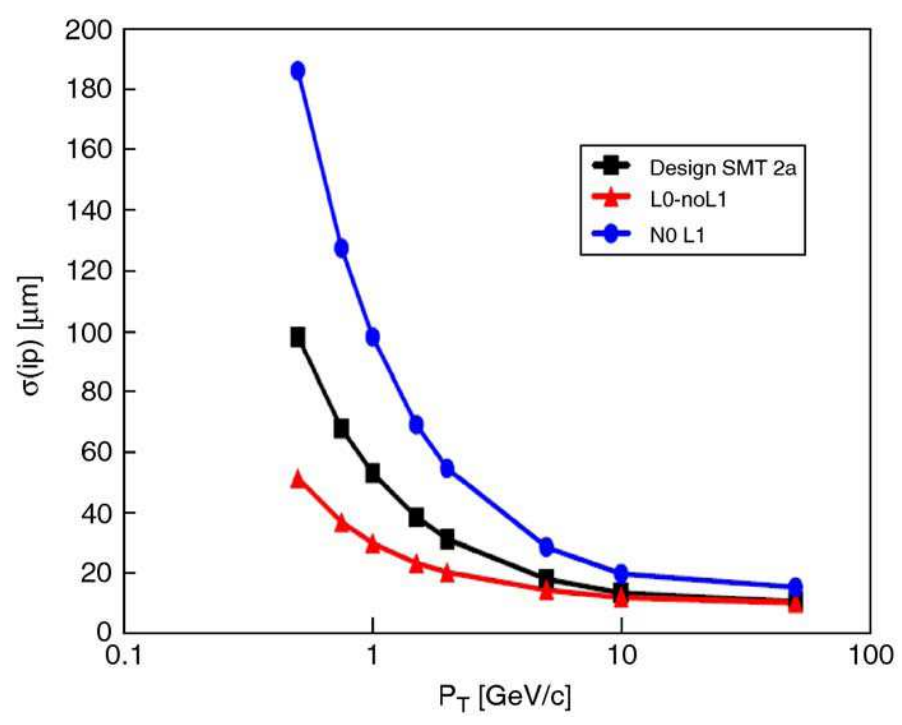

Figure 3.10: The three lines show simulations of the impact parameter resolution as a function of the transverse momentum of the tracks. The squares show the performance of the current Run IIa detector. The other two lines correspond to simulations with total loss of the innermost Layer-1 of the current Run IIa detector: one with the addition of Layer 0 (triangles) and one without (circles) [56]. 

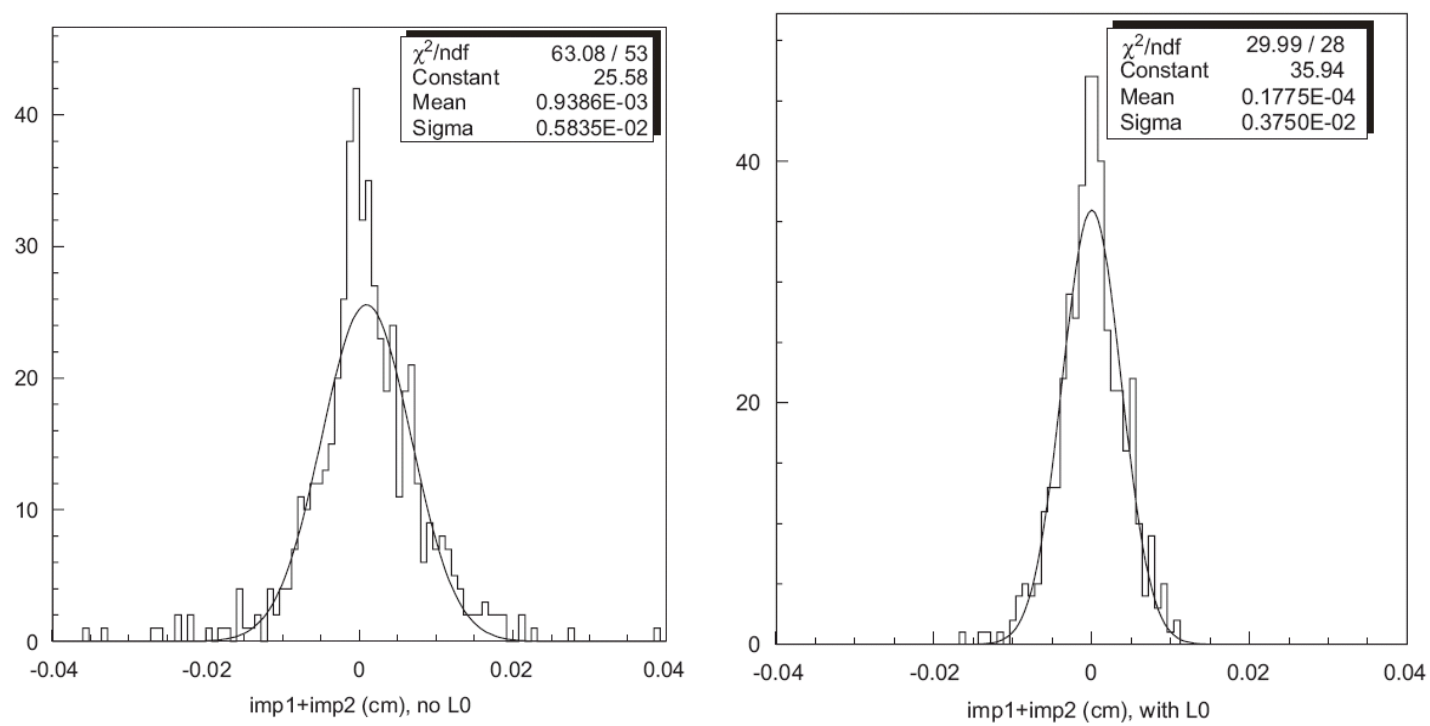

Figure 3.11: Impact parameter resolution for tracks without (left) and with (right) a hit in Layer 0 . The tracks are from cosmics muons with a $p_{t}<5 \mathrm{GeV} / \mathrm{c}$ [57].

\section{Preshower detectors}

The preshower detectors are situated just outside the solenoid and just before the calorimeter. They function as tracking devices as well as calorimeters and help in electron identification and triggering. They are used in the offline reconstruction to correct electromagnetic energy measurements of the calorimeter for losses in the solenoid, lead absorber, and in material such as cables and supports. The preshower system consists of the central (CPS) and the forward (FPS) preshower detectors.

The CPS are located in the $51 \mathrm{~mm}$ gap between the solenoid and the central calorimeter cryostat and cover a region of $|\eta|<1$.3. In front of the CPS a one radiation length thick $\mathrm{Pb}$ layer acts as a pre-radiator. The CPS are built of three concentric layers of scintillating strips: one inner axial layer and two outer stereo layers at an angle of $\pm 23^{\circ}$. A wavelength shifting fiber in the center of each element is used for the readout. The light is transmitted by clear waveguides and is read out by visible light photon counters (VPLCs) in a similar manner as in the fiber tracker.

The FPS are mounted on the faces of the end calorimeters and cover the region $1.5<|\eta|<2.5$. The design of the FPS is similar to that of the CPS. A layer of lead absorber is sandwiched between two scintillator planes in the region of $|\eta|>1.65$. In the smaller $\eta$ region electrons and photons are expected to shower as they pass through the solenoid, hence no additional material is used. Directly in front of the lead absorbers, there are two additional layers of scintillating pre-shower fibers which are known as the 
MIP layers. Photons do not leave a signature in the MIP and so the MIP layers can be used to distinguish muons, electrons and photons.

The preshower detectors are discussed in more detail in [58] and [59].

\subsubsection{Calorimeter system}

The calorimeters $[47,48,60]$ were designed to provide energy measurements for electrons, photons and jets and to assist in the particle identification. The system consists of three sampling calorimeters (electromagnetic, fine hadronic and coarse hadronic) and an intercryostat detector. An overview of the calorimeter system can be seen in Figure 3.12.

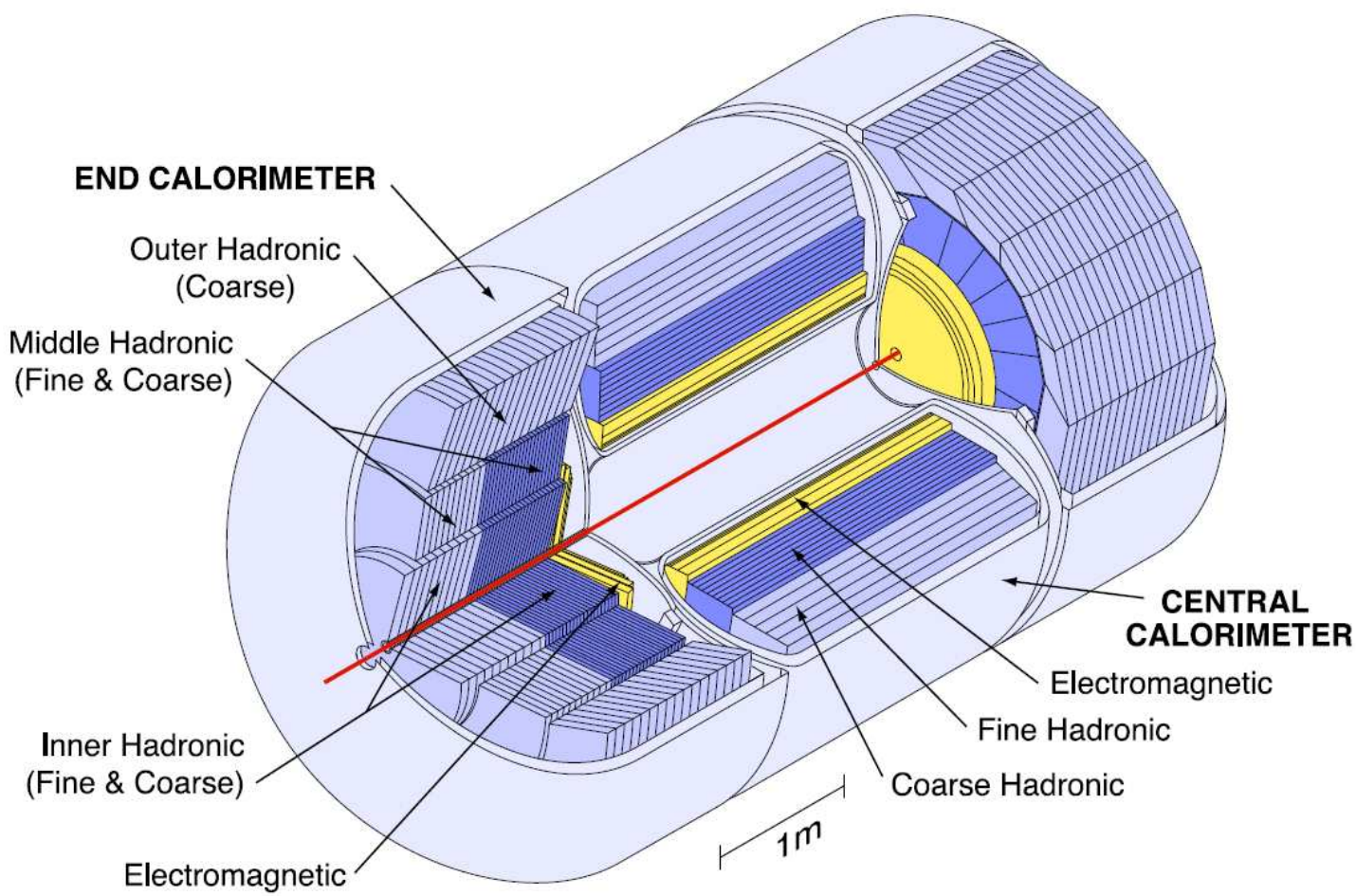

Figure 3.12: Isometric view of the central and two end calorimeters.

The central calorimeter (CC) covers $|\eta| \lesssim 1$ and the two end calorimeters, ECN (north) and ECS (south) extend the coverage to $|\eta| \approx 4$. Closest to the interaction region, there is the electromagnetic section followed by the fine and coarse hadronic sections. The active medium of the calorimeters is liquid argon. Each of the three calorimeters $(\mathrm{CC}, \mathrm{ECN}$ and ECS) is located within its own cryostat which maintains the detector temperature at approximately $90 \mathrm{~K}$. Different absorber materials are used in different locations. The electromagnetic section $(\mathrm{EM})$ uses thin plates $(3$ or $4 \mathrm{~mm})$ made from nearly pure depleted 
uranium. The fine hadronic sections are made from $6 \mathrm{~mm}$ thick uranium-niobium $(2 \%)$ alloy. In the $\mathrm{CC}$ the coarse hadronic modules contain relative thick $(46.5 \mathrm{~mm})$ plates of copper and in the EC there are stainless steel plates.

\subsubsection{Muon System}

The outermost detector subsystem is devoted to the detection and triggering of muons and to measure their momentum and charge. Due to the small interaction cross-sections of muons they pass all the material in the inner part of the detector while almost all electrons and hadrons are absorbed. The muon system is composed of sheets of scintillation pixels, proportional drift tubes (PDTs) and mini drift tubes (MDTs). These perform position measurements and time measurements, which also can be used to reject cosmic ray muons. A 1.8 Tesla iron toroid magnet provides the ability for a momentum measurement independent of the central tracking system. The muon system is divided into a central muon system with a coverage of $|\eta| \leq 1$ which uses PDTs for the position measurement and a forward muon system which covers $1<|\eta| \leq 2$ with MDTs. Both systems employ scintillating counters for triggering and vetoing of cosmic ray muons. Each system is split into three layers known as the A, B and C layers (see Fig. 3.13). Layer A is the innermost layer and lies just outside the calorimeter but inside the toroid magnet. The other two layers (B and $\mathrm{C}$ ) are positioned outside the toroid. In the region directly below the calorimeter, only partial coverage by muon detectors is possible since the support structure for the $\mathrm{D} \varnothing$ detector and readout electronics are located in this region. Fig. 3.13 and Fig. 3.14 show the layout of PDTs, MDTs and scintillators in an exploded view.

\section{Proportional Drift Tubes - PDTs}

The layout of the PDTs [61] can be seen in Fig. 3.13. They are rectangular $(\sim$ $2.8 \times 5.6 \mathrm{~m}^{2}$ ) gasfilled cells with anode wire at the center, cathode pads above and below the wire. Charged particles traversing this volume ionize the gas and the electrons from the ionization are collected and amplified by a wire in the center of the cell. The maximum drift time of the electrons in the PDTs is $500 \mathrm{~ns}$. The drift direction is parallel to the $z$-coordinate, with an expected drift distance resolution of approximately $1 \mathrm{~mm}$.

\section{Mini Drift Tubes - MDTs}

The MDTs [62] $\left(\sim 9.4 \times 9.4 \mathrm{~mm}^{2}\right)$ which cover the forward region (see Fig. 3.13) are similar to the PDTs but have shorter electron drift time $(<60 \mathrm{~ns}$, as opposed to almost $500 \mathrm{~ns}$ in the PDTs), and slightly better coordinate resolution of $\approx 0.7 \mathrm{~mm}$. 


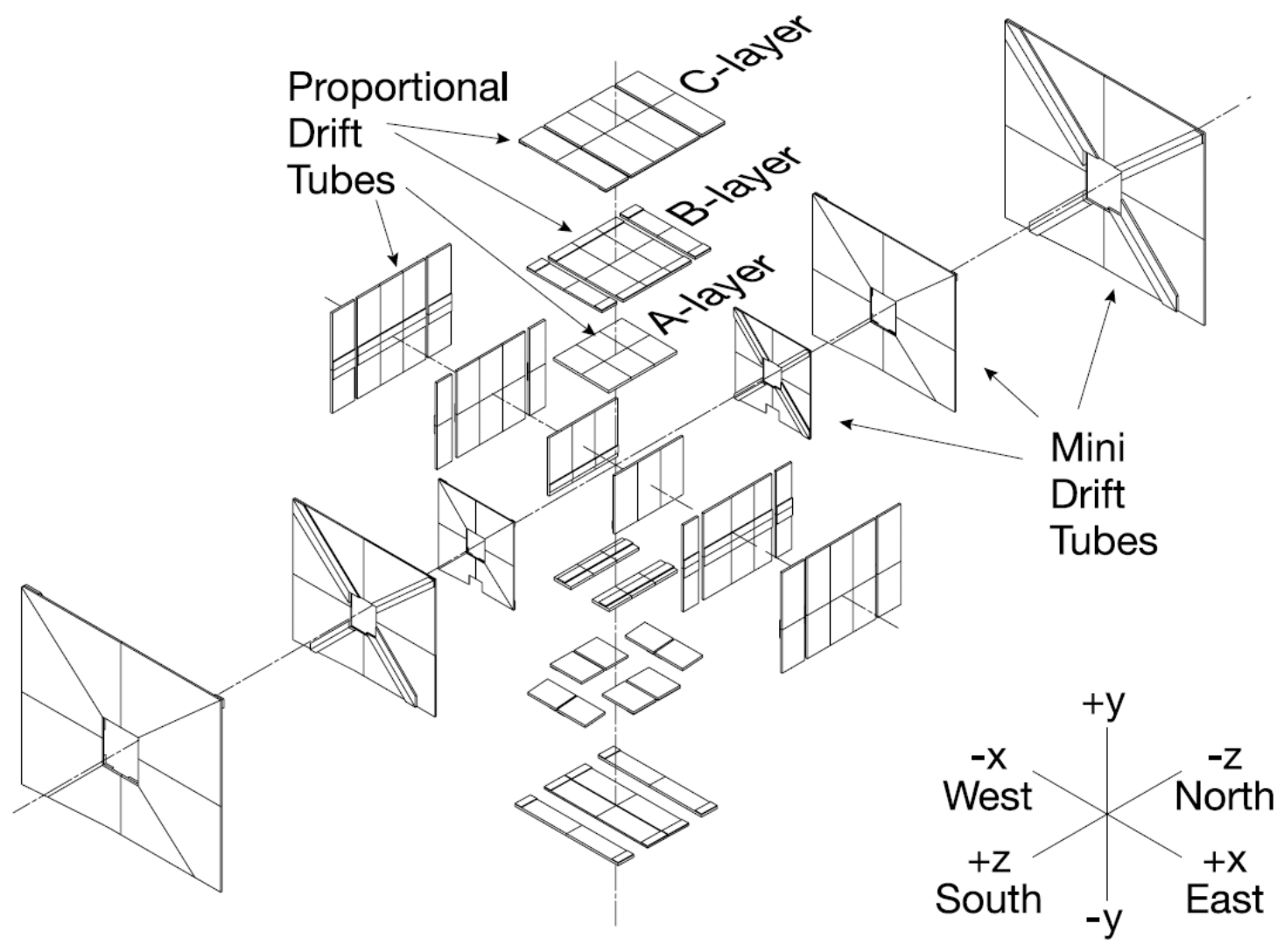

Figure 3.13: Exploded view of the muon wire chambers.

\section{Scintillation Counters}

Sheets of scintillating material serve to provide additional position measurements and are used for triggering, cosmic ray veto and track reconstruction. The scintillation light is collected by photomultipliers attached to one corner. Scintillating muon detectors are arranged in each layer except the B layer of the central muon system as shown in Fig. 3.14.

\section{Shielding}

In order to reduce background in the central and forward muon system, a shielding system consisting of layers of iron, polyethylene and lead in a steel structure is installed, around the beam pipe and the low beta quadrupole magnets. Iron is used as a hadronic and electromagnetic absorber, polyethylene is a good absorber for neutrons due to its high hydrogen content, and lead is used to absorb gamma rays. 


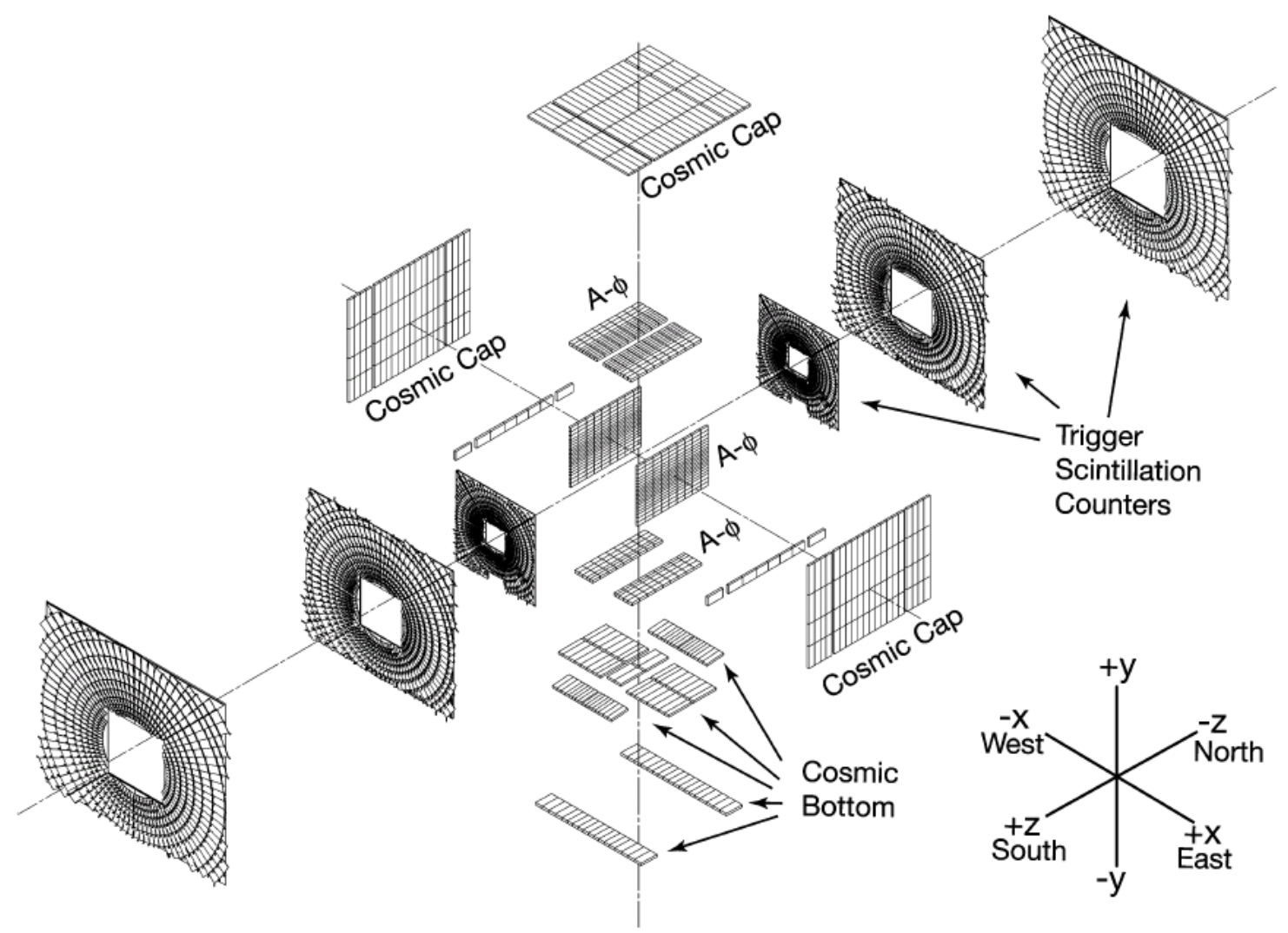

Figure 3.14: Exploded view of the muon scintillator counters.

\subsubsection{Luminosity System}

The primary purpose of the Luminosity Monitor (LM) [63] is to determine the Tevatron collider luminosity in the $\mathrm{D} \varnothing$ interaction region [64]. This requires a measurement of the rate of the inelastic $p \bar{p}$ interactions and is achieved by detecting the charged remnants of the proton and antiproton after the collision. The LM detector consists of two arrays of scintillation counters and is located in front of the end calorimeters. They occupy the radial region between the beam pipe and the forward preshower detector at a $z \sim \pm$ $140 \mathrm{~cm}$ as shown in Fig. 3.15. Each array consists of 24 wedges of scintillating material read out by photomultipliers and covers the pseudorapidity range $2.7<|\eta|<4.4$. 


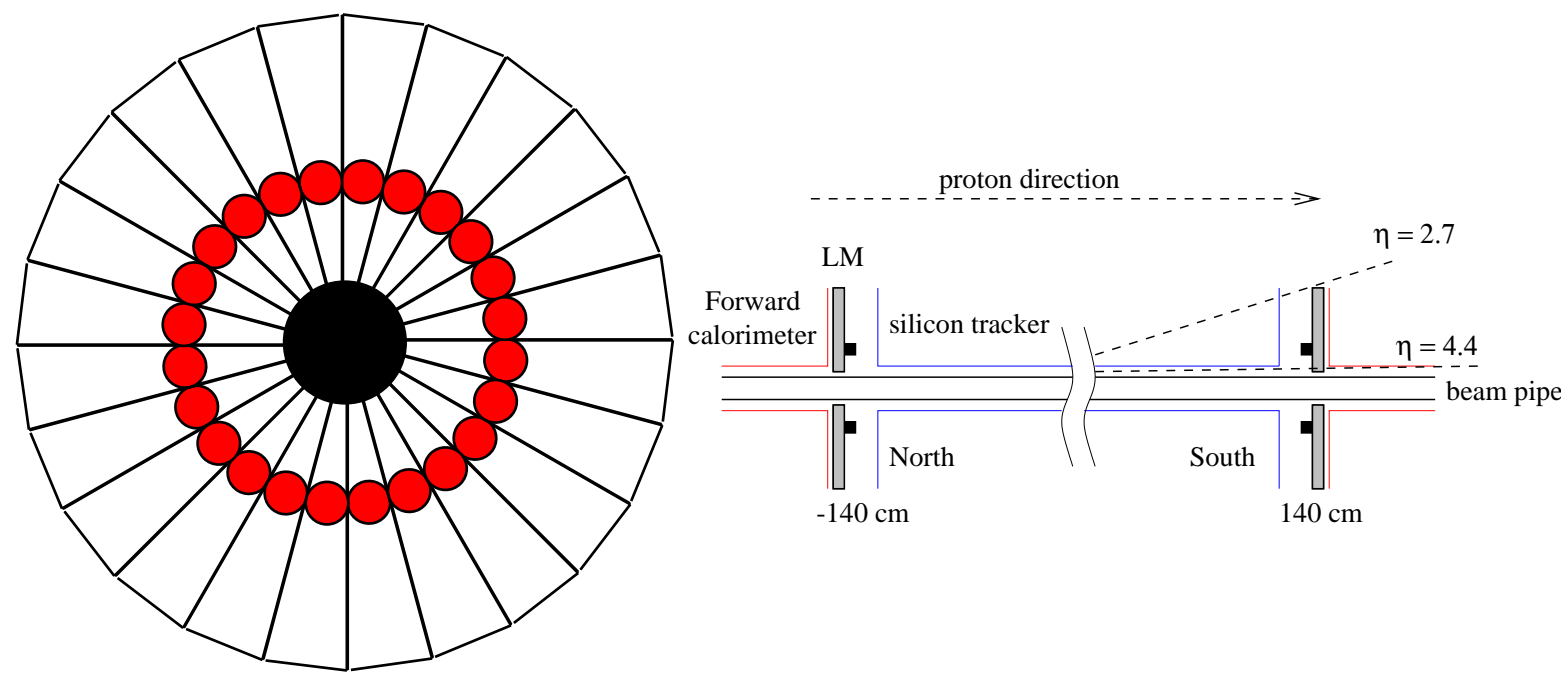

Figure 3.15: Luminosity Monitor layout. The $r-\phi$ view is shown on the left, the $r-z$ view of the two arrays is shown on the right.

\subsubsection{Trigger System}

Three distinct levels form the trigger system [65] with each succeeding level examining fewer events but in greater detail and with more complexity. An overview of the DØ trigger and data acquisition system is shown in Fig. 3.16.

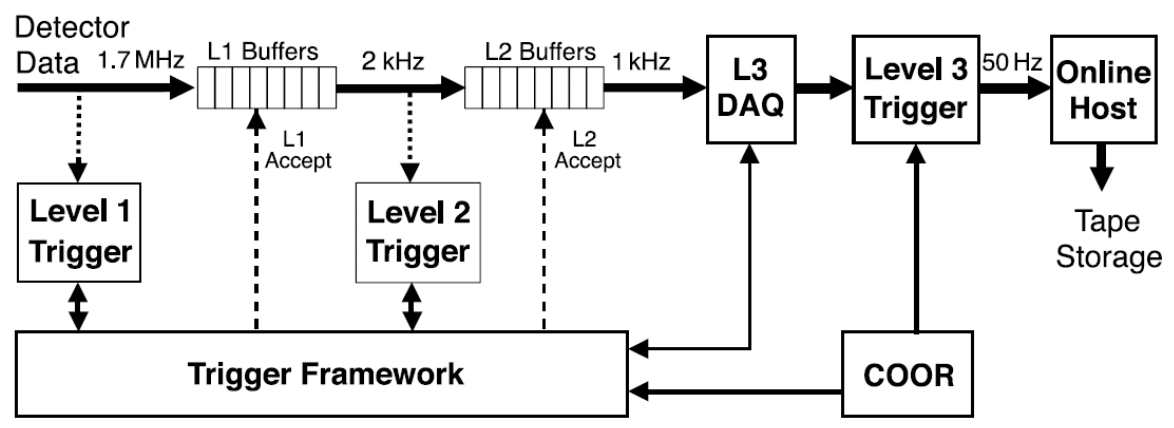

Figure 3.16: Overview of the integrated $\mathrm{D} \varnothing$ trigger and data acquisition system.

Collisions occur at a rate of $1.7 \mathrm{MHz}$. The first stage (Level 1 or L1) comprises a collection of hardware trigger elements that provide an accept rate of about $2 \mathrm{kHz}$. Events awaiting L1 trigger decisions, are buffered in a piplined and thus make minimal contribution to the deadtime. The trigger framework (TFW) gathers digital information from each 
of the specific L1 trigger devices and chooses if a particular event is to be accepted for further examination. In order to participate in the trigger decision the L1 trigger decision must arrive at the trigger framework in $4.2 \mu$ s or less.

The second stage (Level 2 or L2) [66] consists of two stages, preprocessors which are specific to each subdetector and a global processor which combines information from the different preprocessors to make a trigger decision. The L2 trigger system is the first one in the chain to look at event-wide variables to create objects like muons, electrons or jets. The L2 was designed to handle an input rate up to $10 \mathrm{kHz}$ with a maximum acceptance rate of $1 \mathrm{kHz}$. Events passing L2 are tagged for full read out and further analysis. A block diagram of the L1 and L2 system can bee seen in Fig. 3.17.

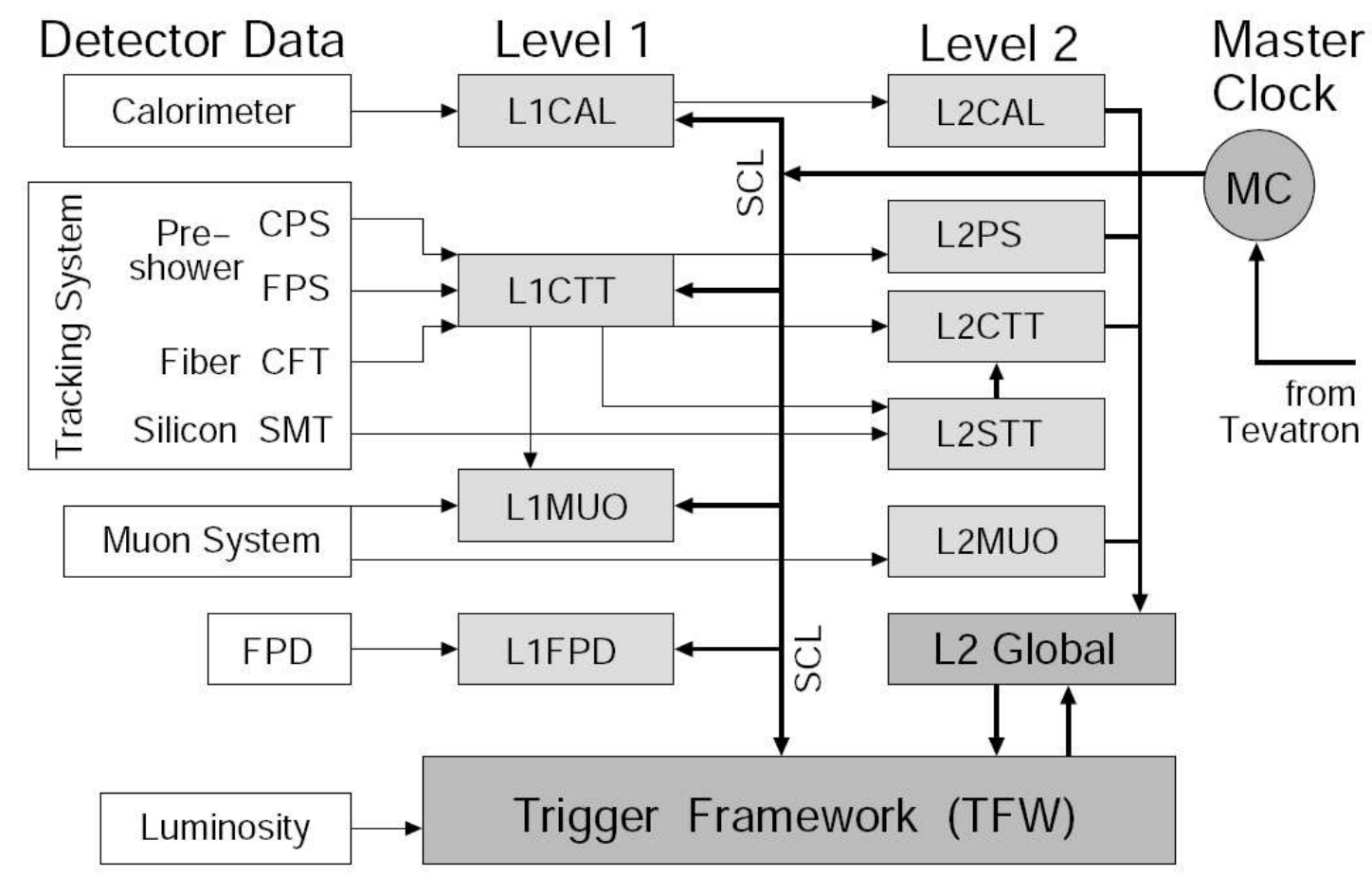

Figure 3.17: A block diagram of the L1 and L2 system.

Candidates passing L1 and L2 are sent to a farm of Level 3 (L3) [67] microprocessors where the rate is reduced to $50 \mathrm{~Hz}$. The L3 decisions are based on complete physics objects as well as the relations between such objects (such as rapidity or azimuthal angle separating physics objects or their invariant mass). Events also passing the L3 trigger are send to FERMILAB 's Feynman Computing Center, where the events are stored to tape for offline analysis. 


\section{Chapter 4}

\section{Event Reconstruction}

The raw data recorded from the $\mathrm{D} \varnothing$ Detector consists of digitized readout for each channel of each sub-detector such as pulse heights of collected charge in the calorimeter and silicon, light yields from the scintillators, hits in the tracking system, drift time from muons etc. The D $\varnothing$ reconstruction program D $\varnothing$ Reco [68] uses the raw data as input and decodes the detector hits, fits track trajectories and uses particle identification algorithms to create the particle objects like muons, electrons, jets and photons. The major steps of the D $\varnothing$ Reco are:

1. Decoding hit information: The digitized signals from the tracking detectors (SMT and CFT) are converted to spatial locations of the hits. The output of the calorimeter cells is decoded to energy deposits.

2. Tracking and Clustering: The hits from the tracking system are then combined to form tracks while the calorimeter energy deposits are grouped to form clusters.

3. Vertexing: With the help of various kinematic quantities the location of the $p \bar{p}$ interaction point (primary vertex) and decay vertices of long-lived particles (secondary vertices) can be found. The vertices are essential for the particle identification.

4. Particle identification: The tracking and calorimeter information is combined to form candidates for muons, electrons, photons, etc.

The reconstruction and particle identification algorithms used in this analysis ,such as muon identification, track and vertex reconstruction, are discussed in this chapter. 


\subsection{Track Reconstruction}

The reconstruction of particle trajectories is a first step in the event reconstruction. The trajectories of all charged particles are reconstructed from the energy they deposit in the tracking detectors i.e. in the SMT and the CFT. Two algorithms are used to find the tracks, the histogram track finding algorithm (HFT) [69] and the alternative algorithm (AA) [70]. The collection of track candidates from the AA and HTF methods are combined and duplicates are removed. .

\subsubsection{HFT Tracking Algorithm}

A charge particle will follow a helical path as it travels through the longitudinally oriented magnetic field of solenoid. The projection of this path on the $x \times y$ plane will form a circle of radius $\rho=\frac{q B}{p_{t}}$ and position $\phi$, where $q$ is the charge of the particle, $B$ is the magnitude of the magnetic field, $p_{t}$ is the transverse momentum of the track and $\phi$ is the angular direction of the particle at the distance of closest approach (DCA) to the beam spot. Since all points along the true path will have identical values for $\rho$ and $\phi$, it is possible to identify hits that belong to the same particle track by forming a histogram of the hits in the $\rho \times \phi$ coordinate space. By using the Hough transformation [71], a single hit in $x \times y$ coordinate space will correspond to a line of potential values in $\rho \times \phi$ plane. A similar transformation can be made between hits in the $r \times z$ plane to lines in the parameter space $z_{0} \times C$, where $z_{0}$ is the position of the track origin along the $z$ axis, and $C=\delta z / \delta r$ is the track inclination. The collection of lines from hits belonging to the same track will intersect at the true path coordinate in $\rho \times \phi$ and $z_{0} \times C$ planes and produce a peak in the resulting histograms. However, straightforward peak finding is not feasible. Instead, the histograms are cleaned up by removing bins with few entries, and then each bin in parameter space is considered to be a track template. Kalman filtering $[72,73]$ is used to select tracks from the templates.

\subsubsection{AA algorithm}

In the alternative algorithm, a pool of track candidates are created by using an extrapolation based on a cluster of 3 hits in the SMT. The second hit must be on a following layer within $|\delta \phi|<0.08$. The third, on a following layer, must be on a circle of radius greater than $30 \mathrm{~cm}$ and axial impact parameter with the beam spot of less than $2.5 \mathrm{~cm}$. The overall fit must have $\chi^{2}<16$. Each track is extrapolated to the next layer of the SMT or CFT and hits are added to the track hypothesis if the increase of $\chi^{2}$ is less than 16. If there are multiple hits in a given layer, they each become a new hypothesis. A certain number of misses (i.e., no hits) in layers are allowed to improve the efficiency. The pool of potential tracks are then sorted with precedence being given to the candidates with the greatest 
number of hits. For candidates with the same number of hits, precedence is given to those with the least number of missing layers. In the case of a double degeneracy, precedence is given to a candidate with the smallest $\chi^{2}$. To reduce false candidates a veto, based on the number of shared hits, is applied to candidates with a lower importance. To further reduce the number of fake tracks, primary vertices are determined using the accepted tracks, and every track that comes close to a vertex is given two additional hits in the ranking. Using this new weight, the tracks are resorted, and a new pool of tracks is determined. The same procedure is now used with the CFT hits, however in this case the track candidate is required to pass near one of the primary vertices to control the huge combinatorics.

\subsection{Muons}

First information of the muon systems, such as hits in the drift chambers, is used to make local track segments in each layer (A, B and C). Hits in the scintillator paddles are added to these segments. Segments consistent with a common trajectory are merged into a local muon track [74]. In order to form a global muon, the local track is combined with central tracks identified in the CFT and SMT. The matching is done using the error matrix propagation, which takes into account the magnetic field as well as Coulomb scattering and energy loss in the material of the solenoid and toroid magnets and the calorimeter. If the algorithm finds a match, the two tracks and two error matrices are combined to obtain the final muon track parameters.

The reconstructed muons are classified using type and quality. The type is given by the parameter nseg (see Table 4.1). A positive value indicates that local muon track is matched to a track in the central tracking system. A negative value indicates that the local muon could not be matched to a central track. The absolute value $|n s e g|=1,2$ or 3 gives information if the local muon is made up of hits only in layer A, only layer in B or C or in layer $\mathrm{A}$ and layers $\mathrm{B}$ or $\mathrm{C}$.

The muon quality can be LOOSE, MEDIUM or TIGHT. This criteria depends on the number of hits in the different layers.

- $n s e g=+1$ Loose/Medium muons

Muons with $n$ seg $=1$ are muons with an A segment matched with a central track. Such a muon is loose if it has:

- at least one scintillator hit

- at least two A layer wire hits

- $n s e g=+2$ Loose/Medium muons

Muons with $|n s e g|<3$ can only be loose or medium if they are matched to a central 
track. $n s e g=2$ muons are muons with a BC segment matched with a central track. Loose requires

- at least one BC scintillator hit

- at least two BC layer wire hits

- $|n s e g|=3$ Medium/Loose muons

When $|n s e g|=3$ muon candidate is medium if it has:

- at least one scintillator hit

- at least two A layer wire hits

- at least three BC layer wire hits

- a converged local fit $\left(\chi_{l o c}^{2}>0\right)$

The muon is defined loose if one of the above criteria fails.

- Tight muons

Only $\mid$ nseg $\mid=3$ muons can be tight. A muon is tight if it has:

- at least one scintillator hit

- at least two A layer wire hits

- at least one BC scintillator hit

- at least three BC layer wire hits

- a converged local fit $\left(\chi_{l o c}^{2}>0\right)$

A muon is Medium if it fulfills the above requirements and if its located in the bottom part of the detector (octant 5 and 6 with $|\eta|<1.6$ ).

A more detailed description of the muon quality can be found in [75].

\subsection{Vertex Reconstruction}

The algorithm for vertex reconstruction must be able to reconstruct the primary vertex and displaced vertices (secondary vertices) in an event with high accuracy. For details of the vertex reconstruction see $[76,77]$. 
Table 4.1: Criteria for the nseg variable

\begin{tabular}{l|c|c}
\hline \hline nseg & Muon Type & Central track matching algorithm \\
\hline \hline 3 & Central track + & $\begin{array}{c}\text { Muon to central if local } \\
\text { muon track fit converged. } \\
\text { local muon track } \\
\text { (A and BC layer) }\end{array}$ \\
\hline 2 & Central track + BC only & central to muon \\
\hline 1 & Central track + A only & central to muon \\
\hline 0 & Central track + muon hit & central to muon \\
\hline-1 & A segment only & no match \\
\hline-2 & BC segment only & no match \\
\hline-3 & local muon track (A+BC) & no match \\
\hline \hline
\end{tabular}

\section{Primary Vertex (PV)}

For the primary vertex determination an iterative method is used. The finding of the primary vertex starts from all selected tracks and the procedure is:

1. Fit a vertex from a set of tracks (total number $\left.N_{t r k}\right)$ and compute $\chi^{2}\left(N_{t r k}\right)$.

2. Each track is removed separately and a new $\chi^{2}\left(N_{t r k}-1\right)$ is calculated.

3. Select the track with the maximum difference $\Delta_{\max }=\chi^{2}\left(N_{t r k}\right)-\chi^{2}\left(N_{t r k}-1\right)$.

4. Exclude the track from the set if $\Delta_{\max }>\Delta_{\text {threshold }}$.

5. Repeat the procedure as long as there are tracks with $\Delta_{\max }>\Delta_{\text {threshold }}$.

A minimum of two tracks is required to fit a vertex candidate.

\section{Secondary Vertex (SV)}

Secondary vertices are due to the decay of long lived particles such as B hadrons. Reconstructing a SV is more difficult than a PV because there are fewer tracks associated to the SV. Tracks from the SV usually have a large impact parameter with respect to the PV what is shown in Fig. 4.1. The first step in the reconstruction of a SV is to make a list of all tracks that do not originate from the PV. The procedure of finding a SV is described as: 
1. Make a vertex out of two tracks not belonging to the PV or a previously find SV

2. Fit the vertex from the set of $N_{t r k}$ tracks $\left(N_{t r k}=2\right.$ for the first time) and compute $\chi^{2}\left(N_{t r k}\right)$.

3. Compute the new $\chi^{2}\left(N_{t r k}+1\right)$ obtained by adding each of the other tracks one by one.

4. Select the track with the smallest contribution $\Delta_{\min }=\chi^{2}\left(N_{t r k}+1\right)-\chi^{2}\left(N_{t r k}\right)$.

5. Add the good track to the set if $\Delta_{\min }<\Delta_{\text {threshold }}$.

6. Repeat the procedure from step 2 until there are no tracks fitting the requirements.

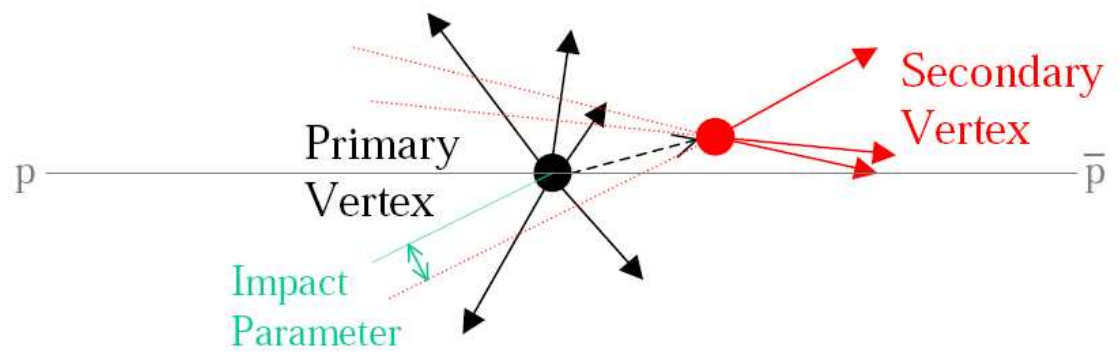

Figure 4.1: Primary and Secondary vertex. Tracks from SV have a in general a large impact parameter with respect to the PV 


\section{Chapter 5}

\section{Stability checks of the tracking alignment}

In this Chapter stability checks of the software alignment procedure of the $D \emptyset$ tracker are presented. The tracking system of the DØ detector consists of the SMT and the CFT detectors as described in section 3.2. The resolution of the SMT is about $10 \mu \mathrm{m}$ an the CTF has a resolution of about $100 \mu \mathrm{m}$. The structure of SMT is extremely complicated. The barrel is build of rectangular elements ("ladders"), and the disks of wedge-shaped elements ("wedges"). In contrast the structure of the CFT is relatively simple. This detector contains of eight cylindrical barrels of fibers directed along the beam line. Each barrel has an "axial layer", parallel to the beam axis and a "stereo layer" with an angle of $3^{\circ}$ with respect to the beam axis. The fibers in each layer are grouped into "ribbons". The number of ribbons varies from 10 in the first CFT layer to 28 in the last layer.

The alignment of the detector should be better than its intrinsic spatial resolution such that misalignments do not deteriorate the resolution of the track reconstruction. The positioning, or alignment, of the individual detector units is determined by using reconstructed tracks. When the presumed position of a given detector element is off from its true location, the offsets will manifest themselves as systematic anomalies in the residuals, i.e. the difference between the position of the measured hit and reconstructed trajectory. The distribution of residuals for a well aligned detector system should show a gaussian shape with zero mean and a standard deviation close to the intrinsic detector resolution.

The stability of the alignment algorithm was checked by changing the nominal positions of detector elements such as:

- Single CFT ribbon

- CFT layer

- SMT layer 
- All SMT elements

The modified configuration is then aligned and compared to the original aligned geometry. The procedure to check the stability of the alignment algorithm is depicted in Fig.5.1.

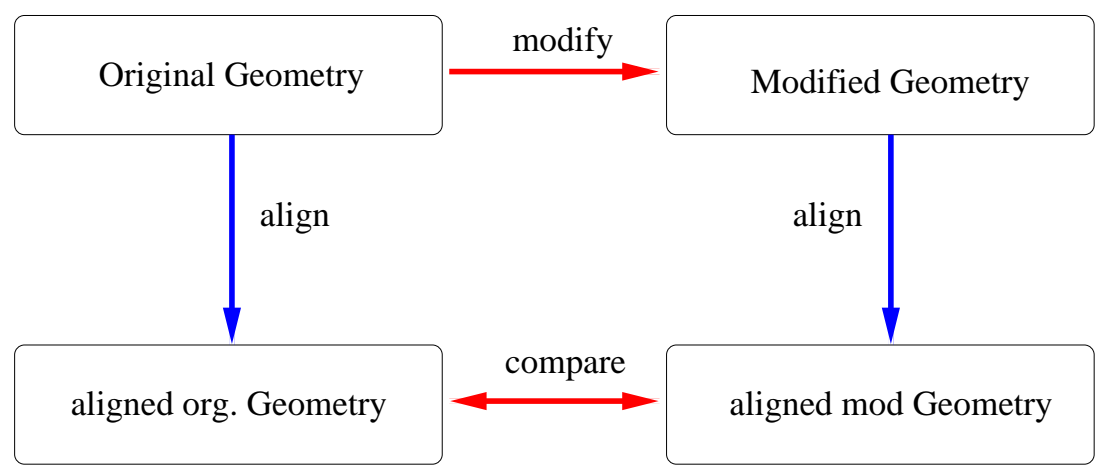

Figure 5.1: The procedure to check the stability of the alignment algorithm by comparing original and modified geometry after the alignment.

\subsection{Cosmics Data, reconstruction and geometry}

The cosmics muon data used for the alignment procedure was taken without magnetic field in the DØ Detector in November 2005. In total about 40000 cosmics muon events were reconstructed. The muons were reconstructed as a single straight track passing through the whole detector by matching two tracks, one from the upper half $(+x)$ and one from the lower half $(-x)$ of the detector. The position of each SMT ladder is described by three coordinates $x, y$ and $z$ of the center of gravity and the three Euler-angles of its orientation in space. The ribbons of the CFT are given by the $x$ and $y$ position of the corresponding circle which is approximating the ribbon.

\subsection{Alignment procedure}

The alignment procedure consists in determining the position and the rotation angles of the basic elements of the $D \varnothing$ tracking system. The basic element is a ladder or a wedge for the SMT, and a ribbon for the CFT detector. As a first step in the alignment, a large number of tracks passing through a given detector element are collected, and a residual for each track is determined. To avoid biases, the expected track position is determined excluding the detector element itself from the track fit (see Fig. 5.2). Any misalignment of the detector element results in the non-zero mean value of the residual distribution. 
Since many detector elements are included in the track measurement, the observed residuals in different elements are correlated. In this alignment procedure the shifts for each element are determined independently, and the correlation between the elements is taken into account by iterating the alignment until the resulting shifts of all detectors are below a predefined threshold. A detector element can be aligned if it has 10 or more hits otherwise the statistics is insufficient for alignment and the element is left at its nominal position. The data set is skimmed during the first alignment iteration using only good tracks which fit a certain quality criteria. Further iterations to align the detector are based on this selected tracks only. After 100 iterations the alignment procedure is stopped. The whole procedure is started again and due to some alignment more tracks pass the selection criteria and this helps to improve the alignment further. In the following the word CYCLES refers to a repeated alignment run with the whole data set.
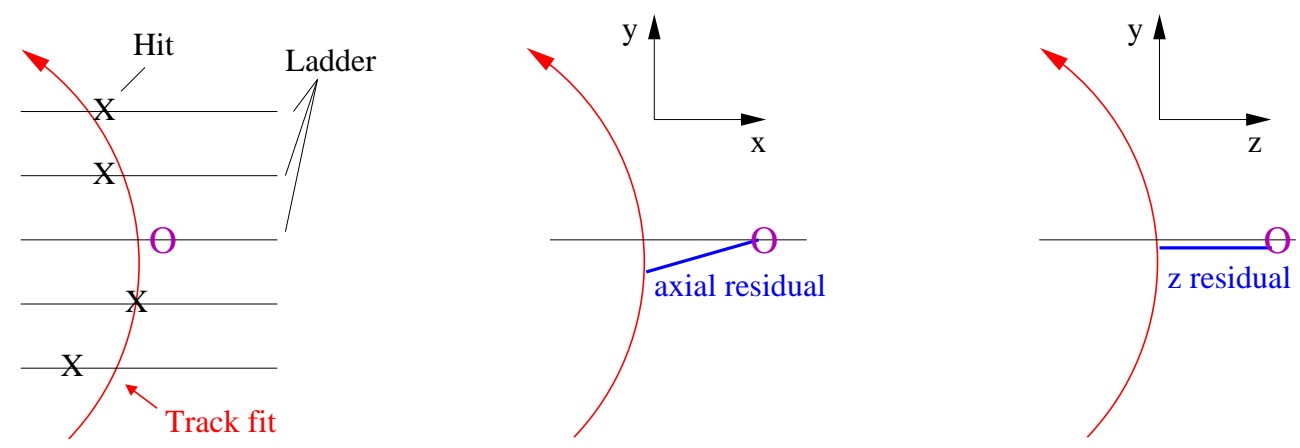

Figure 5.2: The hit "O" on the ladder is excluded for the track fit. The axial residual is calculated in the $(x, y)$-plane and the $\mathrm{z}$ residual on the $z$ axis.

\subsection{Different geometry modifications}

In this section different geometrical modifications are described and their impact based on various alignment cycles is given. The following modifications have been tested:

- Translational shifts in $x$ - or $y$-direction up to $1 \mathrm{~mm}$.

- Radial shifts: Both coordinates $x$ and $y$ of the detector element are modified to shift radially via $x_{\text {mod }}=x \cdot k$ and $y_{\text {mod }}=y \cdot k$ for $k=1 \%$.

- Telescoping shifts (only for SMT): The telescoping effect is described by a shift of the $z$ coordinate so that $z=z_{0}+c \cdot r$ where $r=\sqrt{\left(x_{0}^{2}+y_{0}^{2}\right)}$ and $c=1 \%$ or $0.5 \%$. The telescoping shift produces larger displacements for detector elements further away from the beam pipe. 
These modifications are applied to single elements or whole layers of the CFT or SMT or even to the whole SMT. The alignment precision is given by the standard deviation of a gaussian fit applied on the peak region of the residual distribution.

\subsubsection{Shift of SMT layer 3 and layer 6 - random shifts}

In the actual detector not all elements of a detector part are shifted coherently into the same direction with the same displacement. In this section a more realistic scenario is tested. All elements of the SMT barrels layer 3 and layer 6 are shifted randomly in the direction of $\pm x$ and $\pm y$ by about $200 \mu \mathrm{m}$.

\section{One cycle}

The Fig. 5.3 shows that after the alignment CFT elements are centered around zero for both the axial and radial distributions. Both distributions show small tails on the sides which is due to the random shift in both directions of $x$ and $y$. The axial and radial alignment precision for the CFT is around $19 \mu \mathrm{m}$.

The radial distribution of the SMT layer 3 and 6 is shown in Fig. 5.4. Most elements of both layer are between $\pm 50 \mu \mathrm{m}$. The outliers are identified as ladders with insufficient number of hits to be aligned. For all SMT barrel elements the axial and radial precision is of about 3-5 $\mu \mathrm{m}$ (see Fig. 5.5). The tails in both distribution are a result of the elements from layer 3 and layer 6.
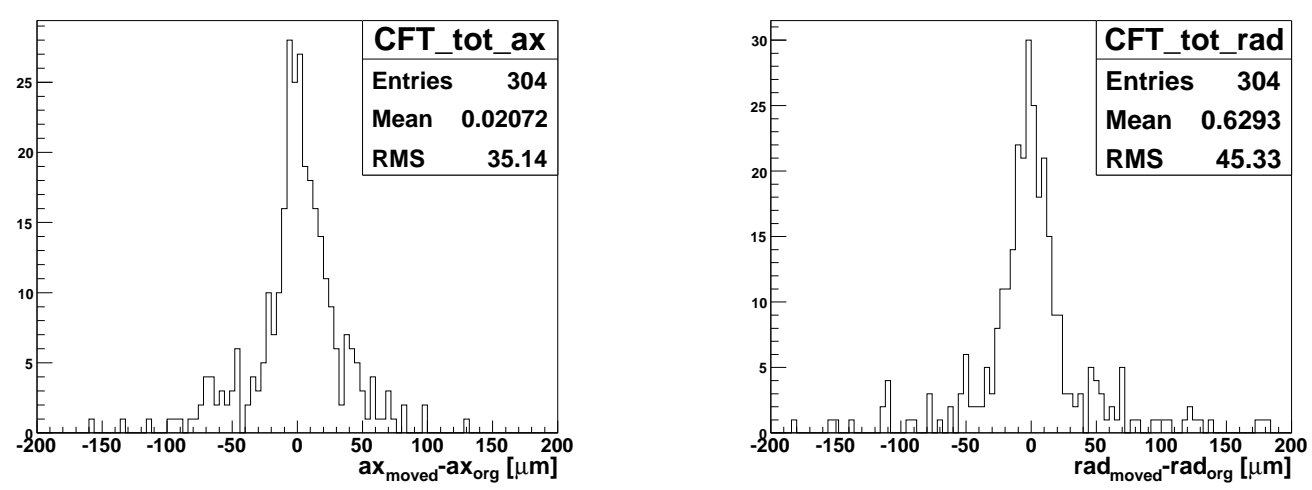

Figure 5.3: The axial- and radial-difference between the modified and the original aligned geometry for all CFT elements for random shifts of SMT layer 3 and 6. 

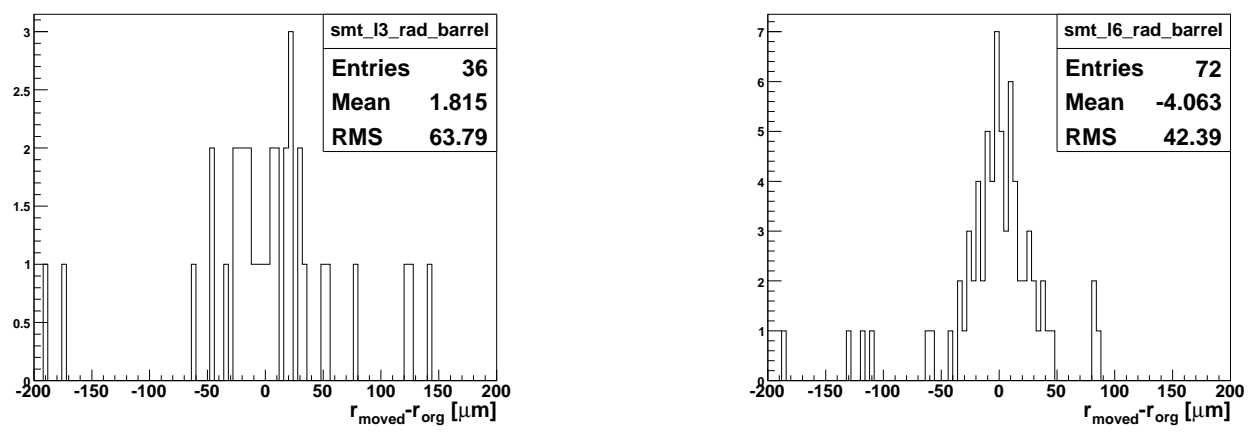

Figure 5.4: The radial-difference between the modified and the original SMT layer 3 (left) and SMT layer 6 (right) for random shifts.
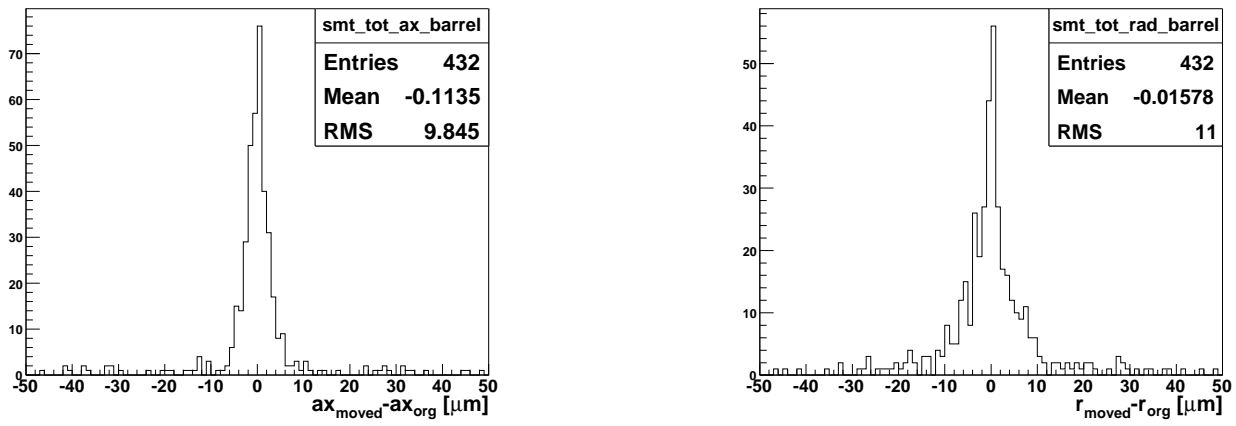

Figure 5.5: The radial-difference between the modified and the original aligned geometry for all SMT barrel elements, for random shifts of SMT layer 3 and 6.

\section{Four cycles}

To check if the alignment improves more cycles have been done. After the fourth cycle the alignment does not improve further. In the following the alignment after four cycles is presented.

Compared to Fig. 5.3, Fig.5.6 shows a clear improvement. The axial and radial alignment precision of the CFT improved to about $10 \mu \mathrm{m}$. The tails also disappeared for both distributions. Elements with a sufficient number of hits are well aligned and moved to the same position as in the aligned original geometry. The improvement of the alignment of the modified layers can be seen in Fig .5.7 compared to Fig 5.4. For the SMT barrel, the small tails in the axial and radial distributions due to layer 3 and layer 6 disappeared (see Fig. 5.8). The precision for the whole SMT barrel does not improve if cycles are repeated and stays at $3 \mu \mathrm{m}$. The alignment procedure works fine for random shifts of the SMT layers. 

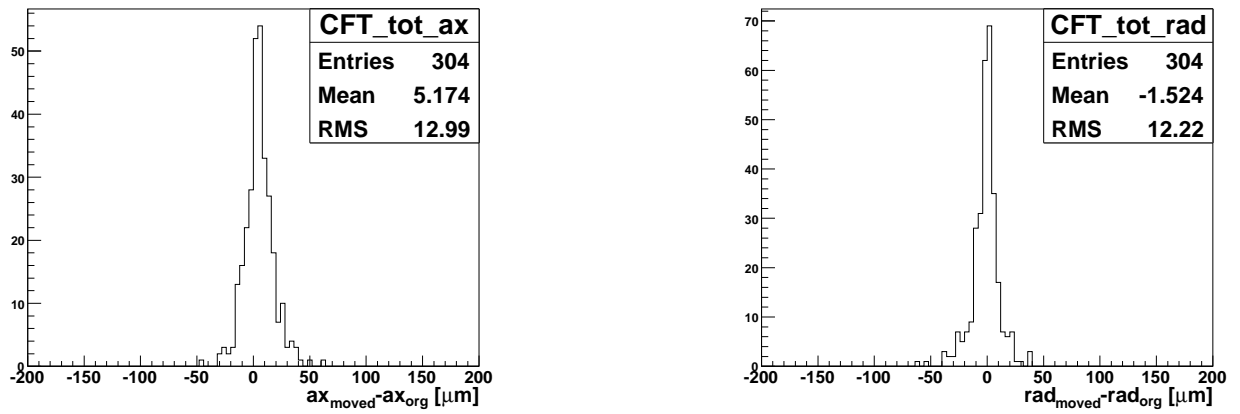

Figure 5.6: The axial- and radial-difference between the modified and the original aligned geometry after 4 cycles for all CFT elements for random shifts of SMT layer 3 and 6.
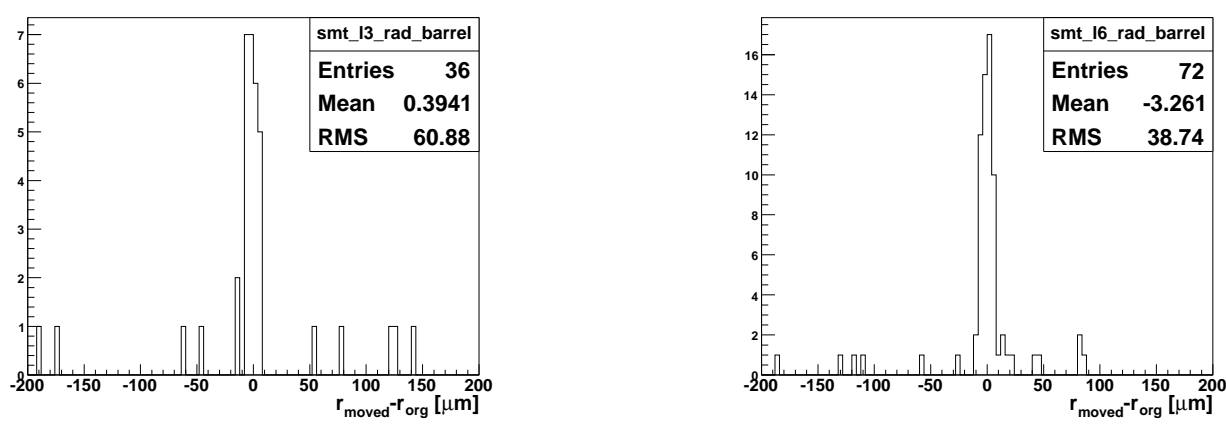

Figure 5.7: The radial-difference between the modified and the original SMT layer 3 (left) and SMT layer 6 (right) for random shifts after 4 cycles.
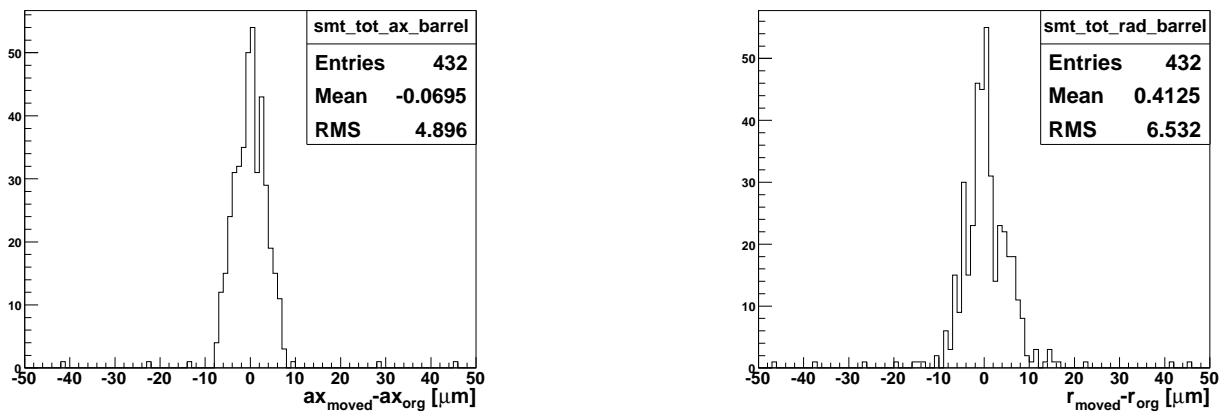

Figure 5.8: The radial-difference between the modified and the original aligned geometry after 4 cycles for all SMT barrel elements, for random shifts of SMT layer 3 and 6. 


\subsection{Summary and Conclusion}

From the various alignment studies using different modification of the initial geometry a final systematic uncertainty of the alignment was determined. For the CFT detector a systematic uncertainty on the position determination due to the alignment procedure of about $25 \mu \mathrm{m}$ (axial and radial) was found. For the SMT detector the systematic uncertainty for the axial precision is of the order of $5 \mu \mathrm{m}$, for the radial precision the order of about $7 \mu \mathrm{m}$ and for $z$ order of $15 \mu \mathrm{m}$. Table 5.1, an overview over the results of all different studies that were done in order to check the alignment stability is given.

Shifts of single elements do not influence the alignment and the shifted elements are well aligned after one cycle. Also modifications of whole layers of the CFT or SMT are well corrected after a few cycles $(<5)$. The radial shift of the whole SMT barrel resolves after more cycles (about 40). The telescoping effect is corrected by the alignment although there is a systematic shift if the CFT is not fixed. Also random shifts of different SMT ladders are well corrected by the alignment. The current alignment procedure of $\mathrm{D} \varnothing$ corrects various misalignments with a good precision. 


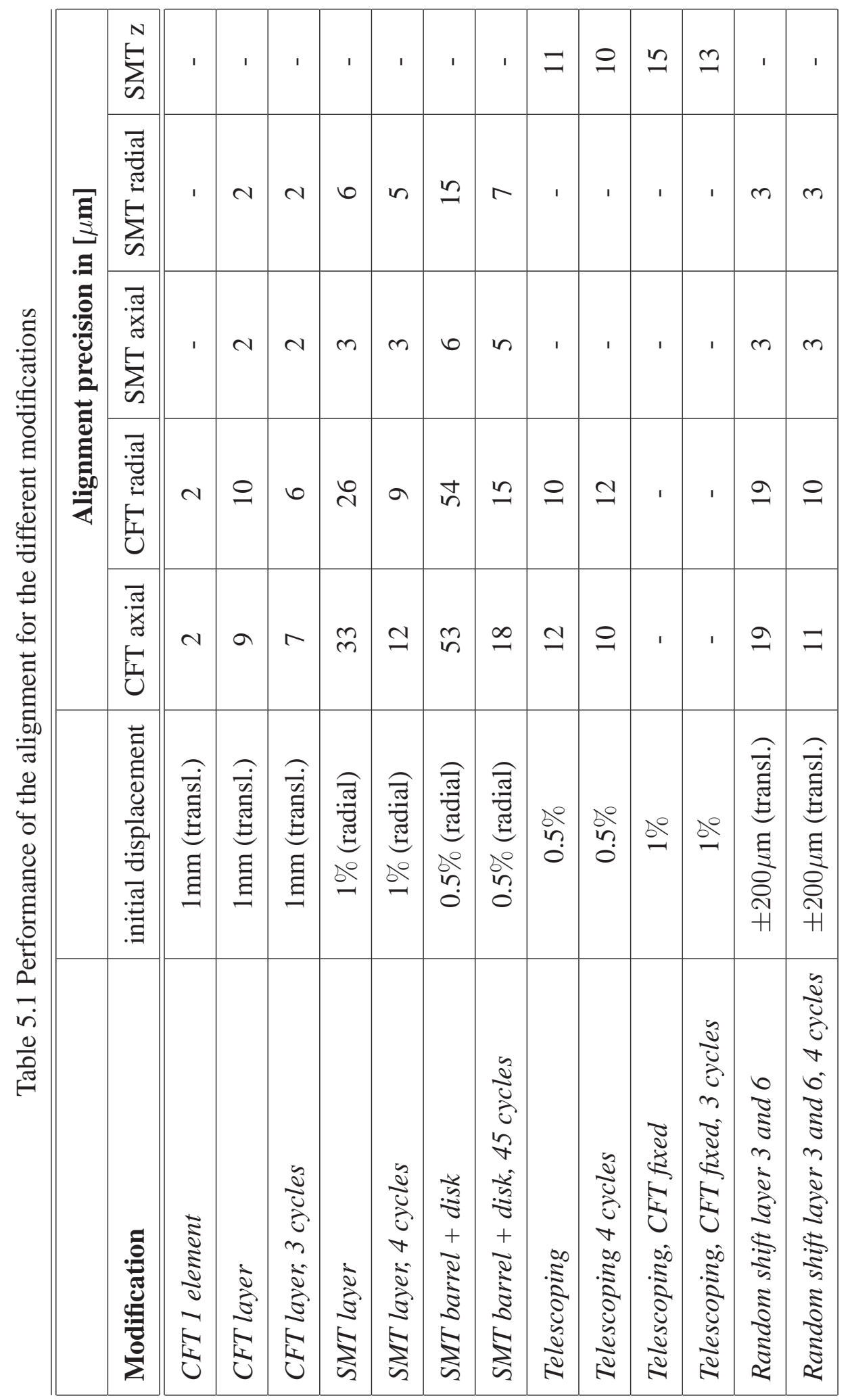




\section{Chapter 6}

\section{Multivariate Analysis Method}

The aim of multivariate analysis methods is to maximize background suppression while keeping the signal efficiency as high as possible. Traditional Simple-Cut techniques are straightforward but often have a poor performance. The main advantage of the multivariate analysis methods is that they make use of non-linear combinations of several variables which usually leads to an enhanced performance in seperating signal from background events. There are different approaches like Artificial Neural Networks (ANN) [78], Boosted Decision Trees (BDT) [79, 80, 81] and many others. In the presented analysis the BDT method is used and described in the following sections.

\subsection{Decision tree}

The BDT method is based on a decision tree [82] which is a sequence of binary splits of the data. At the beginning there is the root node which contains all signal and background events (see Fig. 6.1). Then the cut value on that variable with the best separation power is taken to split the data into signal and background. After the splitting there are two nodes (branches). This process is repeated on these new branches and continued until a given number of final nodes (leaves) is obtained, until a node has to few events or until all leaves are pure. We assume that the events are weighted with each event having weight $W_{i}$. For unweighted events $W_{i}$ is equal 1 . The purity $\mathcal{P}$ of the data in the node is calculated by:

$$
\mathcal{P}=\frac{\sum_{s} W_{s}}{\sum_{s} W_{s}+\sum_{b} W_{b}}
$$

where $\sum_{s}$ is the sum over signal events and $\sum_{b}$ is the sum over background events. For a leaf with only signal $(\mathcal{P}=1)$ or only background $(\mathcal{P}=0)$ events the value $\mathcal{P}(1-\mathcal{P})$ is 0 . In 


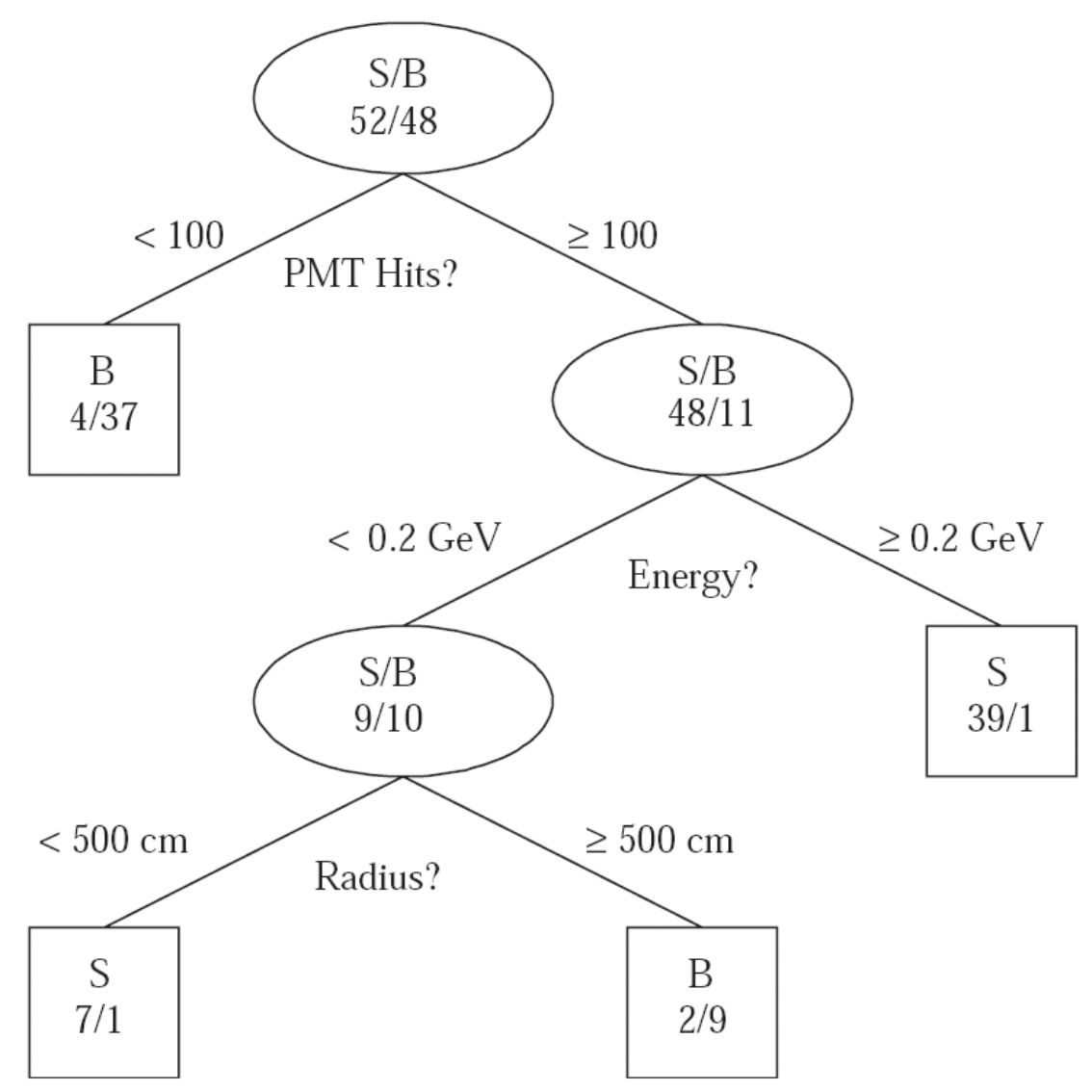

Figure 6.1: An example of a decision tree where the squares are the signal or background leaves [79].

order to define the quality of the separation between signal and background the Gini-value is used. It reaches its minimum (zero) when all events are signal or background like.

$$
\text { Gini }=\left(\sum_{i=0}^{n} W_{i}\right) \mathcal{P}(1-\mathcal{P})
$$

where $n$ is the number of events in that branch. In order to optimize the cut value on a variable the value of the following expression has to be minimized

$$
\text { Gini }_{\text {left son }}+\text { Gini }_{\text {right son }}
$$

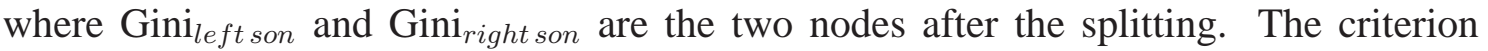
described in equation 6.3 gives the value for the best branch split. The increase in quality when a node $\left(\right.$ Gini $\left._{\text {father }}\right)$ is split into two branches $\left(\right.$ Gini $_{\text {right son }}$, Gini left son $_{\text {) }}$ ) is given by:

$$
\text { Gini }_{\text {father }}-\text { Gini }_{\text {left son }}-\text { Gini }_{\text {right son }}
$$


At the end, if a leaf has a purity greater than a given value $p$ it is called signal leaf or background leaf. To classify an event as signal or background it is checked if the event belongs to a signal leaf or a background leaf. The resulting tree is called a decision tree.

\subsection{Boosted Decision Tree}

The difference of a BDT to a standard decision tree is the boosting algorithm. A BDT learns from its mistakes. At the beginning all events are unweighted and a first tree is built. If a signal event ends up on a background leaf or a background event ends up on a signal leaf, then the weight of this event is increased (boosted). A second tree is then built, taking into account the new weights. The whole procedure can be repeated up to several 100 times.

The next step is to assign a score to an event. If an event is found on a background leaf it gets the score of -1 and if it is on a signal leaf the assigned score is +1 . At the end the sum of all scores is the final score of the event. Events with high scores are most likely signal events and events with low scores tend to be background like. This method of classification is very efficient and robust.

There are different methods for the boosting. One of the commonly used algorithms is AdaBoost [83] which also was used in this analysis.

\subsubsection{AdaBoost-Algorithm}

A sample of total $N$ events is taken and $N_{\text {trees }}$ trees are generated and the weight of each event is initially taken as $w_{i}=1 / N$. Furthermore:

- $m$ is the index of an individual tree

- $x_{i}$ : set of variables for the $i$-th event

- $y_{i}=1$ if the event is a signal event else $y_{i}=0$

- $w_{i}$ : the weight for each event

- $T_{m}\left(x_{i}\right)=1$ if the $i$-th event belongs to a signal leaf of the $m$-th tree, else $T_{m}\left(x_{i}\right)=0$

- $I_{i}=1$ if an event is misclassified $\left(y_{i} \neq T_{m}\left(x_{i}\right)\right)$, else $I_{i}=0$ if an event is classified correctly $\left(y_{i}=T_{m}\left(x_{i}\right)\right)$ 
For the $m$-th tree a misclassification rate $\mathrm{err}$ and a boost weight $\alpha_{m}$ can be calculated.

$$
\begin{gathered}
e r r_{m}=\frac{\sum_{i=1}^{N} w_{i} I_{i}}{\sum_{i=1}^{N} w_{i}} . \\
\alpha_{m}=\beta \times \ln \left(\frac{1-e r r_{m}}{e r r_{m}}\right)
\end{gathered}
$$

where $\beta=1$ in the standard AdaBoost method. Misclassified events are boosted by this factor, by changing the weight of each event to

$$
w_{i} \rightarrow w_{i} \times e^{\alpha_{m} I_{i}}
$$

The entire event sample is then renormalized to keep the total number of events (sum of all weights) in a tree constant $w_{i} \rightarrow w_{i} / \sum_{i} w_{i}$. The final score for an event is then given by:

$$
T(x)=\sum_{m=1}^{N_{\text {trees }}} \alpha_{m} T_{m}\left(x_{i}\right)
$$

A schematic overview how to get the final score of an event be seen in Fig. 6.2.

\subsection{Toolkit for Multivariate Analysis}

TMVA (Toolkit for Multivariate Analysis) [84] is a package integrated in ROOT [85] for processing and parallel evaluation of various multivariate classification techniques. The usage if this tool requires a sample of signal events and a sample of background events. The two samples are splitted into a training and a test sample, using the random splitting tool of the TMVA package. After training with the training sample, the obtained selection is tested on the independent test sample to estimate the accuracy of the procedure. It is important to have two independent samples for training and testing otherwise the result would be biased.

\subsection{Optimization}

After the evaluation of the BDT the optimal cut value on the BDT response, which is a combination of all cut variables has to be found. There are different approaches for the optimization to enhance the signal (S) over the background (B):

1. $S / \sqrt{B}$ 


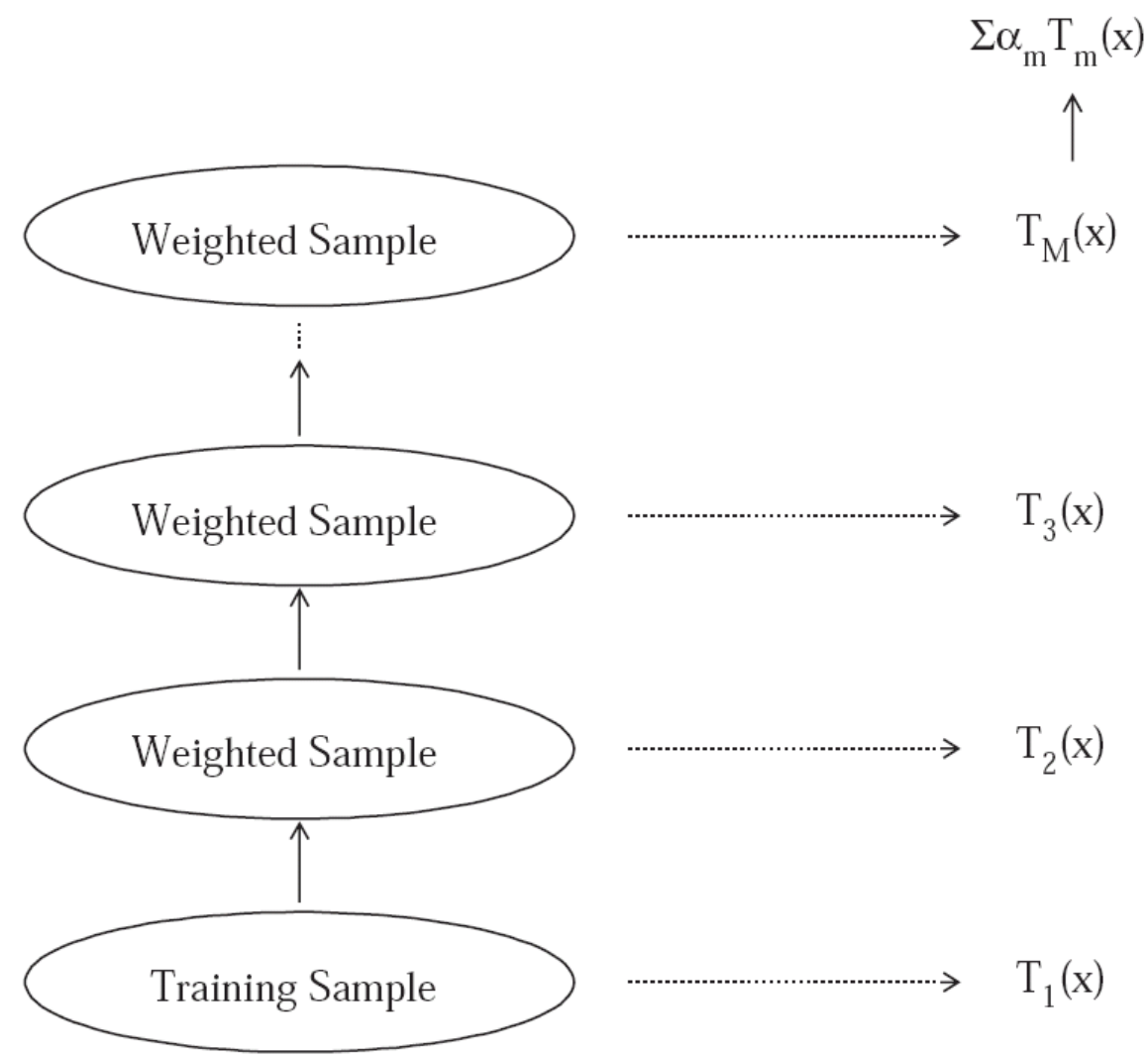

Figure 6.2: An overview of getting the final score of an event. [80].

2. $S / \sqrt{S+B}$

Maximization of these expression could lead to really low efficiencies and breaks down when the signal and backround tends to be 0 . An other approach is given by G. Punzi [86]. In an experiment a theory $H_{0}$ is the current best theory and as a result of the experiment we wish to confirm or disprove the theory $H_{0}$ in favor of a new theory $H_{m}$. In a counting experiment with presence of background there is the discrete observable $n$, the number of observed events, which are Poisson-distributed with mean of expected number of background events $B$ and a possible contribution of signal events $S_{m}$

$$
\begin{gathered}
p\left(n \mid H_{0}\right)=e^{-B} B^{n} / n ! \\
p\left(n \mid H_{m}\right)=e^{-B-S_{m}}\left(B+S_{m}\right)^{n} / n !
\end{gathered}
$$

To completely define the test a desired significance level $\alpha$ and CL has to be chosen and this leads to the constraint $S_{m}>S_{\min }$ (see Fig. 6.3). 


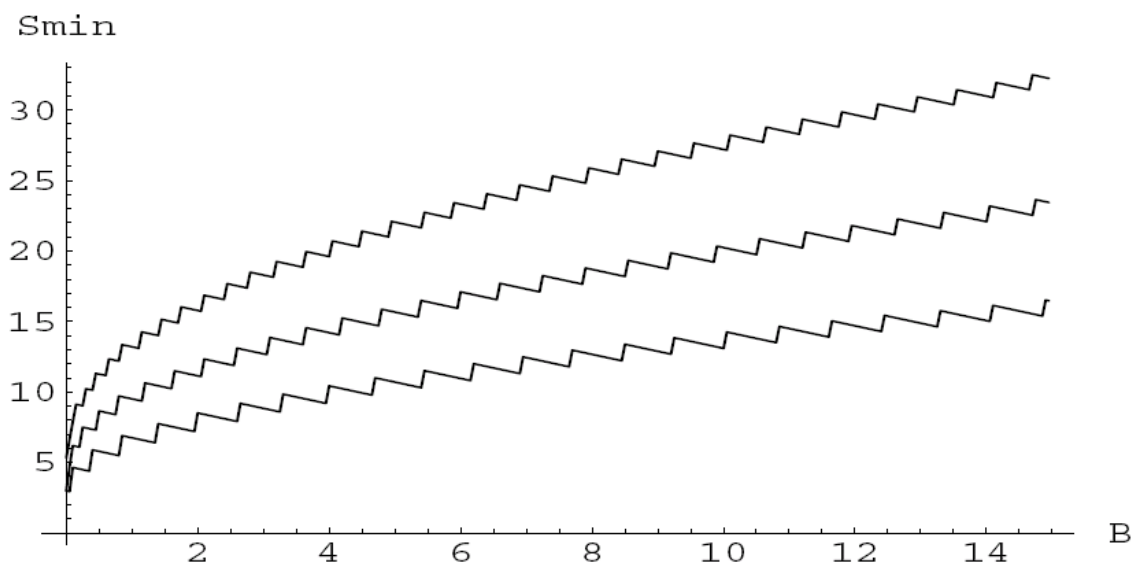

Figure 6.3: The lower limit of the sensitivity region $S_{\min }$ for a search experiment with (significance, CL) respectively of (95\%,95\%), $(3 \sigma, 95 \%)$ and $(5 \sigma, 90 \%)$ [86].

With a Gaussian approximation of the Poisson a simple result can be obtained.

$$
S_{\text {min }}=a \sqrt{B}+b \sqrt{B+S_{\text {min }}}
$$

where $a$ and $b$ are the number of standard deviations corresponding to one-sided Gaussian tests. Solving Equation 6.11 for $S_{\min }$ :

$$
S_{\text {min }}=\frac{b^{2}}{2}+a \sqrt{B}+\frac{b}{2} \sqrt{b^{2}+4 a \sqrt{B}+4 B}
$$

Equation 6.12 is valid for one specific set of data selection criteria but can be modified to a general case where $B$ and $S_{m}$ depend on the cuts $t$.

$$
S_{\min }(t)=\frac{b^{2}}{2}+a \sqrt{B(t)}+\frac{b}{2} \sqrt{b^{2}+4 a \sqrt{B(t)}+4 B(t)}
$$

Further the signal yield $S_{m}$ can be written as

$$
S_{m}(t)=\epsilon(t) \cdot \mathcal{L} \cdot \sigma_{m}
$$

where $\epsilon$ is the efficiency at a chosen cut value, $\mathcal{L}$ is the integrated luminosity and $\sigma_{m}$ is the cross section of the process being searched. Equation 6.14 can now be inverted to find the minimum "detectable" cross section.

$$
\sigma_{\text {min }}=\frac{\frac{b^{2}}{2}+a \sqrt{B(t)}+\frac{b}{2} \sqrt{b^{2}+4 a \sqrt{B(t)}+4 B(t)}}{\epsilon(t) \cdot \mathcal{L}}
$$


The maximum sensitivity is attained when $\sigma_{m}$ is smallest. This means that the Equation:

$$
\frac{\epsilon(t)}{\frac{b^{2}}{2}+a \sqrt{B(t)}+\frac{b}{2} \sqrt{b^{2}+4 a \sqrt{B(t)}+4 B(t)}}
$$

has to be maximized. By choosing $a=b$ the expression 6.16 becomes even simpler

$$
\frac{\epsilon(t)}{a / 2+\sqrt{B(t)}}
$$

This simple equation can be maximized depending on the different cuts $t$ to find the best cut value. $a$ is a constant and has to be chosen depending on the number of standard deviations corresponding to the confidence level at which the signal hypothesis is tested. 


\section{Chapter 7}

\section{Data and Event Selection}

\subsection{Data Sample and Selection}

The analyses that are described in the following sections were performed on data taken by the D $\varnothing$ detector between August 2002 and August 2008, corresponding to an integrated luminosity of $4.1 \mathrm{fb}^{-1}$ (see Fig. 3.3). Data taken with the detector before the Layer 0 installation, which happend in spring 2006, is named as Run IIa $\left(1.4 \mathrm{fb}^{-1}\right)$ and data after spring 2006 is named as Run $\mathrm{IIb}\left(2.7 \mathrm{fb}^{-1}\right)$. Two reduce the data a preselected subsample is used that contains only events with at least two reconstructed muons.

In order to get only good quality data, data quality monitoring is performed online and offline. The selection of good runs is based on information stored in the DØ Run Quality Database [87]. The requirements ensure that no hardware failures occurred and that all readout crates are included in the readout for the muon system [88], the SMT and the CFT. The tracking quality is particularly important for the identification of muons originating from the secondary vertex and for building the event variables.

\section{2 $B$-physics trigger strategy}

The most appealing features of hadron machines as a tool to study $B$-physics is their very high cross section for $b \bar{b}$ production and the access to all sorts of $b$-flavoured hadrons. On the other hand the fraction of $b$ events is small, about $0.1 \%-0.5 \%$ of the overall event rate and it is challenging to develop a trigger system to select the $b$ events. The goal of the $B$ physics trigger strategy [89] is to maximize the number of $B$-physics events being written to tape. The selection of the $B$ events often relies on semi-leptonic or multi-leptonic $B$ decays. A first background suppression can be achieved by triggering on a certain lepton $p_{t}$ threshold, as the leptons of $B$ decays tend to have a harder $p_{t}$ spectra than from 
minimum bias events. At low momenta the preferred choice of the leptons triggers are muons, since they are easily identified at trigger level. At Tevatron dimuon triggers are used for lifetime and mass measurements while the single muon events are exploited for mixing analysis. In addition there are triggers on displaced vertices.

\subsubsection{Muon Triggers}

All the decays studied in this thesis contain two muons in the final state. Triggering events starts in the L1 muon trigger system. The muon signatures at L1 are separated into four categories based on lose and tight requirements in either muon scintillator or wire chambers [90]. At L2 these muon candidates are then matched to tracks in the central tracker, and the trigger is fired if the tracks of the matched muon candidates satisfy a pre-defined $p_{t}$ cut and a track match quality cut. All dimuon triggers are based on muon scintillator hits at L1, a muon quality requirement at L2 and different criteria at L3. The main dimuon triggers are based on the muon system only, therefore taking the advantage of the full $\eta$ coverage of the muon system, unlike track based triggers. Currently, D $\varnothing$ has an approximate bandwidth limit at L3 of writing $50 \mathrm{~Hz}$ to tape where $10 \%$ of this rate is allocated to $B$ physics. The dimuon trigger has a present $\mathrm{L} 3$ rate of about $2 \mathrm{~Hz}$ [20].

\section{Dimuon Triggers}

The L1 and L2 requirements are the same for most of the used dimuon triggers. The L1 condition, abbreviated mu2ptxatxx, requires two muons (mu2), without a specific $p_{t}$ threshold (ptx), anywhere in the detector (a), with tight scintillator hits (t), but with no wire hits $(\mathbf{x x})$. The L2 condition requires at least one medium muon. The L3 conditions often require some $p_{t}$ threshold for the muons or an additional track. As we try to maximize the number of events all dimuon triggers have been used except those triggers which require some missing energy. The main dimuon trigger used for $B$ physics is DMU1_1L1MM2 which uses the same requirements as mu2ptxatxx for L1 and requires at least a medium muon at L2. In Table 7.1 an overview of some dimuon triggers is given.

\subsection{Monte Carlo Simulation (MC)}

Monte Carlo samples with about 200k events of the following decays, which are under study in this thesis were generated:

- $B^{ \pm} \rightarrow K^{ \pm} \mu^{+} \mu^{-}$ 


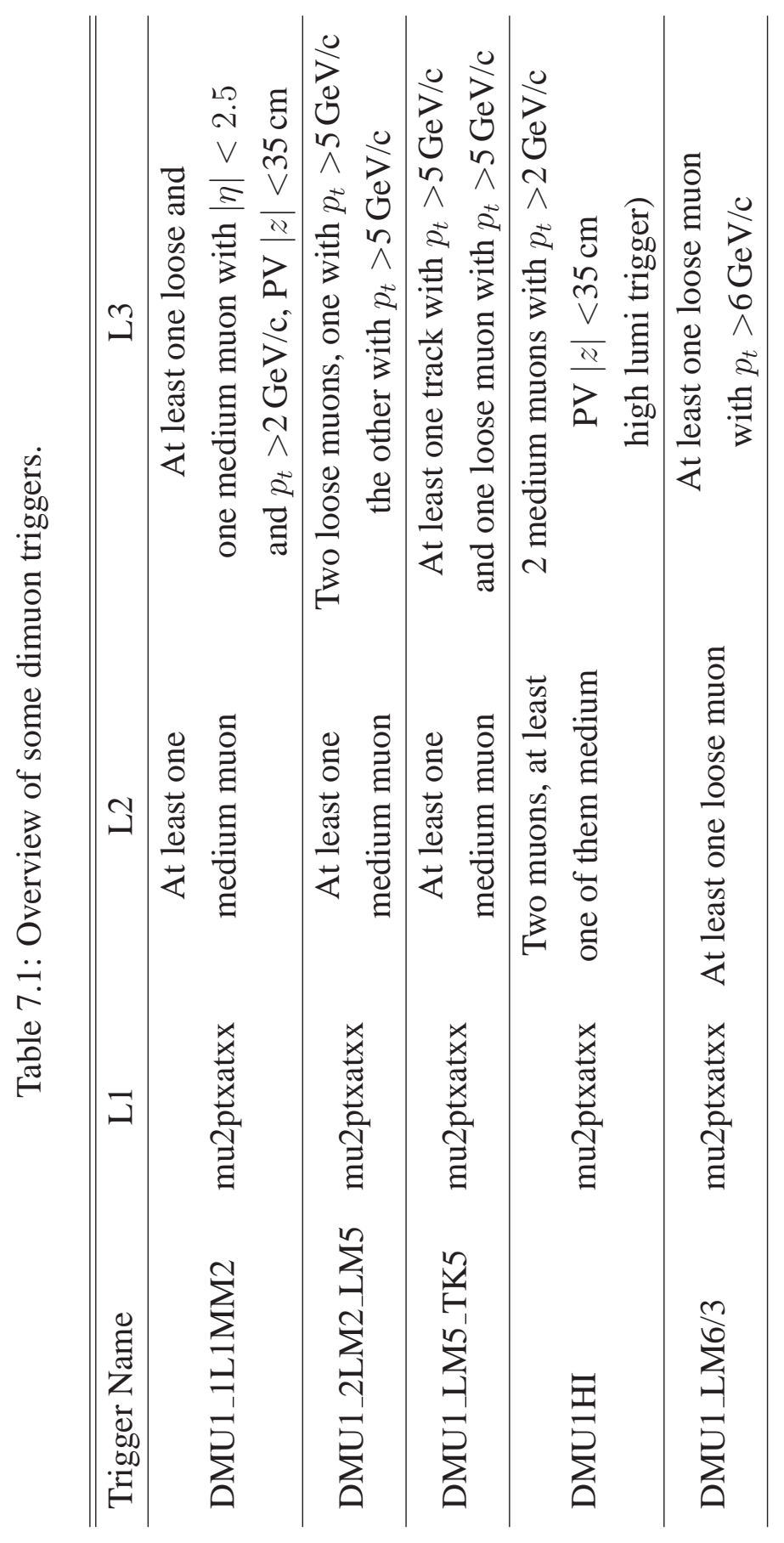


- $B^{ \pm} \rightarrow J / \psi K^{ \pm}$

- $B_{d}^{0} \rightarrow K^{*} \mu^{+} \mu^{-}$

- $B_{d}^{0} \rightarrow J / \psi K^{*}(892)$

As the detector layout changed from Run IIa to Run IIb Monte Carlo samples for both Runs had to be generated. The Monte Carlo version for Run IIa is known as p17 and for Run IIb it is called p20. For all samples, PYTHIA [91] was used for the generation of events. For the $B$-decay samples, a modified version of the program EvtGen [92] was used to simulate the $B$-decays. EvtGen has been developed for the simulation of $B$-decays at colliders operating at the $\Upsilon(4 \mathrm{~S})$ resonance, such as the PEP-II collider at SLAC [93] and the KEKB collider at KEK [94]. At these colliders the two $B$ mesons are predominantly produced by the decay of $\Upsilon(4 \mathrm{~S})$, and the two $B$-mesons are in a coherent quantum state. This coherence does not exist between $B$-meson pairs produced at a hadron collider. The version of the EvtGen program used at $\mathrm{D} \emptyset$ has therefore been modified to remove the coherence between the $B$-mesons.

PYTHIA and EvtGen were used to generate $b \bar{b}$ events with the condition for the $b$ quarks $p_{t}^{b}>0.1 \mathrm{GeV} / \mathrm{c}$ and $\left|\eta^{b}\right|<4.2$ required on the parton level.

After the event generation, the DØ Mess package (DØ Monte Carlo Event Selection System) [95] is used to apply cuts on generator level, that go beyond the selections that are offered by the generator or EvtGen. On all Monte Carlo samples, the following requirements were applied at generator level:

- $p_{t}$ of the $B$ candidate $>4.0 \mathrm{GeV} / \mathrm{c}$

- At least two opposite charged muons with

- muon $p_{t}>1.5 \mathrm{GeV} / \mathrm{c}$

- $|\eta|<2.1$ for each muon

- $p_{t}$ of the kaon and pion had to be larger than $0.5 \mathrm{GeV} / \mathrm{c}$

- $p_{t}$ of the $\mathrm{J} / \psi$ had to be larger than $4 \mathrm{GeV} / \mathrm{c}$

The two muons were required to come from the $B$-meson in the decays $B^{ \pm} \rightarrow K^{ \pm} \mu^{+} \mu^{-}$, $B_{d}^{0} \rightarrow K^{*} \mu^{+} \mu^{-}$and from the $J / \psi$ in the other cases. For all decays these cuts have different efficiencies which are $7.2 \%$ for $B^{ \pm} \rightarrow J / \psi K^{ \pm}, 6 \%$ for $B^{ \pm} \rightarrow K^{ \pm} \mu^{+} \mu^{-}$, $4.0 \%$ for $B_{d}^{0} \rightarrow J / \psi K^{*}(892)$ and $3.7 \%$ for $B_{d}^{0} \rightarrow K^{*} \mu^{+} \mu^{-}$.

The DØ GSTAR program (DØ GEANT Simulation of the Total Apparatus Response) [96] is used to track the particles through the detector and simulate their interactions with the material of the detector. The DØ Sim [97] program simulates the detector 
response and signal digitization, based on the DØ GSTAR output. Finally, event reconstruction is performed by the DØ RECO [68] program. In all samples "minimum bias events" were overlaid. The number of "minimum bias events" was randomly distributed following a Poisson distribution with an average of 0.8 .

\subsection{Discriminating Variables}

The selected variables used in the analyses to distinguish between signal and background events are described in the following section. Fig. 7.1 shows a sketch of the discriminating variables for the decay $B^{ \pm} \rightarrow K^{ \pm} \mu^{+} \mu^{-}$.

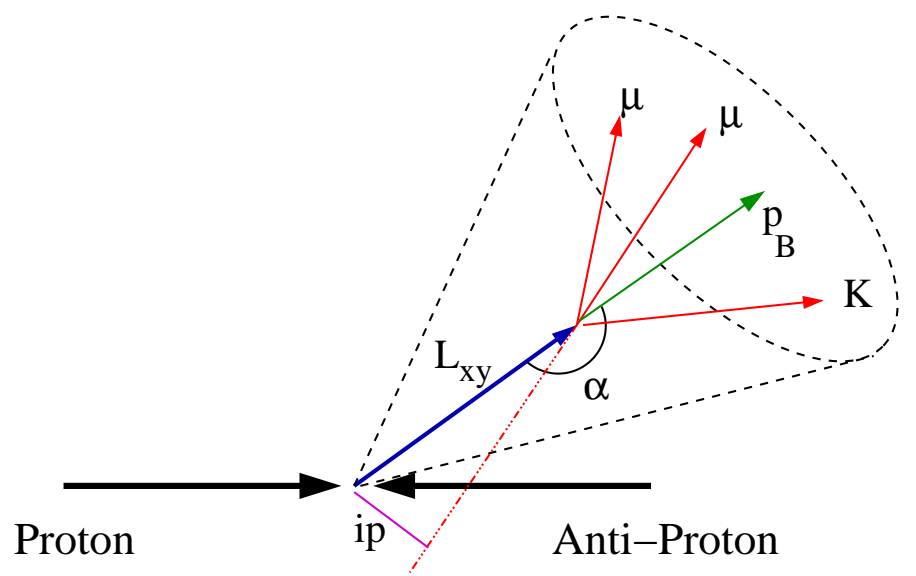

Figure 7.1: Pictogram for visualisation of the discriminating variables of the decay $B^{ \pm} \rightarrow$ $K^{ \pm} \mu^{+} \mu^{-}$.

\section{Transverse Decay Length Significance}

The $B^{ \pm}$and $B^{0}$ mesons have a lifetime of about $1.6 \mathrm{ps}$ [98] leading to a proper decay length of about $500 \mu \mathrm{m}$. This characteristic is a good quantity to reject background events. The transverse decay length $L_{x y}$ is calculated as

$$
L_{x y}=\frac{\vec{l}_{v t x} \cdot \vec{p}_{t}^{B}}{p_{t}^{B}}
$$

where $\vec{p}_{t}^{B}$ is the transverse momentum vector of the $B$ candidate and $\vec{l}_{v t x}$ is the vector pointing from the primary vertex to the secondary vertex. The discriminating variables used in the multivariate analysis is the transverse decay length significance $L_{x y} / \delta L_{x y}$ with $\delta L_{x y}$ described as the error on the transverse decay length. 


\section{Isolation}

Due to fragmentation characteristics it is expected that the $B$ meson carries most of the energy of the $b$ quark and thus the number of additional tracks close to the $B$ meson tends to be small. The isolation variable $\mathcal{I}$ is used as a second discriminating variable for the Boosted decision tree:

$$
\mathcal{I}=\frac{\left|\vec{p}\left(B^{ \pm}\right)\right|}{\left|\vec{p}\left(B^{ \pm}\right)\right|+\sum_{\text {track } i \neq B} p_{i}(\Delta \mathcal{R}<1)}
$$

Here, $\sum_{\text {track } i \neq B} p_{i}$ is the scalar sum over the momenta of all tracks excluding the muons and the kaon within a cone of $\Delta \mathcal{R}<1$ around the momentum vector $\vec{p}\left(B^{ \pm}\right)$of the $B$ meson, where $\Delta \mathcal{R}=\sqrt{(\Delta \phi)^{2}+(\Delta \eta)^{2}}$.

\section{Opening Angle $\alpha$}

The opening (or pointing) angle $\alpha$ is defined as the angle between the momentum vector $\vec{p}(B)$ of the $B$ candidate and the vector $\vec{l}_{v t x}$ between the primary and the secondary vertex. This angle should be around $0^{\circ}$ if the $B$ meson is built out of the right daughter particles. This variable ensures the consistency between the direction of the decay vertex and the momentum vector of the $B$ candidate.

\section{$\chi^{2}$ of secondary vertex}

The $\chi^{2}$ of the secondary vertex should be of the order $\chi^{2}=2 n-3$ where $n$ is the number of tracks.

\section{Impact Parameter}

To define the impact parameters, first the point of closest approach (PCA) is defined as projection of the track trajectory in the axial plane to the primary vertex. The axial impact parameter $\left(i p^{a}\right)$ is defined as the distance between the PCA and the vertex in the axial plane. The stereo impact parameter $\left(i p^{s}\right)$ is the difference of $\mathrm{z}$-coordinates between the PCA on the three dimensional track and the primary vertex.

- Minimal Axial Muon Impact Parameter The impact parameters of the two muons $\left(i p_{\mu}\right)$, originating from the displaced secondary vertex of a $B$ meson, tend to be large. In this analysis the projection of the impact parameter to the axial plane of 
the primary vertex is used. The multivariate analysis uses the minimal axial impact parameter significance of the two muons, defined as:

$$
i p_{\mu}=\operatorname{Min}\left(\left(\frac{i p_{\mu_{1}}^{a}}{\delta i p_{\mu_{1}}^{a}}\right)^{2} ;\left(\frac{i p_{\mu_{2}}^{a}}{\delta i p_{\mu_{2}}^{a}}\right)^{2}\right)
$$

where $\delta i p_{\mu}^{a}$ is the error on the axial impact parameter.

- Impact parameter significance of the kaon In this analysis the impact parameter significance is defined as $\left(i p_{P}^{a} / \delta i p_{P}^{a}\right)^{2}+\left(i p_{P}^{s} / \delta i p_{P}^{s}\right)^{2}$. The third $B$ meson decay particle, for example the charged kaon $K^{ \pm}$of $B^{ \pm} \rightarrow K^{ \pm} \mu^{+} \mu^{-}$also tends to have a large impact parameter with respect to the primary vertex. 


\section{Chapter 8}

\section{Rare Decay $B^{ \pm} \rightarrow K^{ \pm} \mu^{+} \mu^{-}$}

In this Chapter we present the search for the rare decay $B^{ \pm} \rightarrow K^{ \pm} \mu^{+} \mu^{-}$and a measurement of its branching fraction. For normalisation purposes the decay $B^{ \pm} \rightarrow J / \psi K^{ \pm}$is being used as described in section 2.6. The decay $B^{ \pm} \rightarrow K^{ \pm} \mu^{+} \mu^{-}$only occurs via box and penguin diagrams as shown in Fig. 8.1. At D $\varnothing$ the inclusive search for $b \rightarrow s \mu^{+} \mu^{-}$ was performed earlier in Run I and a limit of $b \rightarrow s \mu^{+} \mu^{-}<3.2 \cdot 10^{-4}$ at $90 \% \mathrm{CL}$ was set using $\mathcal{L}=50 \pm 2.7 \mathrm{pb}^{-1}$ [99].
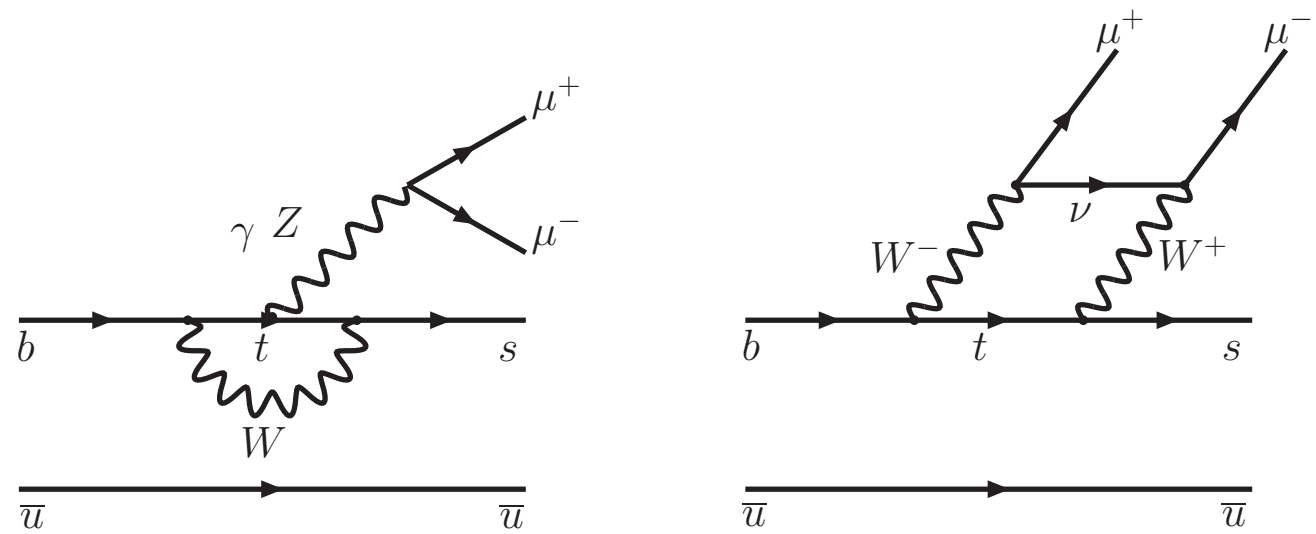

Figure 8.1: SM diagrams for the decay $B^{ \pm} \rightarrow K^{ \pm} \mu^{+} \mu^{-}$.

\subsection{Introduction}

For the decay $B^{ \pm} \rightarrow K^{ \pm} \mu^{+} \mu^{-}$we have three tracks in the final state. The two muons should give a clear signature and then a third track is added. Looking at the dimuon spectra of data and Monte Carlo in Fig.8.2 several resonances can be seen in the data. 
The branching fractions of the decays with a $J / \psi$ or a $\psi$ in the final state are orders of magnitude larger than that of the non-resonant decay $B^{ \pm} \rightarrow K^{ \pm} \mu^{+} \mu^{-}$.
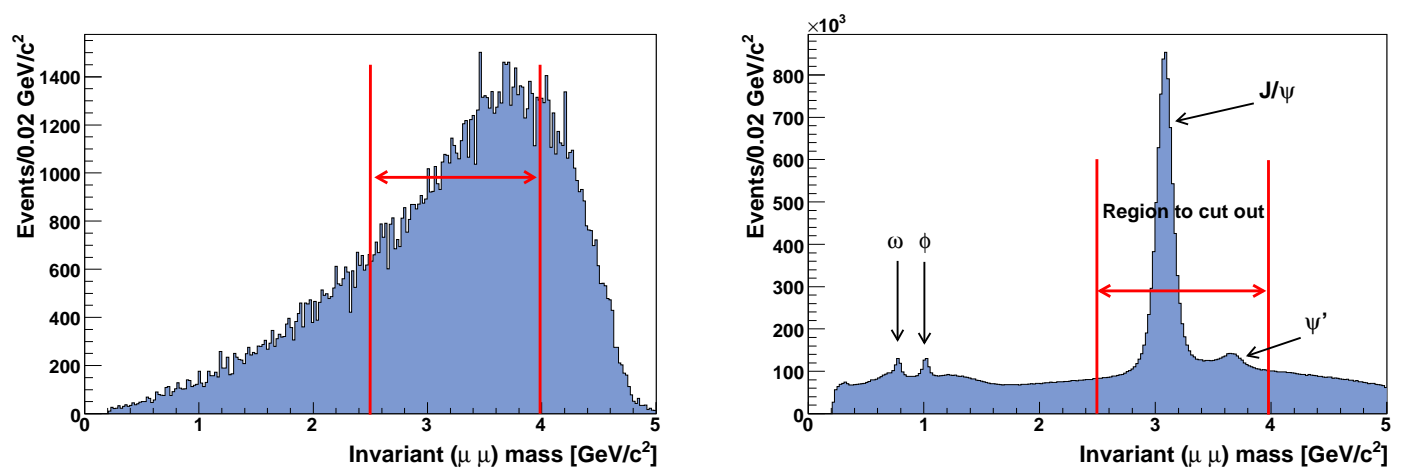

Figure 8.2: The dimuon invariant mass distribution of the Monte Carlo (left) and data (right).The region between the red lines is removed in the dimuon invariant mass spectra of the decays $B^{ \pm} \rightarrow K^{ \pm} \mu^{+} \mu^{-}$and $B_{d}^{0} \rightarrow K^{*} \mu^{+} \mu^{-}$.

The $J / \psi$ mass width in data and in Monte Carlo is fitted by a single Gaussian (see Fig. 8.3 ), resulting in a mass resolution of $\sigma=65 \mathrm{MeV} / \mathrm{c}^{2}$ for Monte Carlo and $\sigma=75 \mathrm{MeV} / \mathrm{c}^{2}$ for data, respectively.
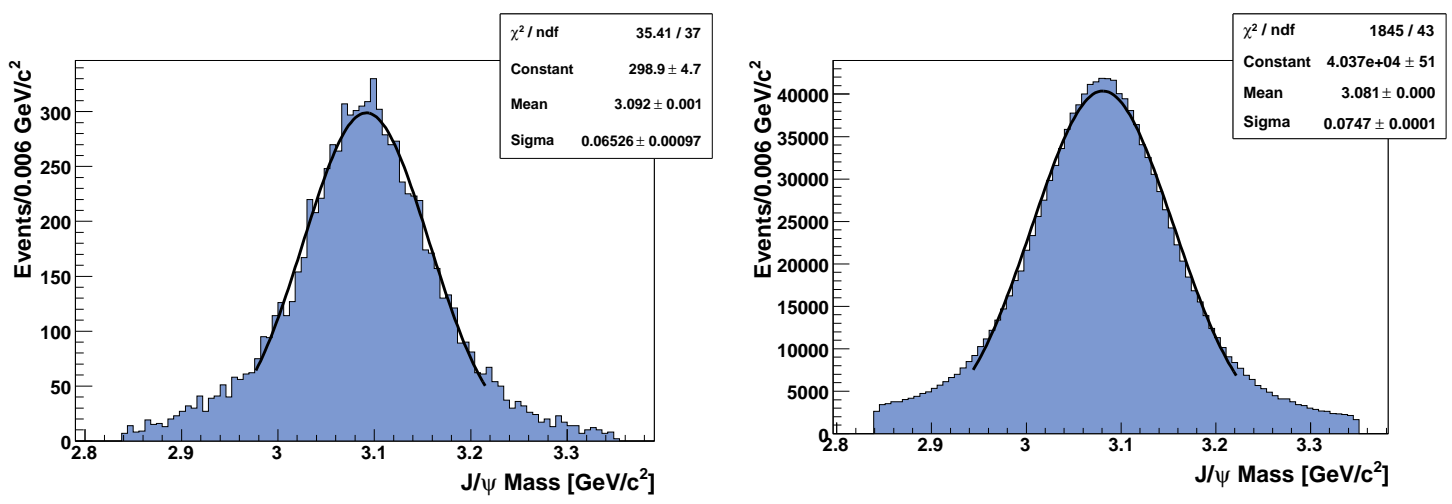

Figure 8.3: The $\mathrm{J} / \psi$ resolution in Monte Carlo $\left(\sigma=65 \mathrm{MeV} / \mathrm{c}^{2}\right)$ on the left and in Data $\left(\sigma=75 \mathrm{MeV} / \mathrm{c}^{2}\right)$ on the right side.

For the calibration process $B^{ \pm} \rightarrow J / \psi K^{ \pm}$the invariant $\mu^{+} \mu^{-}$mass is required to be in the range of $2.84 \mathrm{GeV} / \mathrm{c}^{2}<m_{\mu^{+} \mu^{-}}<3.35 \mathrm{GeV} / \mathrm{c}^{2}$. For the signal process $B^{ \pm} \rightarrow$ $K^{ \pm} \mu^{+} \mu^{-}$the mass regions of the resonances $J / \psi \rightarrow \mu^{+} \mu^{-}$and $\psi(2 S) \rightarrow \mu^{+} \mu^{-}$ $\left(2.5 \mathrm{GeV} / \mathrm{c}^{2}<m_{\mu^{+} \mu^{-}}<4.04 \mathrm{GeV} / \mathrm{c}^{2}\right)$ are excluded. This mass exclusion region corresponds to $\pm 8 \sigma$ of the $J / \psi$ mass in order to make sure that no noticeable contribution from the $J / \psi$ tail leaks into the signal window. For the $\psi(2 S)$ resonance the same resolution as for the $J / \psi$ was taken but only $\pm 5 \sigma$ is excluded. 


\subsection{Pre-selection}

A medium quality for the two muons is required. Both muons need to satisfy $p_{t} \geq 2.5 \mathrm{GeV} / \mathrm{c}$ and have to be in the central rapidity region $|\eta| \leq 2.0$. They need to have at least 2 hits in the Silicon Microstrip Tracker (SMT) and 2 hits in the Central Fiber Tracker (CFT). The sum of their charges has to be zero. The third particle is required to have $p_{t}>0.5 \mathrm{GeV} / \mathrm{c}$ and at least one hit in the SMT. A secondary vertex is built out of these three particles and a $\chi^{2}<35$ of the vertex fit is required.

After the secondary vertex is found, the invariant mass of the three particles is calculated. As there are more than one $B$ candidate in each event, the candidate with the highest $p_{t}$ of the $K$ in each event is selected. All preselection cuts are summarized in Table 8.1.

Table 8.1: Preselection cuts for the $B^{ \pm} \rightarrow K^{ \pm} \mu^{+} \mu^{-}$candidates. For the calibration channel $B^{ \pm} \rightarrow J / \psi K^{ \pm}$the same cuts are applied except for the invariant $\mu \mu$ mass where the range $2.84 \mathrm{GeV} / \mathrm{c}^{2}<m_{\mu^{+} \mu^{-}}<3.35 \mathrm{GeV} / \mathrm{c}^{2}$ is selected.

\begin{tabular}{l|c}
\hline \hline Cuts & value \\
\hline \hline muon quality & medium \\
\hline sum of muon charge & 0 \\
\hline muon $p_{t}^{\mu}$ & $p_{t}^{\mu} \geq 2.5 \mathrm{GeV} / \mathrm{c}$ \\
\hline pseudo rapidity of muons & $\left|\eta_{\mu}\right| \leq 2.0$ \\
\hline muon tracking hits & $2 \mathrm{SMT}, 2 \mathrm{CFT}$ \\
\hline third particle & $p_{t}>0.5 \mathrm{GeV} / \mathrm{c} ; 1 \mathrm{SMT}$ hit \\
\hline excluded mass window & $2.50 \mathrm{GeV} / \mathrm{c}^{2}<\mathrm{M} \mu \mu$ \\
\hline Vertex $\chi^{2}$ & $\chi^{2}<35$ \\
\hline best candidate & highest $p_{t}^{K}$ \\
\hline \hline
\end{tabular}

For the further analysis the mass resolution of the $B^{ \pm} \rightarrow K^{ \pm} \mu^{+} \mu^{-}$decay has to be determined. The $\mu \mu K^{ \pm}$mass distributions of the Monte Carlo $B^{ \pm} \rightarrow K^{ \pm} \mu^{+} \mu^{-}$decay and the $J / \psi K^{ \pm}$mass distribution of the calibration channel $B^{ \pm} \rightarrow J / \psi K^{ \pm}$with no mass constraint on the $J / \psi$ mass are compared in Fig. 8.4. The resolutions in the two distributions, as determined by a single Gaussian fit, are in good agreement. The $J / \psi$ 
mass fits in Fig. 8.3 demonstrate that the mass resolution obtained in Monte Carlo is a few percent better than in data and thus we scale the observed $B$ mass resolution in Monte Carlo $B^{ \pm} \rightarrow K^{ \pm} \mu^{+} \mu^{-}$events in order to obtain a more precise expectation of the $B$ mass resolution in data. To determine the resolution in data for $B^{ \pm} \rightarrow K^{ \pm} \mu^{+} \mu^{-}$the following Equation is used:

$$
\left(\Delta M_{\text {data }}\right)^{2}=\left(\Delta M_{\text {MonteCarlo }}\right)^{2}+\left(\Delta M_{\text {unknown }}\right)^{2}
$$

There are some unknown effects which enlarge the resolution in data compared to Monte Carlo. This is taken into account in the term $\Delta M_{\text {unknown }}$ in Equation 8.1. $\Delta M_{\text {unknown }}$ is determined by the $J / \psi$ mass resolution of the normalisation channel $B^{ \pm} \rightarrow J / \psi K^{ \pm}$(see Fig. 8.3) and so a resolution of $100 \mathrm{MeV} / c^{2}$ is calculated for the $B^{ \pm} \rightarrow K^{ \pm} \mu^{+} \mu^{-}$channel in data.
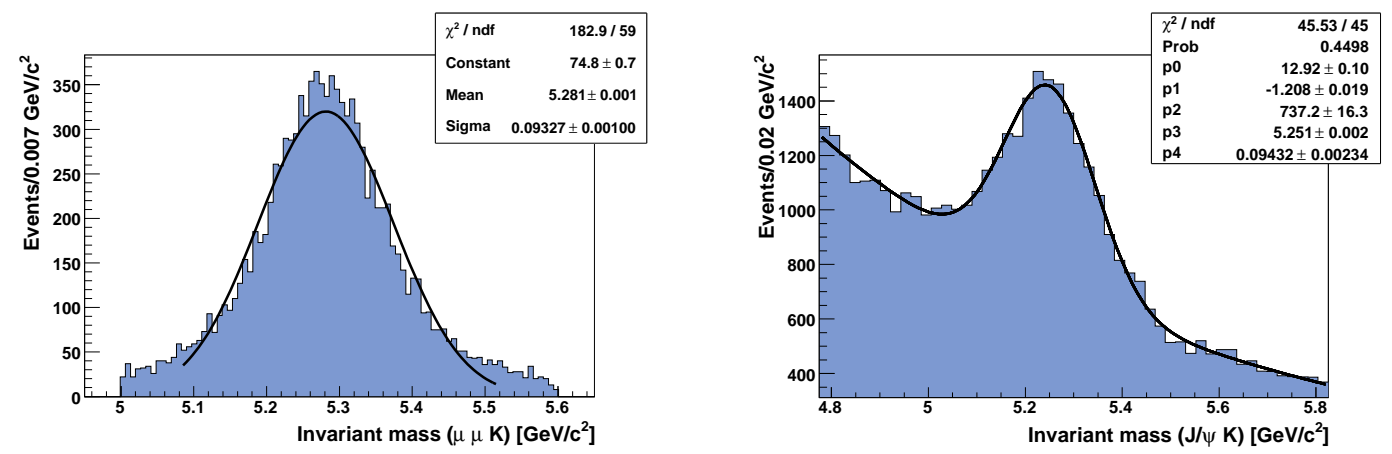

Figure 8.4: The $B^{ \pm} \rightarrow K^{ \pm} \mu^{+} \mu^{-}$invariant mass distributions of the signal Monte Carlo (left histogram) and of the calibration data $\left(B^{ \pm} \rightarrow J / \psi K^{ \pm}\right.$) (no mass constraint on the $\mathrm{J} / \psi)$. The Gaussian fit results in mass widths of $\sigma=(93 \pm 1) \mathrm{MeV} / \mathrm{c}^{2}$ for the Monte Carlo and of $\sigma=(94 \pm 2) \mathrm{MeV} / \mathrm{c}^{2}$ for the data.

\subsection{Trigger Simulation}

The trigger is simulated by reweighting the Monte Carlo events according to a threshold function applied to the $p_{t}$ of the muons. In addition it turns out, that the simulated $p_{t}^{B}$ distribution of the calibration channel does not completely match the data, therefore an additional weighting function for the $p_{t}^{B}$ distribution has been determined as in other $B$ physics analyses [100]. The trigger threshold functions and the $p_{t}^{B}$ weighting are correlated, thus an iterative procedure to optimize the weighting functions was chosen.All weighting functions are always applied eventwise. The trigger simulation and $p_{t}^{B}$ weighting is performed for p17 Monte Carlo which is used for Run IIa and p20 Monte Carlo used for Run IIb independently. In this section we show the results for Run IIa data and p17 Monte Carlo. 
The method to determine the threshold function for a single muon trigger is described in Ref. [101]. A function of the type

$$
f\left(p_{t}\right)=\frac{2 a-d}{2} \cdot \operatorname{erf}\left[b\left(p_{t}-c\right)\right]+\frac{d}{2}
$$

was used with

$$
\operatorname{erf}=\frac{2}{\sqrt{\pi}} \int_{0}^{x} e^{-t^{2}} d t
$$

In our case two muons are required in the trigger. Two different threshold values need to be applied to the first (higher $p_{t}$ ) and second (lower $p_{t}$ ) muon, respectively. In order to determine the trigger function, the data of the $J / \psi K$ calibration dataset after the preselection as described in section 8.2 is used, without the $p_{t}^{\mu}$ cut and only requiring a loose muon quality. In order to obtain a higher-purity sample of $B^{ \pm} \rightarrow J / \psi K^{ \pm}$events for the calculation of the reweighting function an additional cut on the decay length significance is applied requiring the $B$ candidate to be separated from the primary vertex by at least 4.0 standard deviations. The effect of this additional criterion is demonstrated in Fig. 8.5. This cut is not part of the main event selection.
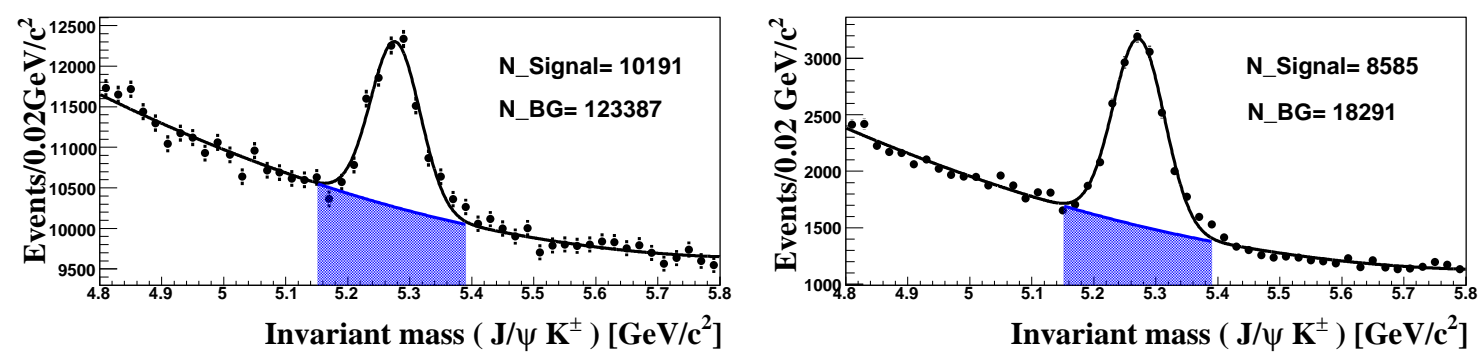

Figure 8.5: The calibration data $B^{ \pm} \rightarrow J / \psi K^{ \pm}$after the preselection cuts (left) and with the additional decay length significance cut (right). This additional cut is only used for the trigger calibration of the Monte Carlo. Note that the zero on the vertical scale is suppressed.

For the first muon $\left(p_{t}^{\mu 1}\right)$, the parameters of the threshold function were taken from the single muon trigger, described in [101]. For the second muon $\left(p_{t}^{\mu 2}\right)$, all parameters of the threshold function were redetermined, using a similar method as in [101]. Two data sets were compared: The preselected data described above, and a sample that was obtained by applying all pre-selection cuts except the dimuon trigger. Fig. 8.6 (right) shows the ratio of the $p_{t}^{\mu 2}$ distributions of the two data sets. To this histogram the same threshold function as described in Equation 8.2 is fitted to obtain the parameters for the second muon. After applying these weights to the Monte Carlo $p_{t}^{\mu}$ spectra there are still some discrepancies between data and Monte Carlo (see Fig. 8.7). 

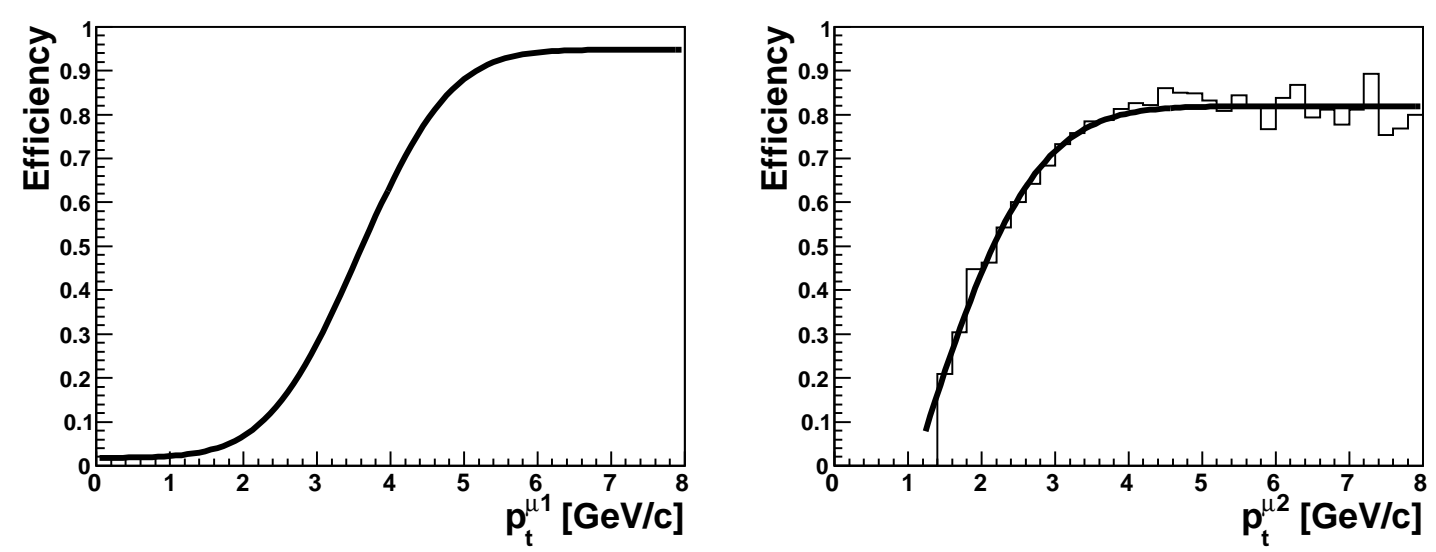

Figure 8.6: The trigger threshold function for $p_{t}^{\mu 1}$ (left) and $p_{t}^{\mu 2}$ (right) to be applied to the Monte Carlo events. The histogram in the right figure represents the ratio of the two data selections, with and without the dimuon trigger requirement. The bin size of the histo is $0.2 \mathrm{GeV} / \mathrm{c}$
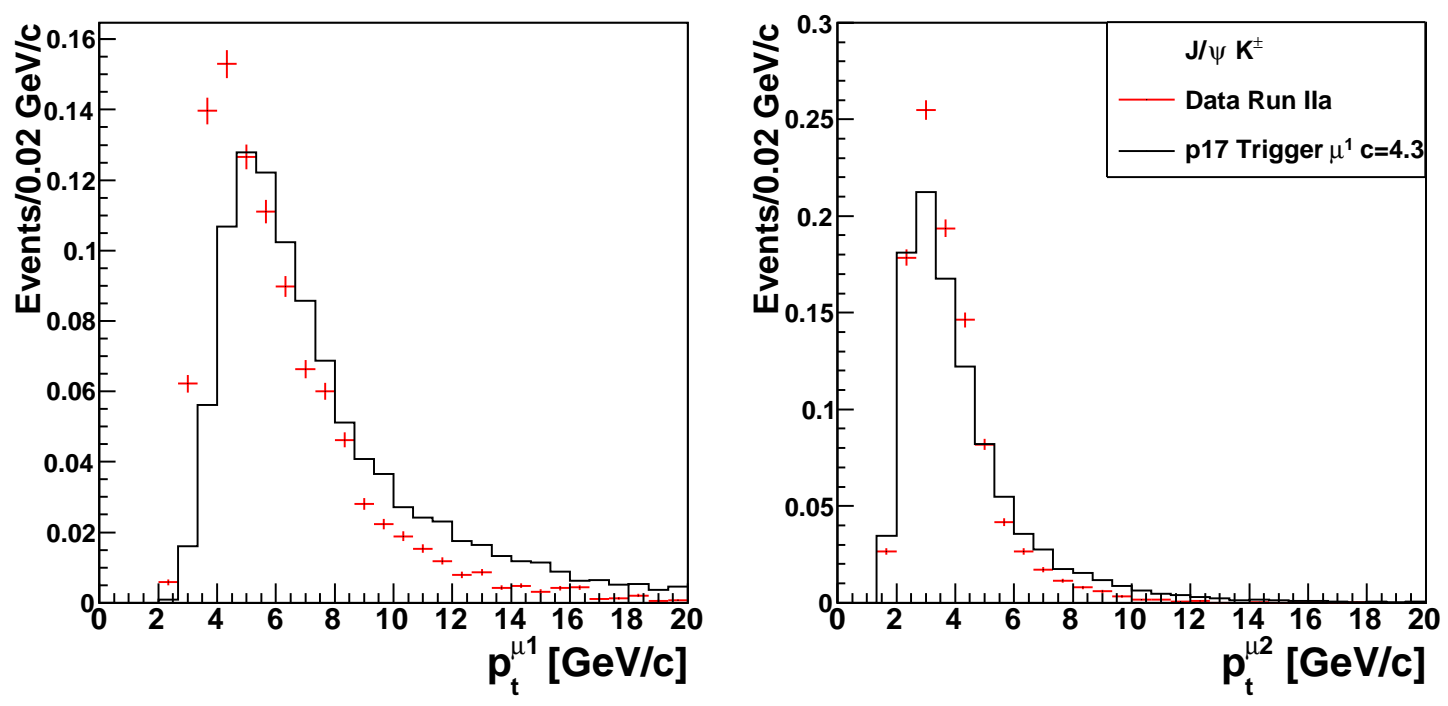

Figure 8.7: The $p_{t}^{\mu 1}$ (left) and $p_{t}^{\mu 2}$ (right) distributions for data after preselection (crosses) and Monte Carlo (line) events, after reweighting with the two trigger threshold functions.

Also the $p_{t}^{B}$ distribution shows some disagreement between data and Monte Carlo. In order to correct for this, an additional exponential reweighting function is determined using the calibration channel $B^{ \pm} \rightarrow J / \psi K^{ \pm}$. 
The ratio of the $p_{t}^{B}$ distributions of $B^{ \pm} \rightarrow J / \psi K^{ \pm}$in data and Monte Carlo is shown in Fig. 8.9. It is fitted with an exponential function:

$$
f\left(p_{t}^{B}\right)=\exp \left(s+t \cdot p_{t}^{B}\right)
$$

This weight is applied to the Monte Carlo distributions in addition to the trigger weights. The resulting $p_{t}^{\mu}$ distributions can be seen in Fig. 8.8. The threshold of data and Monte Carlo of the $p_{t}^{\mu 1}$ shows still some discrepancies. As the $p_{t}^{\mu 1}$ spectra for the dimuon trigger is expected to be softer than for the single muon trigger, the parameter $c$ of the trigger function 8.2 of the first muon, which describes the threshold, is refitted to the data.
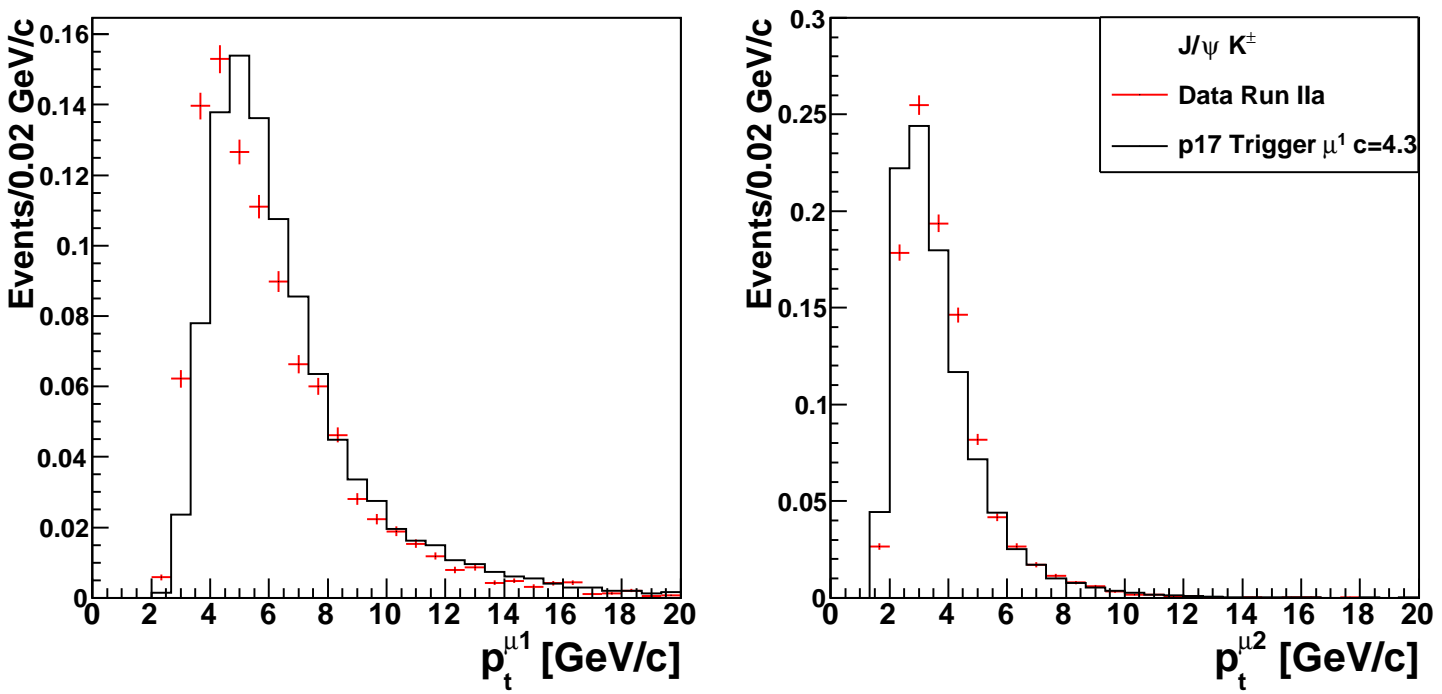

Figure 8.8: The $p_{t}^{\mu 1}$ (left) and $p_{t}^{\mu 2}$ (right) distributions for data after preselection (crosses) and Monte Carlo (line) events, after reweighting the Monte Carlo events with the two trigger threshold functions and applying the $p_{t}^{B}$ weight.

As a second iteration of the weighting procedure, the $p_{t}^{B}$ distributions are then compared again and the final weighting functions are obtained. The fit of the $p_{t}^{B}$ ratio with Equation 8.4 results in $s=0.784 \pm 0.0550$ and $t=-0.052 \pm 0.003$. Due to the discrepancies on low $p_{t}^{B}$ an additional cut on $p_{t}^{B} \geq 8 \mathrm{GeV} / \mathrm{c}$ is applied on both Monte Carlo and data. The $p_{t}^{B}$ spectra before and after applying the $p_{t}^{B}$ weights can be seen in Fig. 8.10. The plots in Fig. 8.11 show the $p_{t}$ spectra of the muons in Monte Carlo and data after applying the weight-functions. Due to this reweighting procedure a satisfactory agreement between data and Monte Carlo could be obtained. The resulting parameters of the threshold functions for both muons are given in Table 8.2. All the discriminating variables of the decay $B^{ \pm} \rightarrow J / \psi K^{ \pm}$are compared in Monte Carlo and data to see if the weighting is correct and can bee seen in Fig. 8.12. 


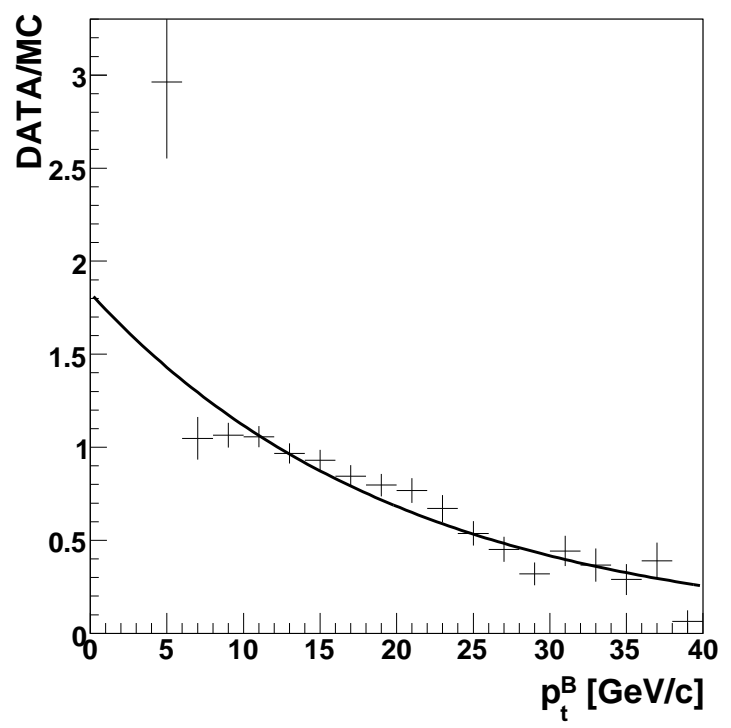

Figure 8.9: Weighting function determined from the ratio of $p_{t}^{B}$ distributions for Run IIa data and for p17 Monte Carlo, for the calibration channel $B^{ \pm} \rightarrow J / \psi K^{ \pm}$.
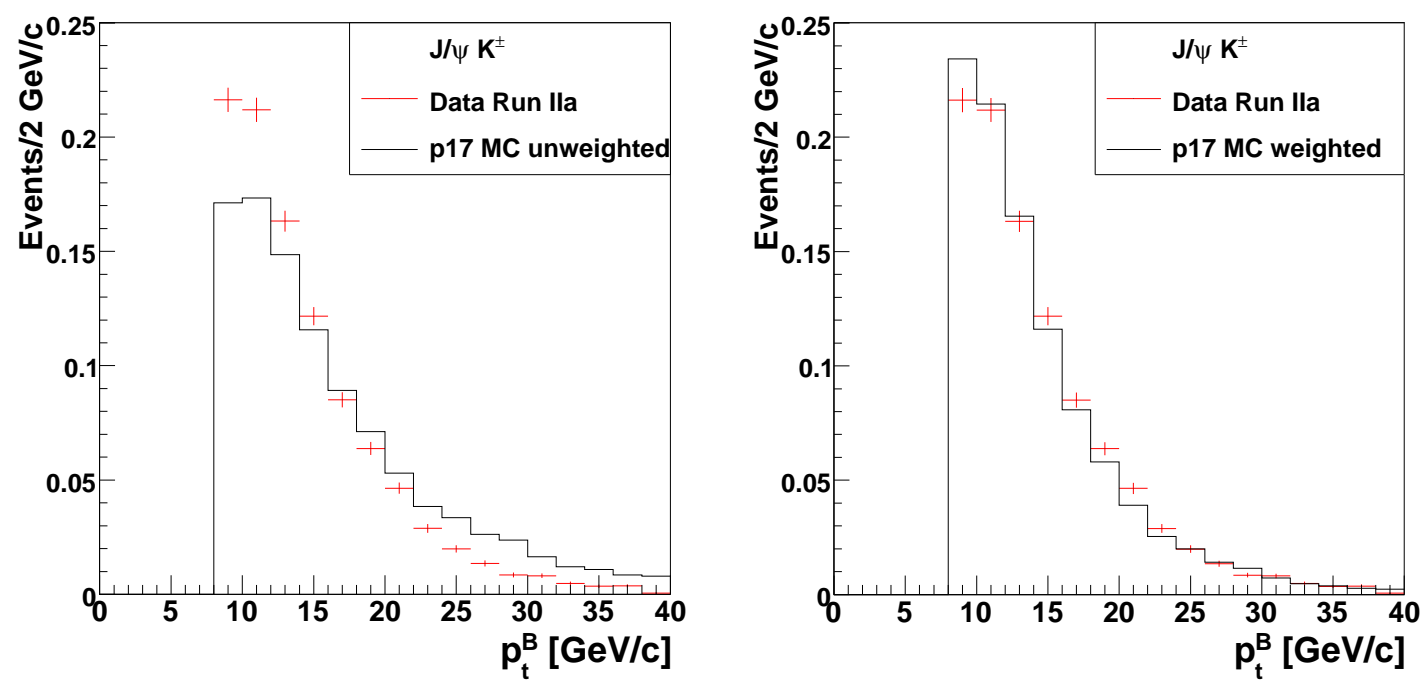

Figure 8.10: $p_{t}^{B}$ distributions of the $B^{ \pm} \rightarrow J / \psi K^{ \pm}$calibration channel. The Monte Carlo distribution (line) is compared to the preselected data (crosses) before (left) and after (right) applying the $p_{t}$ reweighting function. 

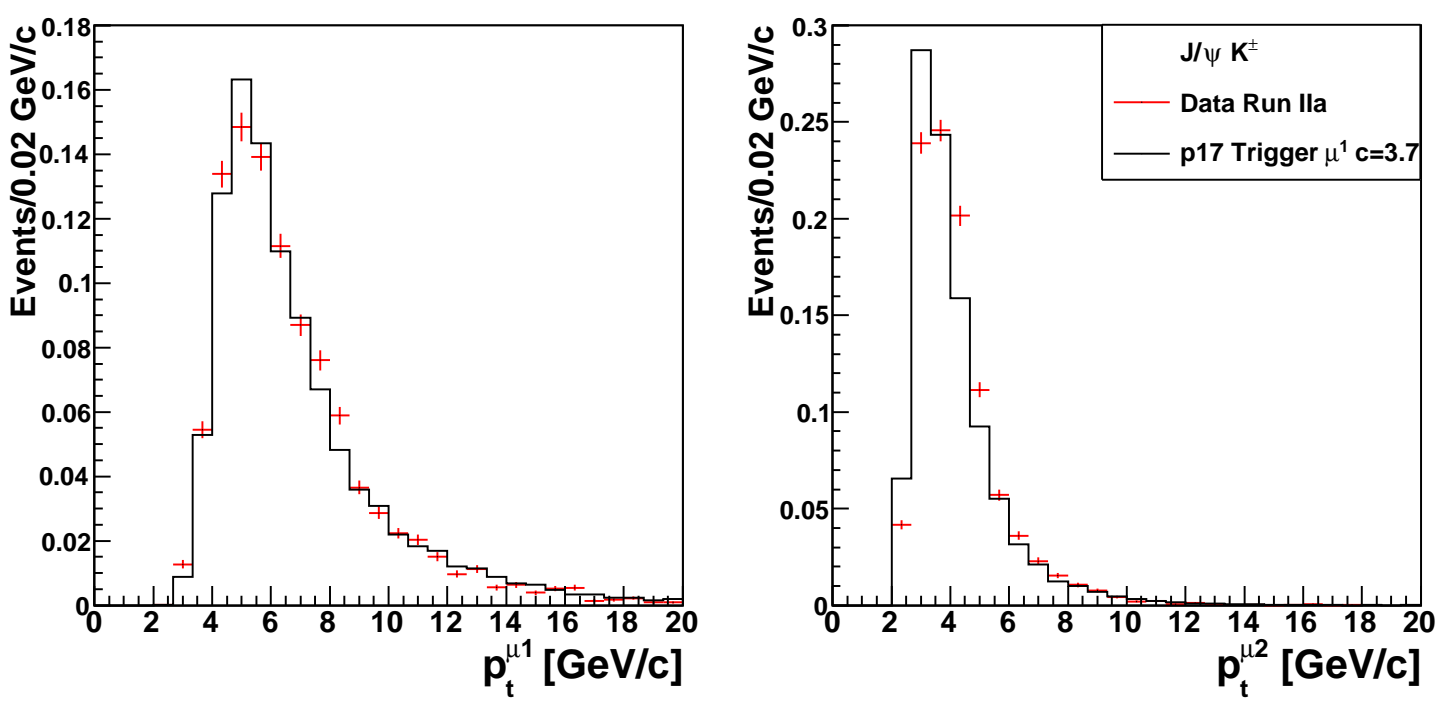

Figure 8.11: The $p_{t}^{\mu 1}$ (left) and $p_{t}^{\mu 2}$ (right) distributions for data after preselection (crosses) and Monte Carlo (line) events, after reweighting with the corrected trigger threshold functions and the $p_{t}^{B}$ weighting function (after final iteration).

Table 8.2: Parameters of the Monte Carlo trigger threshold function

\begin{tabular}{|c|c|c|c|c|}
\hline \hline & $\mathrm{a}$ & $\mathrm{b}$ & $\mathrm{c}$ & $\mathrm{d}$ \\
\hline \hline $\mathrm{p} 17 f\left(p_{t}^{\mu 1}\right)$ & 0.9477 & 0.7223 & 3.683 & 0.9656 \\
\hline $\mathrm{p} 17 f\left(p_{t}^{\mu 2}\right)$ & 0.7739 & 0.5974 & 1.061 & $\approx 0$ \\
\hline \hline $\mathrm{p} 20 f\left(p_{t}^{\mu 1}\right)$ & 0.9693 & 0.9593 & 3.702 & 0.98 \\
\hline $\mathrm{p} 20 f\left(p_{t}^{\mu 2}\right)$ & 0.8510 & 0.6411 & 1.04 & $\approx 0$ \\
\hline \hline
\end{tabular}

\subsection{Final selection - TMVA}

To obtain the best possible distinction between signal and background a six-variable multivariate analysis (MVA) is performed. The Toolkit for Multivariate Data Analysis (TMVA) implemented in the ROOT analysis software is used for this purpose.

Due to the exclusion of the of $J / \psi$ and $\psi(2 S)$ resonances in the invariant mass of the dimuon system, no peaking background is expected in the signal window. A background sample is obtained from the data after preselection in the upper $\left(4.379 \mathrm{GeV} / \mathrm{c}^{2}\right.$ to $\left.4.979 \mathrm{GeV} / \mathrm{c}^{2}\right)$ and lower $\left(5.579 \mathrm{GeV} / \mathrm{c}^{2}\right.$ to $\left.6.179 \mathrm{GeV} / \mathrm{c}^{2}\right)$ sidebands of the invari- 

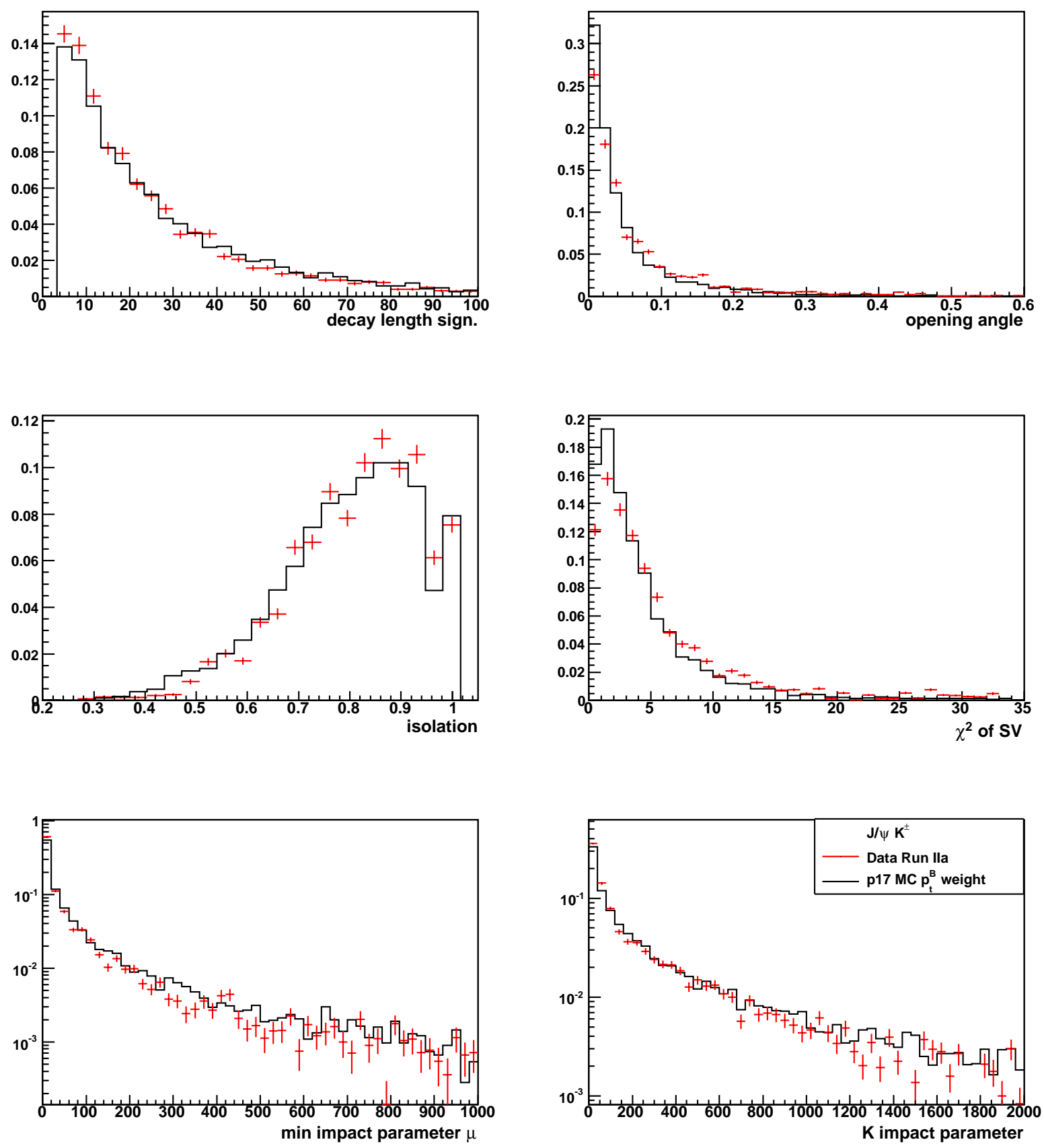

Figure 8.12: The discriminating variables of the calibration channel of Monte Carlo (line) and data (crosses) after applying all the weighting functions to the Monte Carlo. 


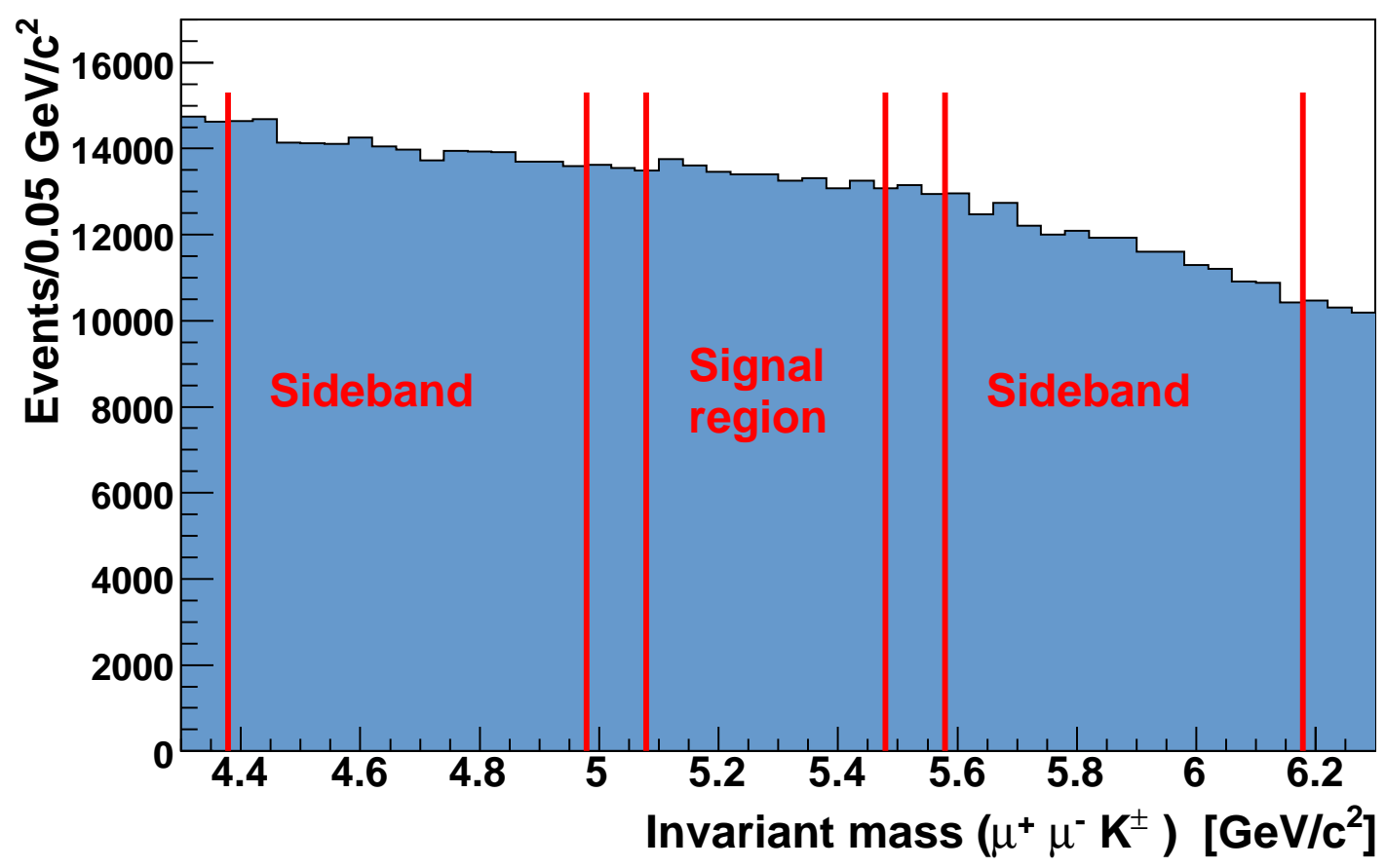

Figure 8.13: The definition of the sidebands $(-9 \sigma$ to $-3 \sigma),(+3 \sigma$ to $+9 \sigma)$ and signal region $(-2 \sigma$ to $+2 \sigma)$ for the data of the decay $B^{ \pm} \rightarrow K^{ \pm} \mu^{+} \mu^{-}$. The mass resolution in the data is assumed to be $\sigma=100 \mathrm{MeV} / \mathrm{c}^{2}$ as described in section 8.2

ant $\mu \mu K^{ \pm}$mass distribution (see Fig. 8.13). The signal sample consists of the preselected $B^{ \pm} \rightarrow K^{ \pm} \mu^{+} \mu^{-}$Monte Carlo events in the mass window of $\left(5.079 \mathrm{GeV} / \mathrm{c}^{2}\right.$ to $\left.5.479 \mathrm{GeV} / \mathrm{c}^{2}\right)$.

The following variables are used in the MVA:

- Transverse decay length significance (see Fig. 8.14)

- Isolation variable (see Fig. 8.14)

- Opening angle (see Fig. 8.15)

- Vertex quality (see Fig. 8.15)

- Muon impact parameter (see Fig. 8.16)

- Kaon impact parameter (see Fig. 8.16) 

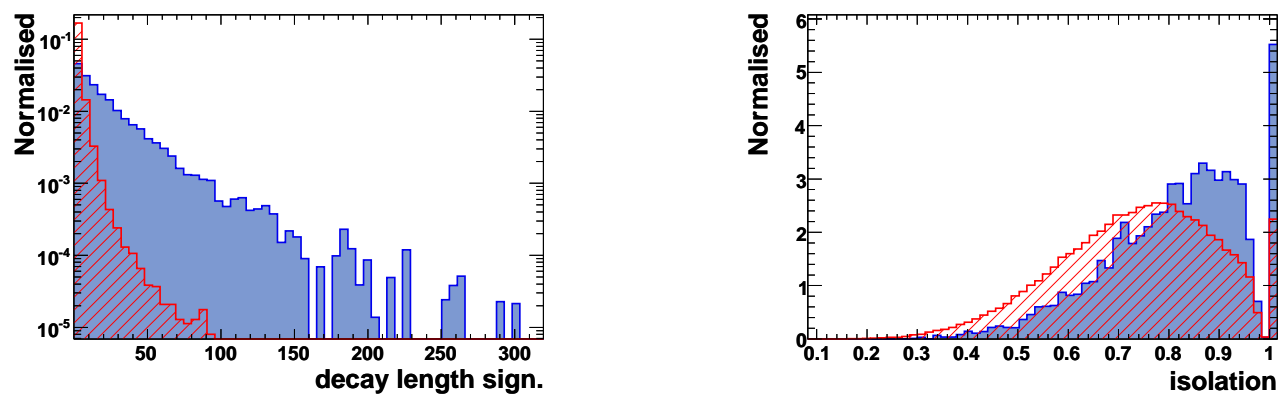

Figure 8.14: The transverse decay length significance is shown on the left side. On the right the isolation variable can be seen. The filled histogram is the signal and the shaded histogram is the background.
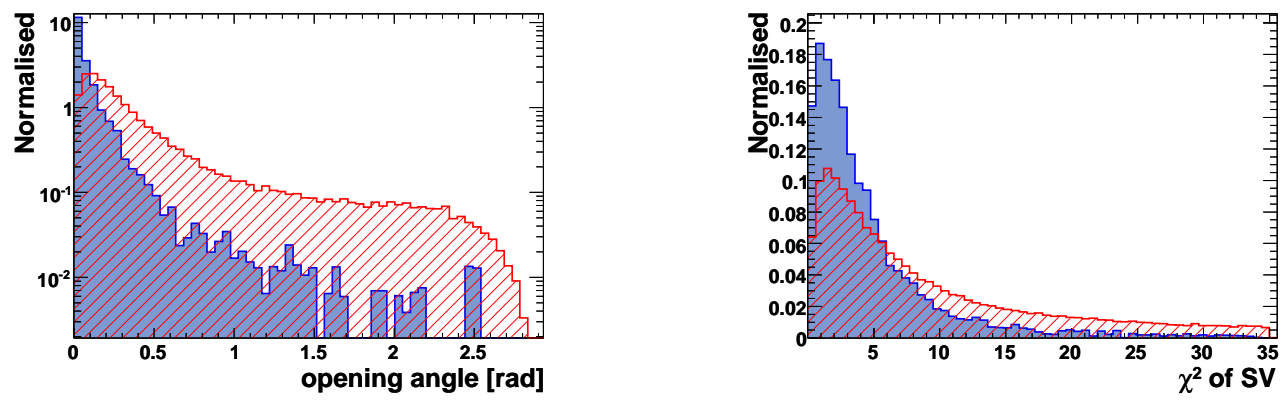

Figure 8.15: The opening angle between the decay length and the momentum of the B is shown on the left side. On the right the vertex $\chi^{2}$ can be seen. The filled histogram is the signal and the shaded histogram is the background.
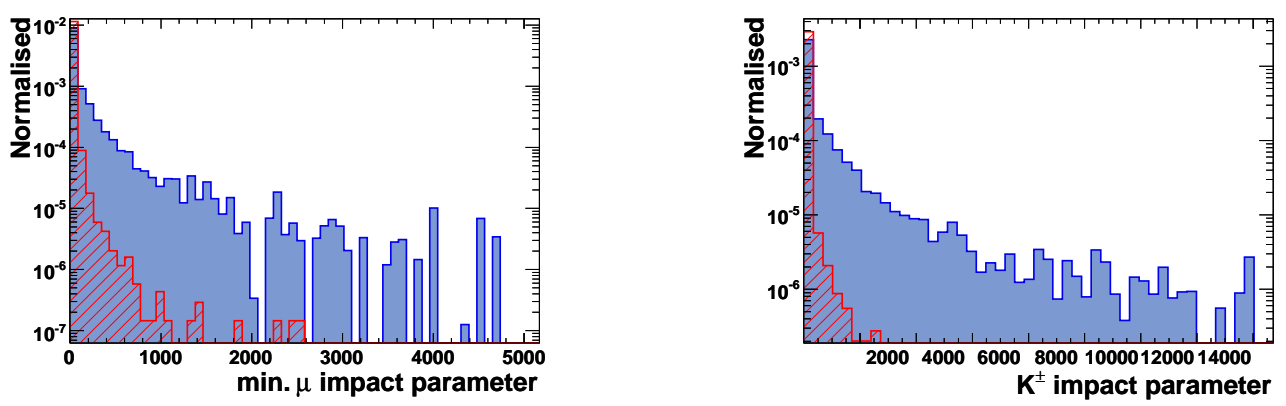

Figure 8.16: The discriminating variable minimal muon impact parameter is shown on the left and the kaon impact parameter significance on right side. The filled histogram is the signal and the shaded histogram is the background. 
As MVA selection method we have chosen a Boosted Decision Tree (BDT). The discriminating variables are tested to find the best separation between signal and background. Fig. 8.17 shows the distribution of the final selection variable of the BDT.
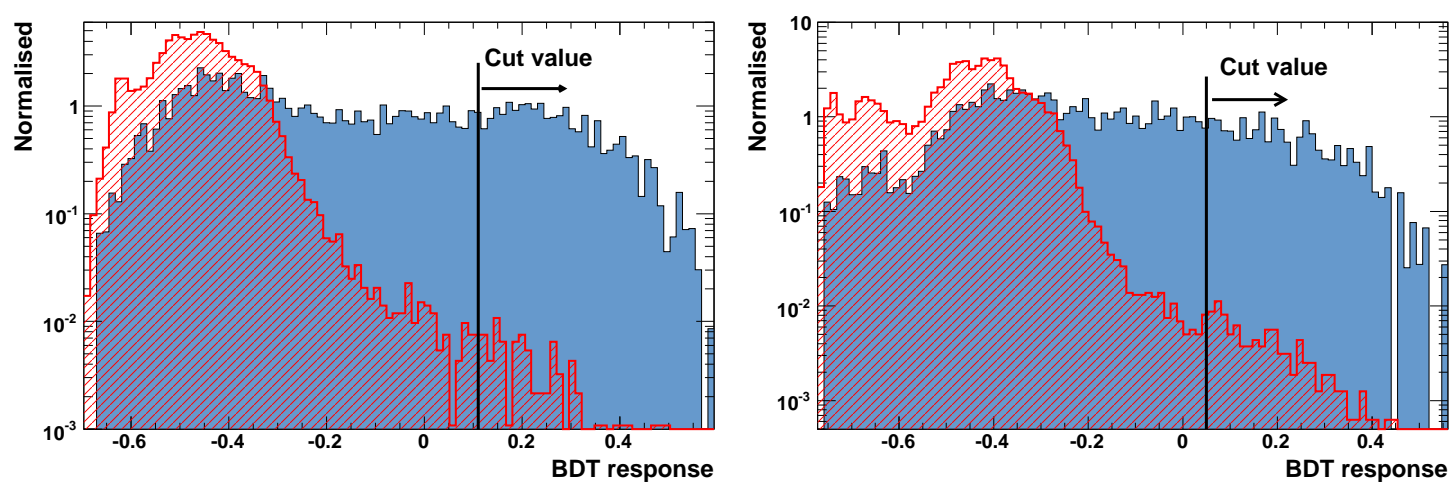

Figure 8.17: Distribution of the Boosted Decision Tree for signal (filled) and background (shaded). The plot on the left is for Run IIa and p17 Monte Carlo and the plot on the right is Run IIb data and p20 Monte Carlo.

The optimization criterion of Punzi is used to derive the best cut on the BDT response. The Punzi variable $P$ is defined as

$$
P=\frac{\epsilon_{\text {sig }}}{a / 2+\sqrt{N_{\text {back }}}}
$$

The parameter $\epsilon_{\text {sig }}$ stands for the reconstruction efficiency of the signal which is determined from Monte Carlo, $N_{b a c k}$ is the expected number of background events interpolated from the sidebands and $a$ is a constant and has to be chosen depending on the number of standard deviations corresponding to the confidence level at which the signal hypothesis is tested. For example $a=2$ corresponds to a $95 \%$ C.L. and $a=5$ corresponds to a $90 \%$ C.L. For this analysis $a=2$ was chosen.

To obtain the best cut value on the BDT response a scan of all BDT values has been performed. $N_{b a c k}$ was determined by an exponential distribution, fitted to the data using the upper and lower sidebands without taking the events in the signal window into account. Fig. 8.18 shows $P$ and the Monte Carlo selection efficiency as a function of the cut value on the BDT response. The best cut value on the BDT response for Run IIa data was obtained at 0.11 and for Run IIb data at 0.05 . All events on the right side of the cut value were used in the following section. 

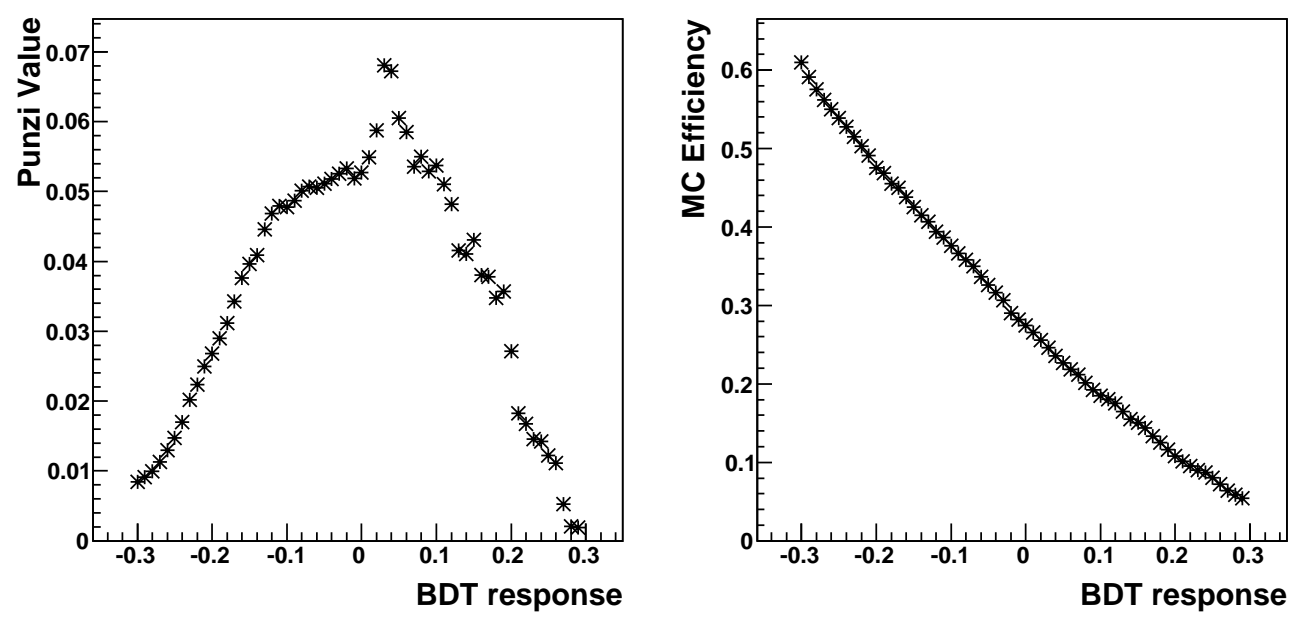

Figure 8.18: The Punzi value $P$ (left) and the signal selection efficiency $\epsilon_{\text {sig }}$ (right) as a function of the BDT response cut, as determined on Monte Carlo with all weighting functions applied. The data is from Run IIb and the Monte Carlo is p20.

\subsection{Results}

\subsubsection{Branching fraction for $B^{ \pm} \rightarrow K^{ \pm} \mu^{+} \mu^{-}$}

The Figures. 8.19 and 8.20 show the invariant mass of the reconstructed $\mu \mu K$ candidates after applying the BDT-cut at 0.11 (Run IIa) and 0.05 (Run IIb) on the full data sample as described in the previous sections. In total 25 (Run IIa) and 19 (Run IIb) events are observed in the $400 \mathrm{MeV} / \mathrm{c}^{2}$ wide signal window around the $B^{ \pm}$mass, corresponding to $\pm 2 \sigma$ of the $B^{ \pm}$mass resolution. The number of background events in the signal region was estimated by an exponential fit to the lower and upper sidebands in the $\mu \mu K$ mass plot as defined in Fig. 8.13. The fit is interpolated into the signal region and the number of background events is determined from the integral over this function within the signal region to be $16.0 \pm 4.0$ (stat) for Run IIa and $7.5 \pm 2.7$ (stat) for Run IIb. For the signal region a single Gaussian is added to the exponential distribution leading to:

$$
f(x)=\exp (a+b \cdot x)+c \cdot \exp \left(-\frac{\left(x-5.279 \mathrm{GeV} / \mathrm{c}^{2}\right)^{2}}{\left(0.1 \mathrm{GeV} / \mathrm{c}^{2}\right)^{2}}\right)
$$

For the fit the width and the mean of the signal are kept at their fixed values. Integrating the fitted function over the signal window in Fig. 8.19 and Fig. 8.20 gives $7.8 \pm 5.0$ (stat) signal events for Run IIa and 10.7 \pm 4.4 (stat) signal events for Run IIb. 


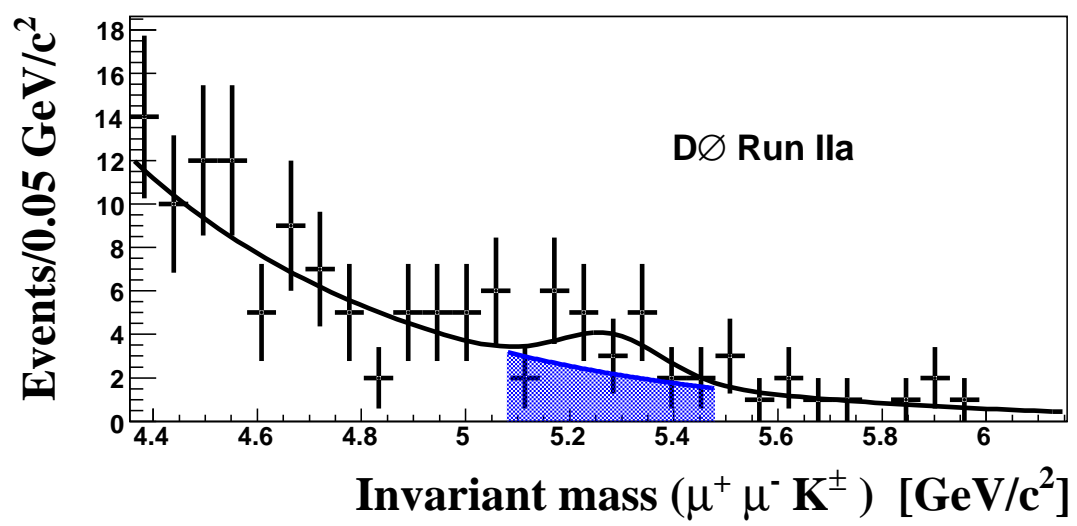

Figure 8.19: Reconstructed $\mathrm{B}^{ \pm}$mass for the decay $B^{ \pm} \rightarrow K^{ \pm} \mu^{+} \mu^{-}$in Run IIa data. The $\pm 2 \sigma$ signal window corresponds to the shaded area.

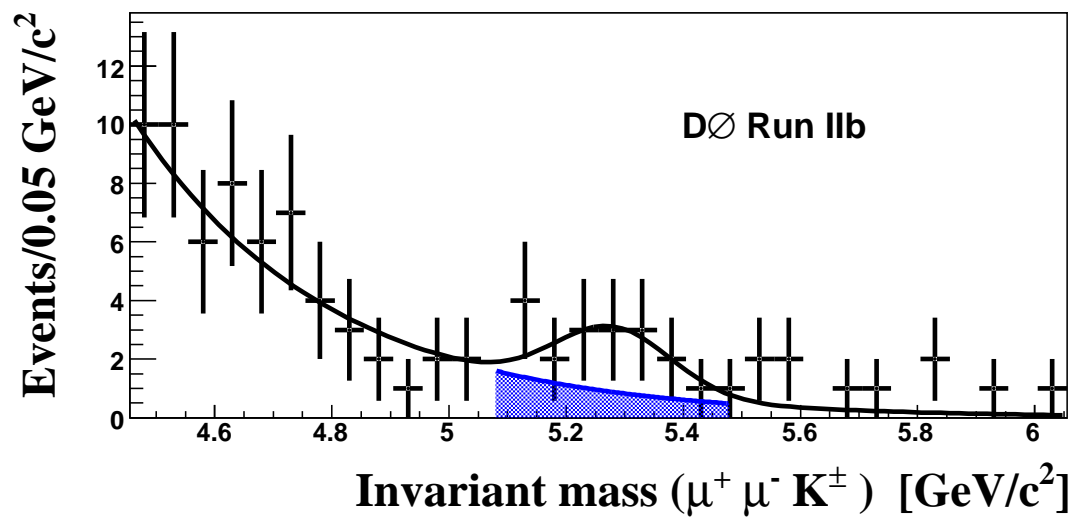

Figure 8.20: Reconstructed $\mathrm{B}^{ \pm}$mass for the decay $B^{ \pm} \rightarrow K^{ \pm} \mu^{+} \mu^{-}$in Run Ilb data. The $\pm 2 \sigma$ signal window corresponds to the shaded area.

Since the number of signal events is small, we attempt to derive an upper limit for the branching fraction $\mathcal{B}\left(B^{ \pm} \rightarrow K^{ \pm} \mu^{+} \mu^{-}\right)$according to Equation 2.16 replacing $N_{\text {sig }}$, the number of signal events, by $N_{\text {lim }}$, the upper limit.

$$
\mathcal{B}\left(B^{ \pm} \rightarrow K^{ \pm} \mu^{+} \mu^{-}\right)=\frac{N_{\text {lim }}}{N_{\text {Norm }}} \cdot \frac{\epsilon_{\text {Norm }}}{\epsilon_{\text {Signal }}} \cdot \mathcal{B}\left(B^{ \pm} \rightarrow J / \psi K^{ \pm}\right) \cdot \mathcal{B}\left(J / \psi \rightarrow \mu^{+} \mu^{-}\right)
$$

$N_{\text {lim }}$ can be calculated using a program based on a frequentist (Feldman-Cousins) approach which includes a proper treatment of the systematic errors described by Gaussians [102, 103, 104].

In Table 8.3 all sources of the uncertainties on the branching fraction $\mathcal{B}\left(B^{ \pm} \rightarrow\right.$ $K^{ \pm} \mu^{+} \mu^{-}$) calculation can be found. The dominating uncertainty of about $65.4 \%$ (Run 
Rare Decay $B^{ \pm} \rightarrow K^{ \pm} \mu^{+} \mu^{-}$

IIa) and $40.7 \%$ (Run IIb) originates from the statistics of the small number of observed events. The systematic uncertainty on the BDT method was estimated by varying the cut value on the BDT response and observing the resulting variation of the branching fraction. The uncertainty on the Monte Carlo weighting is determined by determing the branching fraction with and without the $p_{t}^{B}$-weight. The analysis was performed with loose, medium or tight quality muons and the differences of the resulting branching fractions were used as the systematical error due to the muon quality. The branching fractions $\mathcal{B}\left(J / \psi \rightarrow \mu^{+} \mu^{-}\right)=(5.88 \pm 0.1) \times 10^{-2}$ and $\mathcal{B}\left(B^{ \pm} \rightarrow J / \psi K^{ \pm}\right)=(1.0 \pm 0.04) \times 10^{-3}$ are taken from the PDG [14].

Table 8.3: The sources of the relative uncertainties of the branching fraction calculation.

\begin{tabular}{lcc}
\hline \hline Data & Run IIa & Run IIb \\
\hline Source & relative uncertainty [\%] & relative uncertainty [\%] \\
\hline Number of $B^{ \pm} \rightarrow K^{ \pm} \mu^{+} \mu^{-}$events & 65.4 & 40.7 \\
Number of $B^{ \pm} \rightarrow J / \psi K^{ \pm}$events & 2.5 & 2.7 \\
Efficiency ratio $\epsilon_{\text {Norm }} / \epsilon_{\text {Signal }}$ & negligible & negligible \\
\hline Total statistical uncertainty & 65.4 & 40.8 \\
\hline \hline Monte Carlo weighting & 8.0 & 5.8 \\
BDT method & 12.5 & 8.3 \\
Muon quality & 14.2 & 12.5 \\
$\mathcal{B}\left(J / \psi \rightarrow \mu^{+} \mu^{-}\right)$ & 1.7 & 1.7 \\
$\mathcal{B}\left(B^{ \pm} \rightarrow J / \psi K^{ \pm}\right)$ & 4.0 & 4.0 \\
\hline Total systematic uncertainty & 21.0 & 16.7 \\
\hline \hline
\end{tabular}

Together with the total of 25 (19) observed events in the $400 \mathrm{MeV} / c^{2}$ wide signal region this corresponds to an upper limit of $N_{l i m}=23.6$ including a $21.0 \%$ systematical uncertainty (see Table 8.3) for Run IIa and $N_{l i m}=24.3$ including a $16.7 \%$ systematical uncertainty for Run IIb.

The mass resolution of the $B^{ \pm} \rightarrow J / \psi K^{ \pm}$is better due to the mass constraint on the $J / \psi$ and is found to be $45 \mathrm{MeV} / c^{2} . N_{\text {norm }}$ is the number of reconstructed events of the normalization channel $B^{ \pm} \rightarrow J / \psi K^{ \pm}$. For the normalization channel $B^{ \pm} \rightarrow J / \psi K^{ \pm}$ similar cuts as specified in Table 8.1 were applied and the same MVA was performed. The background of the invariant $J / \psi K$ mass was described best by a second order polynomial distribution and for the signal a single Gaussian is added (see Fig. 8.21). The fit to the Run 
IIa data results in $1577 \pm 40$ (stat) events and that to the Run IIb data in $1614 \pm 44$ (stat) events. The number of events from the signal channel in the normalisation channel is negligible.
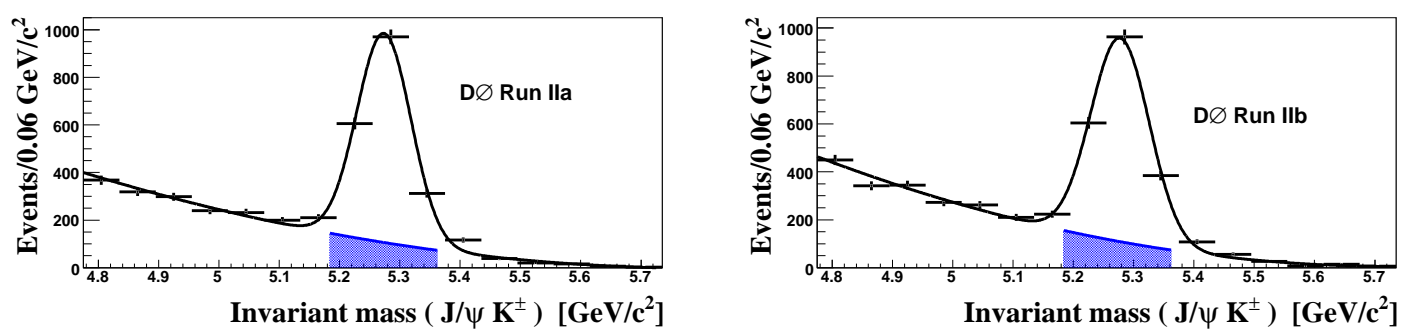

Figure 8.21: Reconstructed $B^{ \pm}$mass for the decay $B^{ \pm} \rightarrow J / \psi K^{ \pm}$in Run IIa (left) and Run IIb (right) data. The shaded area highlights a $\pm 2 \sigma$ range, where $\sigma=45 \mathrm{MeV} / \mathrm{c}$.

The efficiencies $\epsilon_{\text {Signal }}$ and $\epsilon_{\text {Norm }}$ for the signal and normalization channel are determined from Monte Carlo simulated events, including all selection cuts and corrections, resulting in $\epsilon_{\text {Norm }} / \epsilon_{\text {Signal }}=2.09 \pm 0.17$ (syst) for Run IIa and $1.70 \pm 0.10$ (syst) for Run IIb. The systematic uncertainty includes the effect of weighting the Monte Carlo data, as described in Section 8.2. The statistical uncertainty on the efficiency ratio is negligible.

As a result the upper limit for the branching fraction for $B^{ \pm} \rightarrow K^{ \pm} \mu^{+} \mu^{-}$according to Equation 8.7 and including the systematic uncertainties listed in Table 8.3 is

$$
\begin{array}{ll}
\mathcal{B}\left(B^{ \pm} \rightarrow K^{ \pm} \mu^{+} \mu^{-}\right) \leq 18.4 \times 10^{-7} & \text { Run IIa } \\
\mathcal{B}\left(B^{ \pm} \rightarrow K^{ \pm} \mu^{+} \mu^{-}\right) \leq 15.0 \times 10^{-7} & \text { Run IIb }
\end{array}
$$

at $95 \% \mathrm{CL}$.

In addition, we tried to estimate the actual branching fraction from the number of observed signal events. To calculate the branching fraction $N_{\text {lim }}$ in Equation 8.7 is replaced by the number of signal events from the fit. Including all uncertainties the branching fraction is

$$
\begin{array}{ll}
\mathcal{B}\left(B^{ \pm} \rightarrow K^{ \pm} \mu^{+} \mu^{-}\right)=6.07 \pm 3.96(\text { stat }) \pm 1.27(\text { syst }) \times 10^{-7} & \text { Run IIa } \\
\mathcal{B}\left(B^{ \pm} \rightarrow K^{ \pm} \mu^{+} \mu^{-}\right)=6.63 \pm 2.71(\text { stat }) \pm 1.11(\text { syst }) \times 10^{-7} & \text { Run IIb }
\end{array}
$$

The combination of Run IIa and Run IIb results in the final branching fraction of

$$
\mathcal{B}\left(B^{ \pm} \rightarrow K^{ \pm} \mu^{+} \mu^{-}\right)=6.45 \pm 2.24 \text { (stat) } \pm 1.19 \text { (syst) } \times 10^{-7}
$$

Within the errors this measurement is in agreement with the SM prediction (see Table 2.2) and previous measurements of other experiments (see Table 2.3).

An overview of all numbers entering the branching fraction calculation is given in Table 8.4. 
Table 8.4: The values for the different variables of Equation 8.7 for different BDT cut values without their errors.

\begin{tabular}{l|c|c}
\hline \hline & Run IIa & Run IIb \\
\hline Variable & BDT cut 0.11 & BDT cut 0.05 \\
\hline \hline$N_{\text {lim }}\left(N_{\text {sig }}\right)$ & $7.8(23.6)$ & $10.7(24.3)$ \\
\hline$N_{\text {norm }}$ & 1577 & 1614 \\
\hline$N_{J / \psi}^{\text {MonteCarlo }}$ & 2369 & 1014 \\
\hline$N_{\mu \mu}^{M o n t e C a r l o}$ & 1303 & 721 \\
\hline$\epsilon_{\text {Norm }} / \epsilon_{\text {Signal }}$ & 2.09 & 1.70 \\
\hline Branching fraction & $6.07 \cdot 10^{-7}$ & $6.63 \cdot 10^{-7}$ \\
\hline \hline
\end{tabular}




\section{Chapter 9}

\section{The decay $B_{d}^{0} \rightarrow K^{*} \mu^{+} \mu^{-}$}

This Chapter describes the search for the rare decay of $B_{d}^{0} \rightarrow K^{*} \mu^{+} \mu^{-}$. As normalisation channel $B_{d}^{0} \rightarrow J / \psi K^{*}(892)$ is used with $J / \psi \rightarrow \mu^{+} \mu^{-}$. The same trigger-conditions as described in chapter 8 are used and the strategy of the analysis is the same. For the final selection the BDT method is used.

\subsection{Introduction}

There are several similarities between the decay channel $B_{d}^{0} \rightarrow K^{*} \mu^{+} \mu^{-}$and $B^{ \pm} \rightarrow$ $K^{ \pm} \mu^{+} \mu^{-}$.
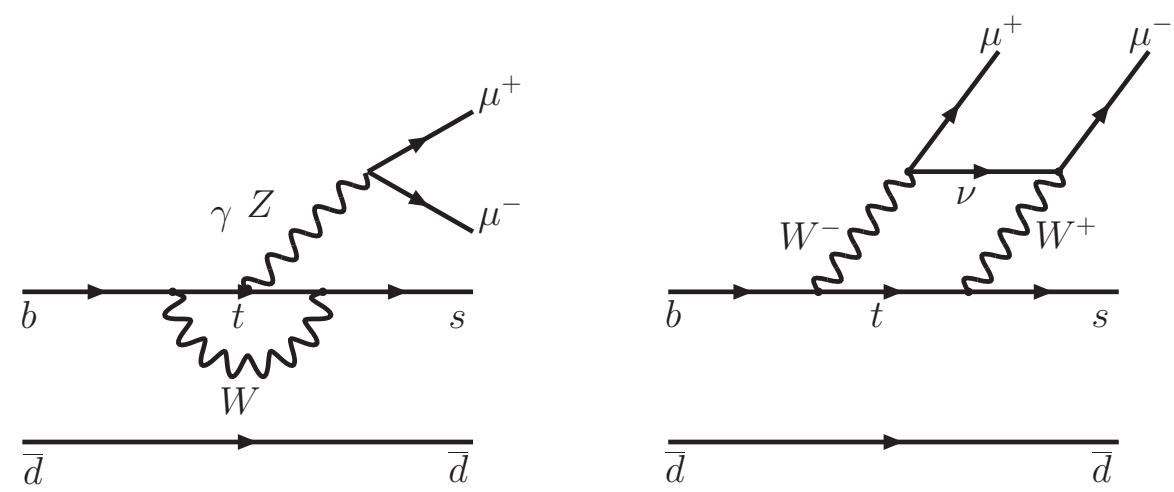

Figure 9.1: SM diagrams for the decay $B_{d}^{0} \rightarrow K^{*} \mu^{+} \mu^{-}$.

The dimuon spectra is very similar and in the data there are the same resonances of $J / \psi$ or a $\psi$ as for the decay $B^{ \pm} \rightarrow K^{ \pm} \mu^{+} \mu^{-}$. The main difference is that the final state consists of four tracks. The $K^{*}$ decays into a kaon and a pion. As the D $\varnothing$ detector does 
not provide a particle identification one has to find correct assignments for the third and fourth particle. For each event exists a list of all the particles ordered by their $p_{t}$. In the reconstruction the $K^{*}$ candidates are built of the combination of all the particles in the particle list except the muons. Then the masses of the kaon and the pion are assigned to the two decay particles of the $K^{*}$ to calculate the invariant mass of the $K^{*}$. The mass of the $K^{*}$ tends to be shifted if the kaon mass is assigned to the pion and vice versa (see Fig. 9.2). $K^{*} \rightarrow \pi K$ is used when the pion has the higher momenta then the kaon and $K^{*} \rightarrow K \pi$ is used when the kaon has the higher $p_{t}$ than the pion. The effect of assigning the wrong mass to the particle with the higher $p_{t}$ is smaller than for the low $p_{t}$ particle. By assigning a smaller mass to the low $p_{t}$ particle, the final mass will be shifted to lower values what can be seen in the left plot of Fig. 9.2.
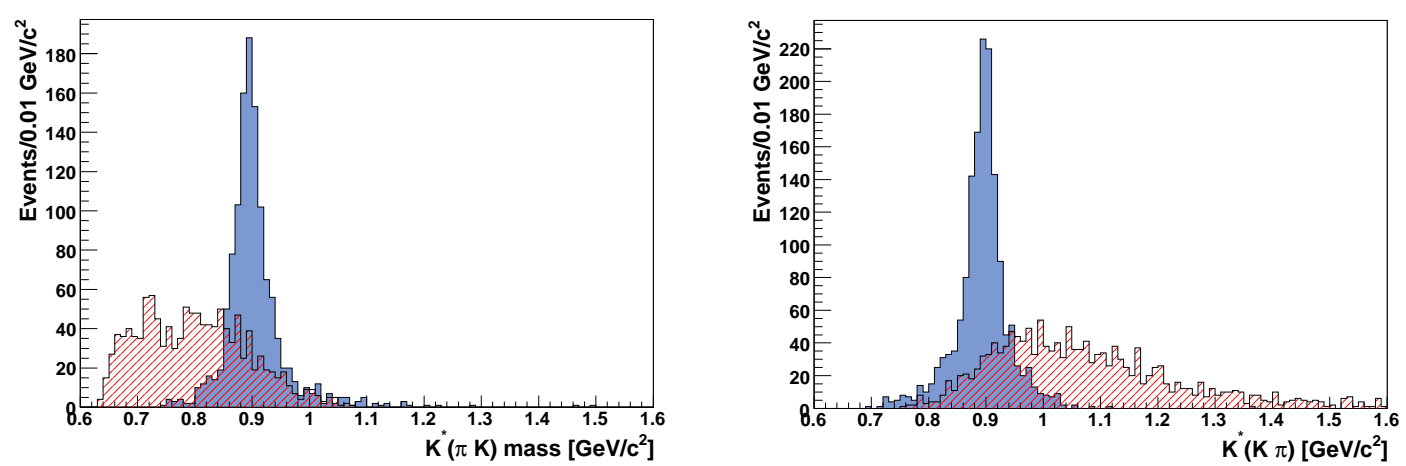

Figure 9.2: Invariant mass distributions of the $(K \pi))$ pair. The plot on the left is for the case that the higher momentum particle is the pion, the plot on the right is for the case where the higher momentum particle is the kaon. The true combination is the filled distribution and if the wrong masses assigned to the decay products (shaded). On the right side the same is shown for the channel $K^{*} \rightarrow K \pi$.

\subsection{Pre-selection}

The pre-selection cuts for the decay $B_{d}^{0} \rightarrow K^{*} \mu^{+} \mu^{-}$were very similar to those used for the $B^{ \pm} \rightarrow K^{ \pm} \mu^{+} \mu^{-}$analysis. For the two muons the same cuts were applied as described in section 8.2. The $K^{*}$ decays into a $K^{ \pm}$and a $\pi^{\mp}$ and it is required that both particles have a $p_{t}>0.5 \mathrm{GeV} / \mathrm{c}$ and at least one hit in the SMT detector. The $K^{*}$ resolution in Monte Carlo is about $25 \mathrm{MeV} / \mathrm{c}^{2}$ as shown in Fig. 9.3. The $J / \psi$ and the $\psi(2 s)$ resonances were cut out from the dimuon mass spectrum $\left(2.5 \mathrm{GeV} / \mathrm{c}^{2}<m_{\mu^{+} \mu^{-}}<4.04 \mathrm{GeV} / \mathrm{c}^{2}\right)$. To further reduce background, a cut on the reconstructed $K^{*}$ mass of $0.812 \mathrm{GeV} / \mathrm{c}^{2}<m_{K^{*}}<0.967 \mathrm{GeV} / \mathrm{c}^{2}$ was applied corresponding 

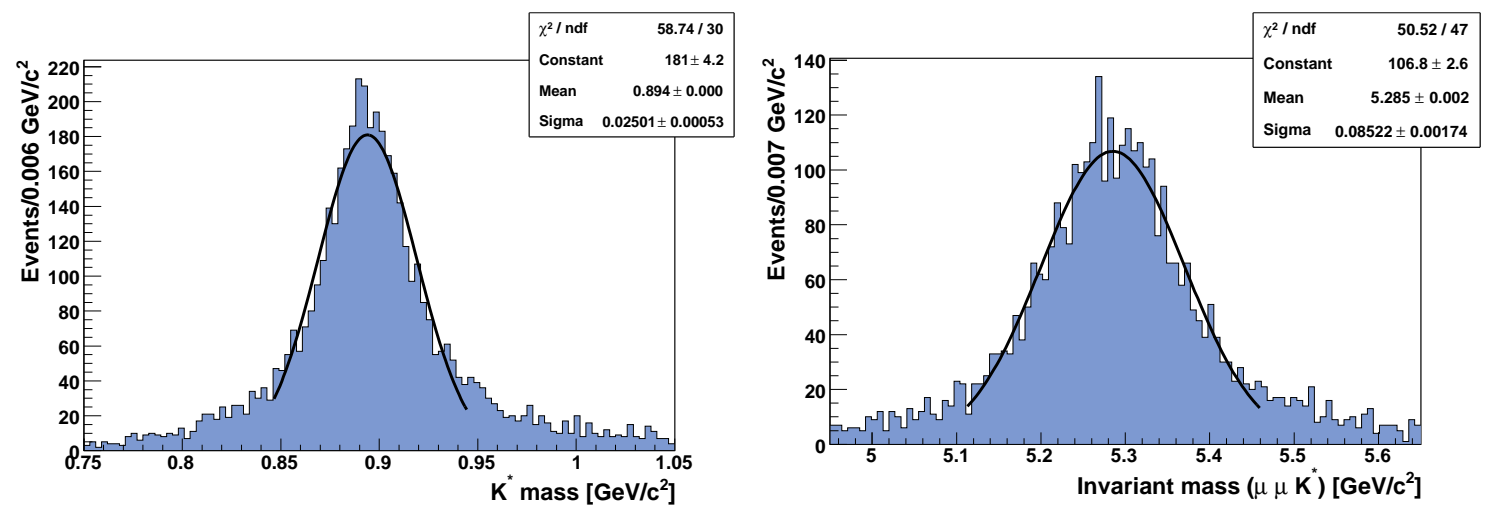

Figure 9.3: $K^{*}$ mass resolution (left) and the $B_{d}$ mass resolution (right) determined by Monte Carlo.

to $\pm 3 \sigma$. In case of multiple $B_{d}^{0}$ candidates, the one with highest transverse momentum of the $K^{*}$ was selected. The list of all pre-selection cuts is shown in Table 9.1.

Table 9.1: Preselection cuts for the $B_{d}^{0} \rightarrow K^{*} \mu^{+} \mu^{-}$candidates. For the calibration channel $B_{d}^{0} \rightarrow J / \psi K^{*}(892)$ the same cuts are applied except for the invariant $\mu \mu$ mass where the range $2.84 \mathrm{GeV} / \mathrm{c}^{2}<m_{\mu^{+} \mu^{-}}<3.35 \mathrm{GeV} / \mathrm{c}^{2}$ is selected.

\begin{tabular}{l|c}
\hline \hline Cuts & value \\
\hline \hline muon quality & medium (2) \\
\hline sum of muon charge & 0 \\
\hline muon $p_{t}^{\mu}$ & $p_{t}^{\mu} \geq 2.5 \mathrm{GeV} / \mathrm{c}$ \\
\hline pseudo rapidity of muons & $\left|\eta_{\mu}\right| \leq 2.0$ \\
\hline muon tracking hits & $2 \mathrm{SMT}, 2 \mathrm{CFT}$ \\
\hline third and fourth particle & $p_{t}>0.5 \mathrm{GeV} / \mathrm{c} ; 1 \mathrm{SMT} \mathrm{hit}$ \\
\hline excluded mass window & $2.50 \mathrm{GeV} / \mathrm{c}^{2}<\mathrm{M} \mu_{\mu}<4.04 \mathrm{GeV} / \mathrm{c}^{2}$ \\
\hline mass window of $K^{*}$ & $0.816 \mathrm{GeV} / \mathrm{c}^{2}<m_{K^{*}}<0.966 \mathrm{GeV} / \mathrm{c}^{2}$ \\
\hline Vertex $\chi^{2}$ & $\chi^{2}<35$ \\
\hline best candidate & highest $p_{t}^{K^{*}}$ \\
\hline \hline
\end{tabular}

As the same trigger was applied as for $B^{ \pm} \rightarrow K^{ \pm} \mu^{+} \mu^{-}$the values for the Monte 
Carlo trigger simulation were taken from section 8.3 and just the weight depending on the $p_{t}^{B}$ was newly determined and can be seen in Fig. 9.4. The $p_{t}^{B}$ spectrum before and after applying the $p_{t}^{B}$ weights can be seen in Fig. 9.5. Fig. 9.6 shows the $p_{t}$ distributions of the muons in Monte Carlo and data after applying the weight-functions. Due to this reweighting procedure a satisfactory agreement between data and Monte Carlo could be obtained.

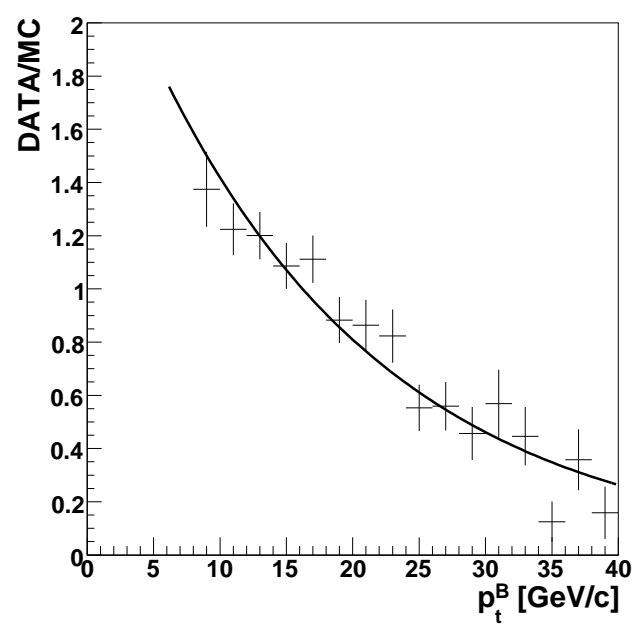

Figure 9.4: Weighting function determined from the ratio from $p_{t}^{B}$ data (Run IIb) over p20 Monte Carlo for the calibration channel $B_{d}^{0} \rightarrow J / \psi K^{*}(892)$.

For the further analysis the $B_{d}^{0}$ mass resolution in data has to be determined. Equation 8.1 was used and a mass resolution of about $93 \mathrm{MeV} / c^{2}$ was obtained for the decay $B_{d}^{0} \rightarrow K^{*} \mu^{+} \mu^{-}$.

\subsection{Final selection - TMVA}

To obtain the best possible distinction between signal and background a similar analysis as in section 8.4 was performed. In addition to the variables described in section 7.4 the impact parameter of the additional particle was included. The following variables are used in the MVA:

- Transverse decay length significance (see Fig. 9.7)

- Isolation variable (see Fig. 9.7) 

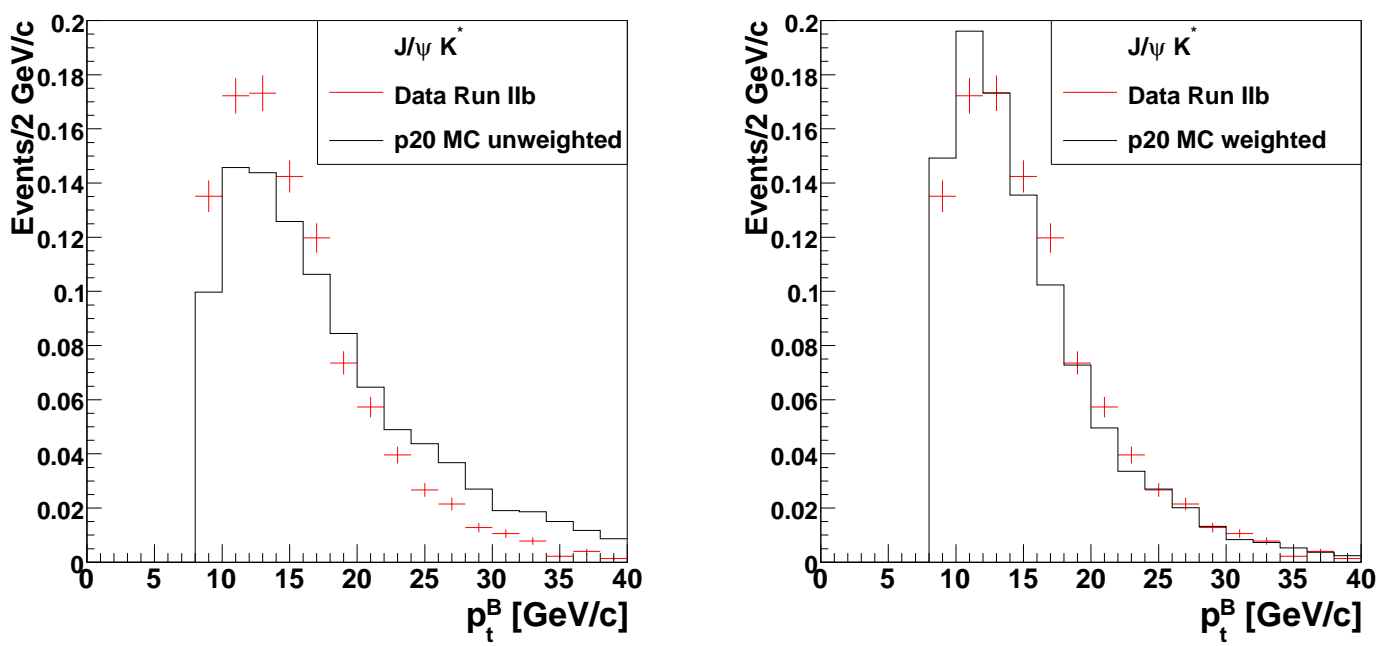

Figure 9.5: $p_{t}^{B}$ distributions for the $B_{d}^{0} \rightarrow J / \psi K^{*}(892)$ calibration channel. The Monte Carlo distribution (line) is compared to the preselected data of Run IIb (crosses) before (left) and after (right) applying the $p_{t}$ reweighting function.
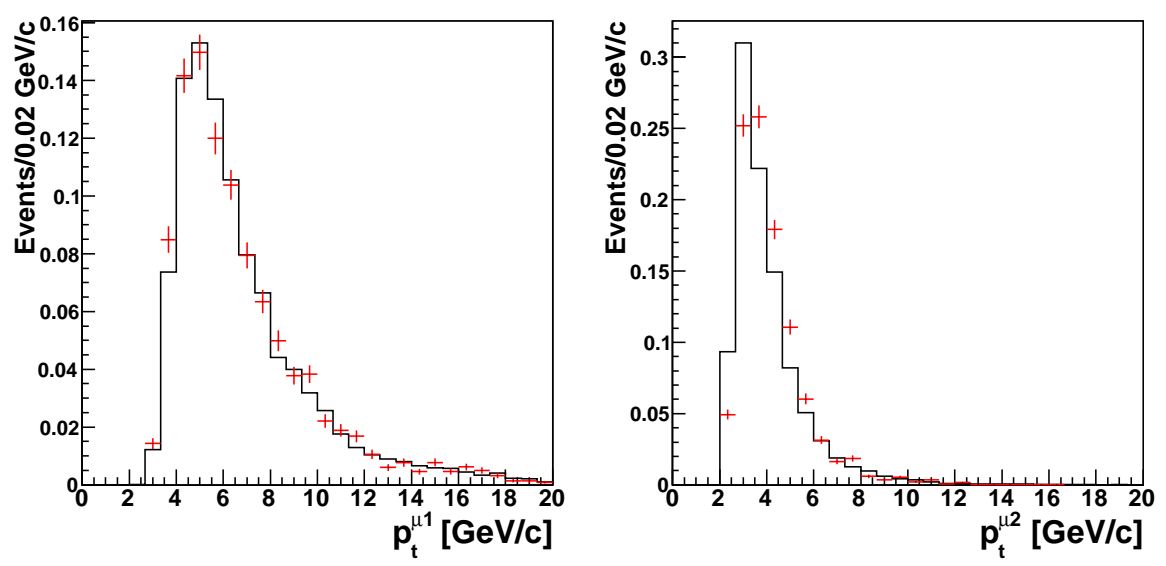

Figure 9.6: The $p_{t}^{\mu 1}$ (left) and $p_{t}^{\mu 2}$ (right) distributions for Run IIb data after preselection (crosses) and Monte Carlo (line), after reweighting with the trigger functions and the $p_{t}^{B}$ weighting function.

- Opening angle (see Fig. 9.8)

- Vertex quality (see Fig. 9.8)

- Muon impact parameter (see Fig.9.9) 
- Kaon impact parameter (see Fig. 9.9)

- Pion impact parameter (see Fig. 9.10)
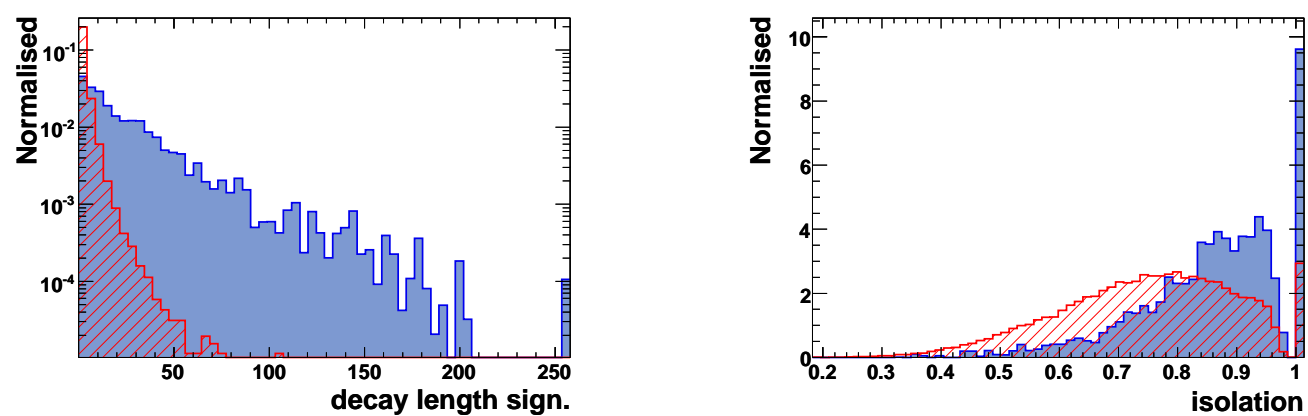

Figure 9.7: The transverse decay length significance is shown on the left. On the right the isolation variable can be seen. The filled histogram is the signal and the shaded histogram is the background.
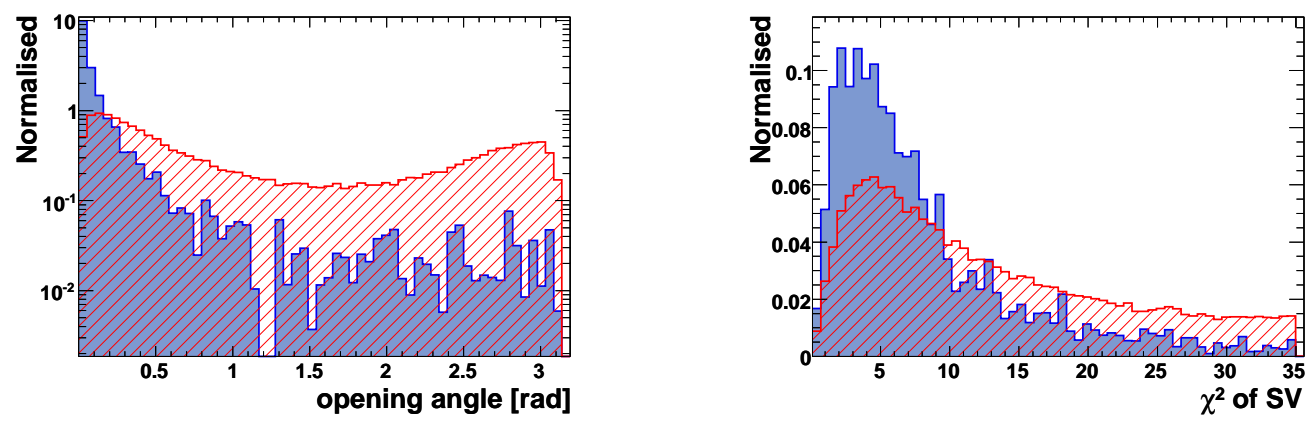

Figure 9.8: The opening angle between the decay length and the momentum of the B is shown on the left. On the right the vertex $\chi^{2}$ can be seen. The filled histogram is the signal and the shaded histogram is the background.

The sidebands for the $\mu \mu K^{*}$ mass distribution were defined from $4.379 \mathrm{GeV} / \mathrm{c}^{2}$ to $4.979 \mathrm{GeV} / \mathrm{c}^{2}$ for the upper and $5.579 \mathrm{GeV} / \mathrm{c}^{2}$ to $6.179 \mathrm{GeV} / \mathrm{c}^{2}$ ) for the lower sideband. The signal region was defined between $5.093 \mathrm{GeV} / \mathrm{c}^{2}$ and $5.465 \mathrm{GeV} / \mathrm{c}^{2}$. The BDT response for the Monte Carlo and the sidebands is shown in Fig. 9.11. The optimization criterion of Punzi (Equation 8.5) gives best cut values of -0.02 for Run IIa and 0.10 for Run IIb. 

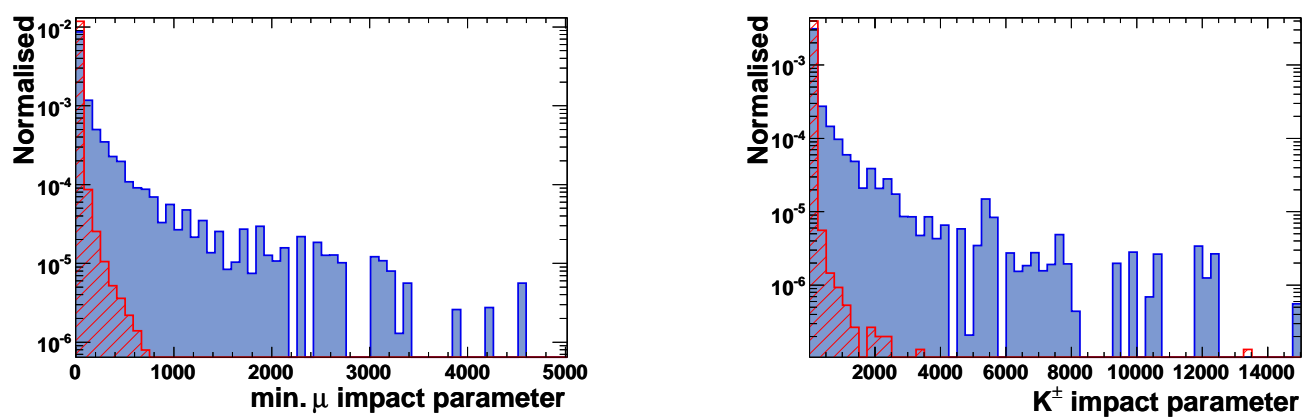

Figure 9.9: The discriminating variable minimal muon impact parameter is shown on the left and the kaon impact parameter significance on the right. The filled histogram is the signal and the shaded histogram is the background.

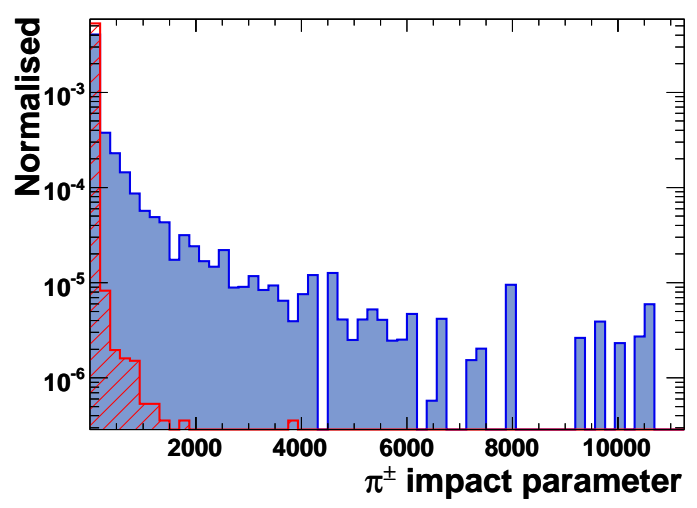

Figure 9.10: Pion impact parameter significance. The filled histogram is the signal and the shaded histogram is the background.

\subsection{Results}

The invariant mass of the reconstructed $\mu \mu K^{*}$ candidates after applying the BDT-cut at -0.02 for Run IIa, respectively at 0.10 for Run IIb, is shown in the Fig. 9.12 and 9.13. In total 12 (Run IIa) and 19 (Run IIb) events are observed in the $372 \mathrm{MeV} / \mathrm{c}^{2}$ signal window, corresponding to 2 standard deviations of the $B_{d}^{0}$ mass resolution.

As described in section 8.5 the data is fitted with an exponential describing the background and a Gaussian describing the signal.

$$
f(x)=\exp (a+b \cdot x)+c \cdot \exp \left(-\frac{\left(x-5.279 \mathrm{GeV} / \mathrm{c}^{2}\right)^{2}}{\left(0.093 \mathrm{GeV} / \mathrm{c}^{2}\right)^{2}}\right)
$$

The sidebands of the measured mass distributions were fitted with an exponential which 

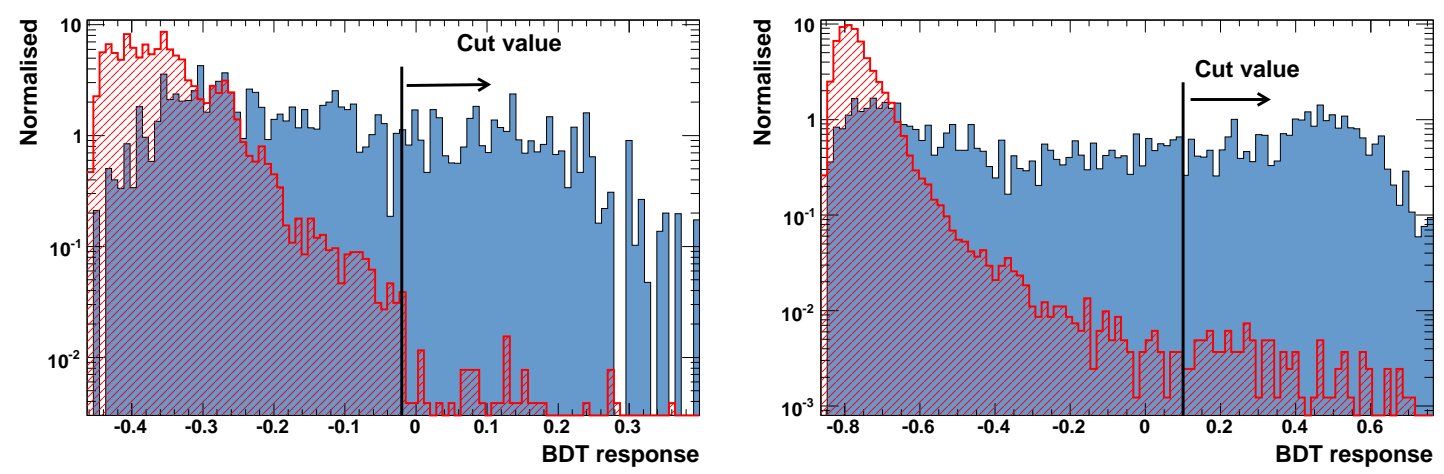

Figure 9.11: Distribution of the Boosted Decision Tree for signal (filled) and background (shaded). The plot on the left is for Run IIa and p17 Monte Carlo and the plot on the right is Run IIb data and p20 Monte Carlo.

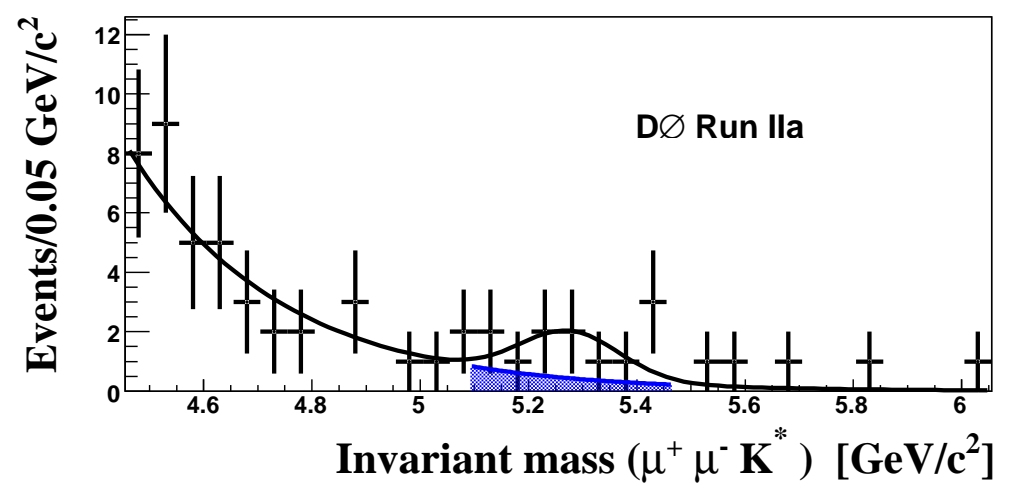

Figure 9.12: Reconstructed $\mathrm{B}_{d}$ mass for the decay $B_{d}^{0} \rightarrow K^{*} \mu^{+} \mu^{-}$in Run IIa data. The $\pm 2 \sigma$ range corresponds to the shaded area.

then was interpolated to the signal region. The integral of this exponential over the signal region was used to estimate the number of expected background events. This results in $3.5 \pm 1.9$ (stat) events for Run IIa and in 4.2 \pm 2.0 (stat) events for Run IIb. To obtain the number of signal events of the decay $B_{d}^{0} \rightarrow K^{*} \mu^{+} \mu^{-}$, the mass of the $B_{d}^{0}$ was fixed to the PDG value of $M_{B_{d}}=5.279 \mathrm{GeV} / \mathrm{c}^{2}$ and the width to the expected mass resolution of $\sigma_{m}=93 \mathrm{MeV} / \mathrm{c}^{2}$. The number of signal events was determined by integrating over the Gaussian in the signal region. This resulted in 7.1 13.5 (stat) signal events for Run IIa and $13.8 \pm 4.4$ (stat) signal events for Run IIb.

Since the number of signal events is small, we attempt to derive an upper limit for the branching fraction $\mathcal{B}\left(B_{d}^{0} \rightarrow K^{*} \mu^{+} \mu^{-}\right)$according to Equation 2.16 replacing $N_{\text {sig }}$, the 


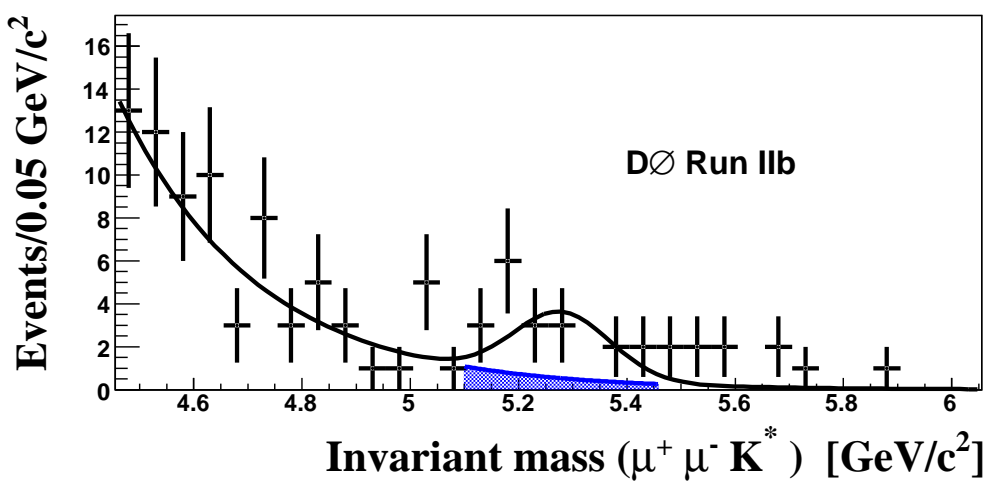

Figure 9.13: Reconstructed $\mathrm{B}_{d}$ mass for the decay $B_{d}^{0} \rightarrow K^{*} \mu^{+} \mu^{-}$in Run IIb data. The $\pm 2 \sigma$ range corresponds to the shaded area.

number of signal events, by $N_{\text {lim }}$, the upper limit.

$$
\mathcal{B}\left(B_{d}^{0} \rightarrow K^{*} \mu^{+} \mu^{-}\right)=\frac{N_{\text {lim }}}{N_{\text {Norm }}} \cdot \frac{\epsilon_{\text {Norm }}}{\epsilon_{\text {Signal }}} \cdot \mathcal{B}\left(B_{d}^{0} \rightarrow J / \psi K^{*}(892)\right) \cdot \mathcal{B}\left(J / \psi \rightarrow \mu^{+} \mu^{-}\right)
$$

Table 9.2 shows all sources of relative uncertainties on the calculation of the branching fraction $\mathcal{B}\left(B_{d}^{0} \rightarrow K^{*} \mu^{+} \mu^{-}\right)$. Due to the small number of signal events the statistical error is the dominating error contribution. The systematic uncertainty on the BDT method was estimated by choosing different BDT-cutvalues and observing the resulting variation of the branching fraction. The uncertainty of the Monte Carlo weighting was determined by measuring the branching fraction with and without the Monte Carlo weighting. The systematic error of the muon quality was assumed to be the same as for $B^{ \pm} \rightarrow K^{ \pm} \mu^{+} \mu^{-}$. The errors on the branching fraction $\mathcal{B}\left(J / \psi \rightarrow \mu^{+} \mu^{-}\right)$and $\mathcal{B}\left(B_{d}^{0} \rightarrow J / \psi K^{*}(892)\right)$ were taken from the PDG [14].

Fig. 9.14 shows the invariant mass for the normalisation channel. The background was described by a second order polynomial and the signal by a Gaussian. The fit to the Run IIa data results in $1253 \pm 35$ (stat) events, the fit for the Run IIb data in $1854 \pm 47$ (stat) events.

The same approach as described in section 8.5 is used to calculate $N_{\text {lim }}$ using the program pole $[102,103,104]$. The upper limit of $N_{\text {lim }}=19.3$ including the systematic uncertainty of $18.9 \%$ for Run IIa and $N_{\text {lim }}=28.3$ including the systematic uncertainty of $16.6 \%$ for Run IIb were calculated. This results in the upper limits for the decay $B_{d}^{0} \rightarrow K^{*} \mu^{+} \mu^{-}$including the systematic uncertainties of:

$$
\begin{array}{ll}
\mathcal{B}\left(B_{d}^{0} \rightarrow K^{*} \mu^{+} \mu^{-}\right) \leq 24.4 \times 10^{-7} & \text { Run IIa } \\
\mathcal{B}\left(B_{d}^{0} \rightarrow K^{*} \mu^{+} \mu^{-}\right) \leq 26.8 \times 10^{-7} & \text { Run IIb }
\end{array}
$$


Table 9.2: The sources of the relative uncertainties of the branching fraction calculation.

\begin{tabular}{lcc}
\hline \hline Data & Run IIa & Run IIb \\
\hline Source & relative uncertainty [\%] & relative uncertainty [\%] \\
\hline Number of $B_{d}^{0} \rightarrow K^{*} \mu^{+} \mu^{-}$events & 49.3 & 31.9 \\
Number of $B_{d}^{0} \rightarrow J / \psi K^{*}(892)$ events & 2.8 & 2.5 \\
Efficiency ratio $\epsilon_{N \text { Norm }} / \epsilon_{\text {Signal }}$ & negligible & negligible \\
\hline Total statistical uncertainty & 49.4 & 32.0 \\
\hline \hline Monte Carlo weighting & 5.7 & 3.3 \\
BDT method & 9.9 & 9.3 \\
Muon quality & 14.2 & 12.5 \\
$\mathcal{B}\left(J / \psi \rightarrow \mu^{+} \mu^{-}\right)$ & 1.7 & 1.7 \\
$\mathcal{B}\left(B_{d}^{0} \rightarrow J / \psi K^{*}(892)\right)$ & 4.5 & 4.5 \\
\hline Total systematic uncertainty & 18.9 & 16.6 \\
\hline \hline
\end{tabular}
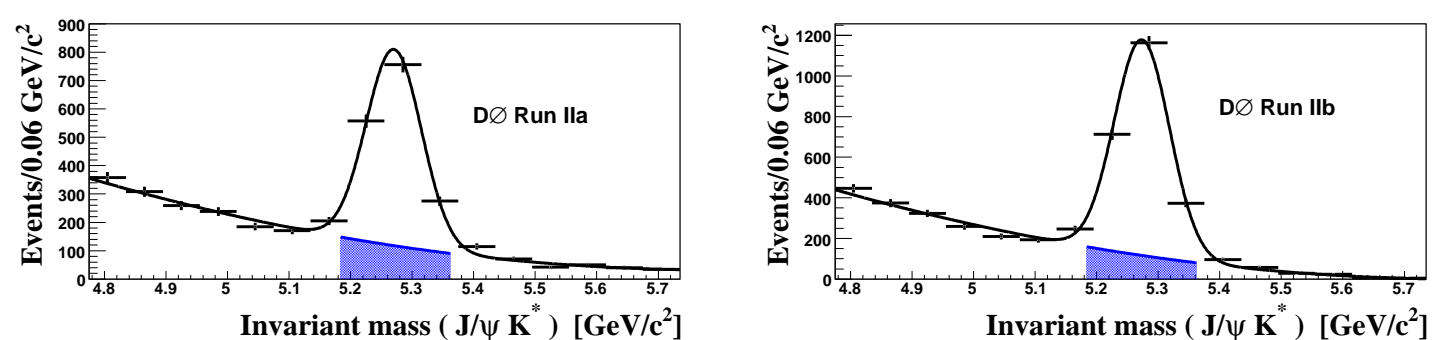

Figure 9.14: Reconstructed $\mathrm{B}_{d}$ mass for the decay $B_{d}^{0} \rightarrow J / \psi K^{*}(892)$ in Run IIa (left) and Run IIb (right) data. The shaded area highlights a $\pm 2 \sigma$ range, where $\sigma=45 \mathrm{MeV} / \mathrm{c}$.

at a $95 \% \mathrm{CL}$.

By replacing $N_{\text {lim }}$ with the number of signal events obtained from the fit (Equation 9.1) and with Equation 9.2 the branching fraction is determined:

$$
\begin{aligned}
& \mathcal{B}\left(B_{d}^{0} \rightarrow K^{*} \mu^{+} \mu^{-}\right)=8.98 \pm 4.44(\text { stat }) \pm 1.70(\text { syst }) \times 10^{-7} \quad \text { Run IIa } \\
& \mathcal{B}\left(B_{d}^{0} \rightarrow K^{*} \mu^{+} \mu^{-}\right)=13.08 \pm 4.19(\text { stat }) \pm 2.17 \text { (syst) } \times 10^{-7} \quad \text { Run IIb }
\end{aligned}
$$

The combination of Run IIa and Run IIb results in the final branching fraction of

$$
\mathcal{B}\left(B_{d}^{0} \rightarrow K^{*} \mu^{+} \mu^{-}\right)=11.15 \pm 3.05(\text { stat }) \pm 1.94(\text { syst }) \times 10^{-7}
$$


Within the errors this measurements is in agreement with the SM prediction (see Table 2.2) and previous measurements of other experiments (see Table 2.3). 


\section{Chapter 10}

\section{Conclusion}

The data used for these analysis was collected during the August 2002 and August 2008 with a total luminosity of $4.1 \mathrm{fb}^{-1}$. In the spring 2006 a new silicon layer was installed. The data before the Layer 0 installation is know as Run IIa a luminosity of about $1.4 \mathrm{fb}^{-1}$. After the Layer 0 installation Run IIb started with a luminosity of about $2.7 \mathrm{fb}^{-1}$. Due to the different detector layouts these two dataset are analyzed separately.

For all physics analysis a well aligned detector is required. Stability studies of the software alignment algorithm were performed. It was found that the systematic uncertainties for the CFT axial and radial alignment are $25 \mu \mathrm{m}$. The position resolution for the CFT is of about $100 \mu \mathrm{m}$. For the SMT the axial alignment precision is $5 \mu \mathrm{m}$, the radial precision $7 \mu \mathrm{m}$ and the $z$ precision is $15 \mu \mathrm{m}$, where the position resolution of the SMT is about $20 \mu \mathrm{m}$.

A search for the rare decay $B^{ \pm} \rightarrow K^{ \pm} \mu^{+} \mu^{-}$was performed. By fitting a Gaussian with a fixed mean $\mu=5.279 \mathrm{GeV} / \mathrm{c}^{2}$ and width $\sigma=100 \mathrm{MeV} / \mathrm{c}^{2}$ and an exponential to the same datasets $7.8 \pm 5.0$ (stat) signal and $16.0 \pm 4.0$ (stat) background events were found for Run IIa and 10.7 \pm 4.4 (stat) signal and $4.2 \pm 2.0$ (stat) background events were found for Run IIb. This results in the branching fraction of

$$
\mathcal{B}\left(B^{ \pm} \rightarrow K^{ \pm} \mu^{+} \mu^{-}\right)=6.45 \pm 2.24(\text { stat }) \pm 1.19(\text { syst }) \times 10^{-7}
$$

This result is in agreement with the SM predictions and with previous measurements.

The similar analysis was performed for the rare decay $B_{d}^{0} \rightarrow K^{*} \mu^{+} \mu^{-}$. By performig a fit of a Gaussian with a different width $\sigma=93 \mathrm{MeV} / \mathrm{c}^{2}$ to the two datasets, $7.1 \pm 3.9$ (stat) signal events were found for Run IIa and 13.8 \pm 4.4 (stat) signal events have been found for Run IIb. The branching fraction of

$$
\mathcal{B}\left(B_{d}^{0} \rightarrow K^{*} \mu^{+} \mu^{-}\right)=11.15 \pm 3.05(\text { stat }) \pm 1.94(\text { syst }) \times 10^{-7}
$$

was calculated. The branching fraction agrees with the SM prediction. 
The Tevatron accelerator is scheduled to run until 2009, with a projected integrated luminosity of $8 \mathrm{fb}^{-1}-9 \mathrm{fb}^{-1}$. With such a large data sample the Tevatron experiments will be able to further investigations on rare decays. 


\section{Bibliography}

[1] S. Weinberg. A Model of Leptons. Phys. Rev. Lett., 19(21):1264-1266, Nov 1967.

[2] S. L. Glashow. Partial Symmetries of Weak Interactions. Nucl. Phys., 22:579-588, 1961.

[3] A. Salam. Weak and Electromagnetic Interactions. Originally printed in *Svartholm: Elementary Particle Theory, Proceedings Of The Nobel Symposium Held 1968 At Lerum, Sweden*, Stockholm 1968, 367-377.

[4] G. Altarelli. A QCD Primer, 2002.

[5] F. Halzen and A. Martin. Quark and Leptons. Wiley, 1984.

[6] Y. Fukuda et al. Evidence for Oscillation of Atmospheric Neutrinos. Phys. Rev. Lett., 81(8):1562-1567, Aug 1998.

[7] A. Pich. The Standard Model of Electroweak Interactions, 2005.

[8] J. H. Christenson, J. W. Cronin, V. L. Fitch, and R. Turlay. Evidence for the $2 \pi$ Decay of the $K_{2}^{0}$ Meson. Phys. Rev. Lett., 13(4):138-140, Jul 1964.

[9] R. Fleischer. Recent Theoretical Developments in CP Violation in the B System. NUCL.INSTRUM.METH.A, 446:1, 2000.

[10] A. J. Buras. CP Violation and Rare Decays of K and B Mesons, 1999.

[11] P. F. Harrison et al. (BaBar Collaboration). The BaBar physics book: Physics at an asymmetric B factory. Papers from Workshop on Physics at an Asymmetric B Factory (BaBar Collaboration Meeting), Rome, Italy, 11-14 Nov 1996, Princeton, NJ, 17-20 Mar 1997, Orsay, France, 16-19 Jun 1997 and Pasadena, CA, 22-24 Sep 1997.

[12] N. Cabibbo. Unitary Symmetry and Leptonic Decays. Phys. Rev. Lett., 10(12):531533, Jun 1963. 
[13] M. Kobayashi and T. Maskawa. CP-Violation in the Renormalizable Theory of Weak Interaction. Prog. Th. Phys., 1973.

[14] C. Amsler et al. Review of particle physics. Phys. Lett, B667, 2008.

[15] R. Aleksan et al. Determining the Quark Mixing Matrix from CP-violating Asymmetries. Phys. Rev. Lett., 73(1):18-20, Jul 1994.

[16] J. F. Gunion. New (and Old) Perspectives on Higgs Physics, 2008.

[17] S. L. Glashow, J. Iliopoulos, and L. Maiani. Weak Interactions with Lepton-Hadron Symmetry. Phys. Rev. D, 2(7):1285-1292, Oct 1970.

[18] A. J. Buras and R. Fleischer. Quark mixing, CP violation and rare decays after the top quark discovery. ADV.SER.DIRECT.HIGH ENERGY PHYS., 15:65, 1998.

[19] K. G. Wilson. Non-Lagrangian Models of Current Algebra. Phys. Rev., 179(5):1499-1512, Mar 1969.

[20] F. Lehner. Flavour-Changing Neutral Currents at Hadron Colliders, 2005.

[21] A. Bruce et al. Mass of the top quark and induced decay and mixing of neutral $B$ mesons. Phys. Rev. D, 25(7):1989-1992, Apr 1982.

[22] S. Bertolini et al. QCD enhancement of radiative B decays. Phys. Rev. Lett., 59(2):180-182, Jul 1987.

[23] N. G. Deshpande et al. Prediction of $B \rightarrow K \gamma$ as a test of the standard model. Phys. Rev. Lett., 59(2):183-185, Jul 1987.

[24] M. Misiak et al. The first estimate of $\mathrm{B}\left(\bar{B} \rightarrow X_{s} \gamma\right)$ at $\mathrm{O}\left(\alpha_{s}^{2}\right)$. Phys. Rev. Lett., 98:022002, 2007.

[25] A. Limosani (Belle Collaboration). Belle New result on $B \rightarrow X_{s} \gamma$ decay. Results presented in Moriond EW 2008, 2008.

[26] B. Aubert et al. (BaBar Collaboration). Measurement of the $B \rightarrow X_{s} \gamma$ Branching Fraction and Photon Energy Spectrum using the Recoil Method. Phys. Rev D, 77:051103, 2008.

[27] Heavy Flavor Averaging Group and : E. Barberio et al. Averages of b-hadron Properties at the End of 2007, 2008.

[28] A. Ali et al. A Comparative Study of the Decays $B \rightarrow\left(K, K^{*}\right) l^{+} l^{-}$in Standard Model and Supersymmetric Theories. Physical Review D, 61:074024, 2000. 
[29] T. Feldmann and J. Matias. Forward-backward and isospin asymmetry for $B \rightarrow$ $K^{*} l^{+} l^{-}$decay in the standard model and in supersymmetry. JHEP, 0301:074, 2003.

[30] T. Hurth. Present Status of Inclusive Rare B Decays. Reviews of Modern Physics, 75:1159, 2003.

[31] A. Ali et al. Improved model-independent analysis of semileptonic and radiative rare $B$ decays. Phys. Rev. D, 66(3):034002, Aug 2002.

[32] D. Melikhov et al. Rare exclusive semileptonic $b \rightarrow s$ transitions in the standard model. Phys. Rev. D, 57(11):6814-6828, Jun 1998.

[33] K. Abe et al. Observation of the Decay $B \rightarrow K l^{+} l^{-}$. Phys. Rev. Lett., 88(2):021801, Dec 2001.

[34] A. Ishikawa et al. Observation of $B \rightarrow K^{*} \ell^{+} \ell^{-}$. Phys. Rev. Lett., 91(26):261601, Dec 2003.

[35] B. Aubert et al. Evidence for the Rare Decay $B \rightarrow K^{*} \ell^{+} \ell^{-}$and Measurement of the $B \rightarrow K \ell^{+} \ell^{-}$Branching Fraction. Phys. Rev. Lett., 91(22):221802, Nov 2003.

[36] I. Adachi et al. (Belle Collaboration). Measurement of the Differential Branching Fraction and Forward-Backward Asymmetry for $B \rightarrow K^{(*)} \ell^{+} \ell^{-}, 2008$.

[37] B. Aubert et al. (BaBar Collaboration). Measurements of the rare decays $B^{ \pm} \rightarrow$ $K \ell^{+} \ell^{-}$and $B_{d} \rightarrow K^{*} \ell^{+} \ell^{-}$. hep-ex/0507005, 2005.

[38] A. Ishikawa et al. (Belle Collaboration). Measurement of Forward-Backward Asymmetry and Wilson Coefficients in $B \rightarrow K^{*} \ell^{+} \ell^{-}, 2005$.

[39] Fermi national accelerator laboratory http://www.fnal.gov/.

[40] S. Abachi et al. (DØ Collaboration). The DØ detector. NIM, A338:185, 1994.

[41] F. Abe et al. (CDF Collaboration). The CDF detector: an overview. Nucl. Instr. Meth., A271:387-403, 1988.

[42] F. Abe et al. Observation of Top Quark Production in $p \bar{p}$ Collisions with the Collider Detector at Fermilab. Phys. Rev. Lett., 74(14):2626-2631, 1995.

[43] S. Abachi et al. Observation of the Top Quark. Phys. Rev. Lett., 74(14):2632-2637, Apr 1995. 
[44] The Run II handbook

http://www-bd. fnal.gov/runII/index.html.

[45] H.T. Diehl T. LeCompte. The CDF and DØ upgrades for Run II (2000) http://www-d0.fnal.gov/ diehl/Public/annrev/cdfdo.ps.

[46] S. D. Holmes. Design criteria and performance goals for the Fermilab Main Injector. Part. Accel., 58:39-51, 1997.

[47] S. Abachi et al. (DØ Collaboration). The DØ Upgrade: The Detector and its Physics. Technical report, FERMILAB-PUB-96/357-E.

[48] V. M. Abazov et al. (DØ Collaboration). The Upgraded DØ detector. submited to NIM, Fermilab-Pub-05-341-E, physics/0507191, 2005.

[49] E. Kajfasz et al. The DØ silicon microstrip tracker for Run IIa. Nucl. Instrum. Meth., A511:16-19, 2003.

[50] R. C. Ruchti. The Use of Scintillating Fibers for Charged-Particle Tracking. Annual Review of Nuclear and Particle Science, 46:281-319, 1996.

[51] J. Brzenziak et al. Conceptual design of a 2-Tesla superconducting solenoid for the Fermilab D $\varnothing$ detector upgrade. Technical report, FERMILAB-TM-1886, 1994.

[52] DØ Collaboration. Fermilab-pub-02-327-e. Technical report.

[53] DØ Collaboration. DØ Layer 0 Conceptual Design Report. October 2003.

[54] A. Nomerotski. DØ silicon tracker for run iib at the tevatron. Nucl. Instrum. Meth., A518:295-296, 2004.

[55] L. Ancu et al. Analyzing Layer 0 SiDet Cosmic Test Data. DØ-Note-5238, August 2006.

[56] M. Weber. A new inner layer silicon micro-strip detector for DØ. Nucl. Instrum. Meth., A599:182-184, 2006.

[57] D. Tsybychev. Status and performance if the new innermost layer of the silicon detetor at DØ. Nucl. Instrum. Meth., A582:701-704, 2007.

[58] M. Adams et al. Design Report for the Cental Preshower Detector for the D $\varnothing$ Upgrade. DØ-Note-3014, January 1996.

[59] A. Gordeev et al. Design Report for the Forward Preshower Detector for the D $\varnothing$ Upgrade. DØ-Note-3445, May 1998. 
[60] L. Groers. DØ Calorimeter Upgrades for Tevatron Run II. DØ-Note-4240, October 2000. Proceedings for the IXth International Conference on Calorimetry in Particle Physics, Annecy, France, Oct 9-14, 2000.

[61] T. Diehl et al. Technical Design of the Cental Muon System. DØ-Note-3365, March 1998.

[62] G. Alexeev et al. Technical Design for the DØ Forward Muon Tracking Detector Based on Mini-Drift Tubes. DØ-Note-3366, December 1997.

[63] C. Miao et al. The DØ Run II luminosity monitor. Nucl. Phys. Proc. Suppl., 78:342-347, 1999.

[64] T. Edwards et al. Determination of the effective inelastic $p \bar{p}$ cross-section for the DØ Run II luminosity measurement. 2004. FERMILAB-TM-2278-E.

[65] R. Schwienhorst (DØ Collaboration). The DØ Run II trigger system. Int. J. Mod. Phys., A20:3796-3798, 2005.

[66] D. Edmunds et al. Technical design report for the level 2 global processor. DØNote-3402, February 1998.

[67] A. Boehnlein et al. Description of the DØ L3 Trigger software components. DØNote-3630, April 1999.

[68] DØ Collaboration. d0reco software, http://www-d0.fnal.gov/run2physics/www/algorithm.htm.

[69] A. Khanov. HTF: histogramming method for finding tracks. the algorithm descpription. DØ-Note-3778, 2000.

[70] G. Borissov. Ordering a Chaos...or technical details of AA tracking www-d0.fnal.gov/global_tracking/talks/20030228/. 2003.

[71] P. V. C. Hough. Machine Analysis of Bubble Chamber Pictures. In Int. Conf. on High Energy Accelerators and Instrumentation. CERN, 1959.

[72] R. Fruhwirth. Application of Kalman filtering to track and vertex fitting. Nucl. Instrum. Meth., A262:444-450, 1987.

[73] H. Greenlee. The DØ Kalman Track Fit. DØ-Note-4303, 2003.

[74] O. Peters. Muon Segment Reconstruction - Linked List Algorithm. DØ-Note-3901, 2001. 
[75] C. Philippe et al. Muon identification certification for p17 data. DØ-Note-5157, 2006.

[76] A. Schwartzman and C. Chris Tully. Primary Vertex Reconstruction by Means of Adaptive Vertex Fitting. DØ-Note-4918, 2005.

[77] J. Abdallah et al. (DELPHI Collaboration). b-tagging in DELPHI at LEP. Eur. Phys. J., C32:185-208, 2004.

[78] R. P. Lippmann. An introduction to computing with neural nets. SIGARCH Comput. Archit. News, 16(1):7-25, 1988.

[79] H.-J. Yang et al. Studies of Stability and Robustness for Artificial Neural Networks and Boosted Decision Trees. NUCL.INSTRUM.AND METH.A, 574:342, 2007.

[80] B. P. Roe et al. Boosted Decision Trees as an Alternative to Artificial Neural Networks for Particle Identification. NUCL.INSTRUM.METH.A, 543:577, 2005.

[81] B. P. Roe et al. Boosted Decision Trees, a powerfull event classifier. Phystat05 Proceedings, 2005.

[82] L. Breinman et al. Classification and Regression trees. Wadsworth International Groupe, Belmont, California, 1984.

[83] Y. Freund and R. Schapire. A short introduction to boosting. J. Japan. Soc. for Artif. Intel., 14(5):771-780, 1999.

[84] A. Hocker et al. TMVA - Toolkit for Multivariate Data Analysis, 2007.

[85] Root homepage, http://root.cern.ch/.

[86] G. Punzi. Sensitivity of searches for new signals and its optimization. ECONF, C030908:MODT002, 2003.

[87] $\mathrm{D} \varnothing$ Collaboration. The $\mathrm{D} \varnothing$ run quality database, http://d0db-prd. fnal.gov/run/runquery.html.

[88] T. Diehl V. M. Abazov and R. McCroskey. Good and Bad Muon Global Runs Early in Run II. DØ-Note 3938, April 2002.

[89] D. Bauer (DØ Collaboration). B Physics and Triggers at D $\varnothing$ : Operational Experience. Nucl. Phys. B Proc. Suppl., 170:288-291, 2007.

[90] J. Tmple et al. Upgrade of Level 1 Muon Trigger Logic for the v13 Trigger List. DØ-Note-4566, 2004. 
[91] DØ Collaboration. Pythia webpage, http://www.thep.lu.se/ torbjorn/pythia.html.

[92] DØ Collaboration. The EvtGen package Homepage, http://www.slac.stanford.edu/ lange/evtgen/.

[93] PEP-II Experiment. PEP-II webpage, http://www.slac.stanford.edu/grp/ad/ADPEPII/ADPEPII.html.

[94] KEKB Experiment. KEKB webpage, http://www-acc.kek.jp/KEKB/.

[95] DØ Collaboration. d0mess webpage, http://www-cluedo.fnal.gov/do_mess/.

[96] DØ Collaboration. d0gstar webpage, http://www-d0.fnal.gov/computing/montecarlo/simulation/.

[97] DØ Collaboration. d0sim webpage, http://www-d0.fnal.gov/computing/montecarlo/simulation/.

[98] J. Abdallah et al. (DELPHI Collaboration). A precise measurement of the $\mathrm{B}^{+}, \mathrm{B}^{0}$ and mean b-hadron lifetime with the DELPHI detector at LEP I. Eur. Phys. J., C33:307-324, 2004.

[99] B. Abbott et al (DØ Collaboration). Search for the Decay $B \rightarrow X_{s} \mu^{+} \mu^{-}$. Phys. Lett. B, 423:419, 1998.

[100] R. Bernhard. A new expected upper limit for the rare decay $B_{s}^{0} \rightarrow \mu^{+} \mu^{-}$using $2 \mathrm{fb}^{-1}$ of Run II data. DØ-Note-5343, 2007.

[101] S. W. Youn et al. Trigger Efficiency Study for Run II at DØ Using Inclusive Single Muon Sample. DØ-Note-5522, 2007.

[102] G. J. Feldman and R. D. Cousins. Unified approach to the classical statistical analysis of small signals. Phys. Rev. D, 57(7):3873-3889, Apr 1998.

[103] J. Conrad et al. Including systematic uncertainties in confidence interval construction for Poisson statistics. Phys. Rev. D, 67(1):012002, Jan 2003.

[104] J. Conrad et al. Coverage of Confidence Intervals for Poisson Statistics in presence of Systematic Uncertainties, 2002. 



\section{Acknowledgments}

On this last page I would like to thank to numerous people, who have helped and guided me during this last years of my $\mathrm{PhD}$ studies. This work would have never been possible without your advice and support.

First of all, I am very grateful to my supervisor, Prof. Dr. Ulrich Straumann for providing me the opportunity to be part of his research group as a $\mathrm{PhD}$ student. Besides beeing a great experience on its own, I had the chance to work for seven month directly at the $\mathrm{D} \varnothing$ experiment at FERMILAB close to Chicago.

I would like to thank Frank Lehner and Ralf Bernhard for the introduction to the D $\varnothing$ experiment and for the support during my studies. Thanks to Frank for proof-reading too.

A special thank goes to Olaf Steinkamp for proof-reading and the possibility to be his lecture assistant.

I am also thankful to all colleagues at Zuerich for their help and interesting discussion over the past years. I am thankful to Angela, Christophe, Dima, Jeroen, Jonathan and Matt for their friendly atmosphere in and outside our office.

And thanks to many more people have worked with me along the way. This work would not have been possible without the work of a lot of people at the DØ experiment, the Tevatron collider and FERMILAB. I would like to thank the "Germans" at DØ for the assimilation of the "Swiss guy". Thanks to Guennadi for his help and answers to my questions.

Finally I want to thank my familiy and my close friends for their support and encouragement during all the good and some bad days.

This work has been supported by the Swiss National Science Foundation. 


\section{CURRICULUM VitaE}

\section{PERSONAL INFORMATION}

Last Name: Wenger

First Name: Andreas

Day of Birth:

\section{$\underline{\text { EDUCATION }}$}

2004-2008 Dissertation in Physics, University of Zürich

Search for Rare Decays $B^{ \pm} \rightarrow K^{ \pm} \mu^{+} \mu^{-}$and

$B_{d}^{0} \rightarrow K^{*} \mu^{+} \mu^{-}$with the $D \emptyset$ Experiment

Supervisor: Prof. Dr. Ulrich Straumann

2004 Physics Diploma at ETH Zürich

2003-2004 Diploma Thesis, ETH Zürich

The effect of the earth magnetic field on electromagnetic showers

Supervisor: Prof. Dr. Felicitas Pauss

1998-2003 Study of Physics, ETH Zürich

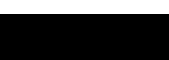

